Aus der Poliklinik für Zahnärztliche Prothetik

(Prof. Dr. med. dent. R. Bürgers)

im Zentrum Zahn-, Mund- und Kieferheilkunde

der Medizinischen Fakultät der Universität Göttingen

\title{
Untersuchung zur Passgenauigkeit CAD/CAM-gefertigter Kronengerüste in Abhängigkeit von den verwendeten Materialien
}

\author{
INAUGURAL-DISSERTATION \\ zur Erlangung des Doktorgrades \\ für Zahnheilkunde \\ der Medizinischen Fakultät der \\ Georg-August-Universität zu Göttingen
}

vorgelegt von

Karin Christine Gronau

aus

Bergisch Gladbach

Göttingen 2015 
Dekan:

I. Berichterstatter:

II. Berichterstatter/in:

III. Berichterstatter/in:
Prof. Dr. rer. nat. H. K. Kroemer PD Dr. med. dent. M. Rödiger Prof. Dr. med. dent. M. Hülsmann Prof. Dr. med. Martin Oppermann

Tag der mündlichen Prüfung: 30.06 .2015 


\section{Inhaltsverzeichnis}

1. Einleitung und Fragestellung ………..................................................... 5

1.1 Grundgedanken und Ziel der vorliegenden Arbeit ................................... 5

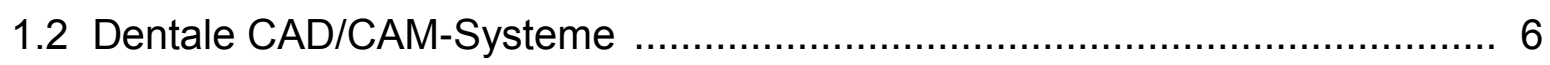

1.2.1 Vorteile der subtraktiven Fertigung mittels CAD/CAM-Systemen gegenüber konventionellen Fertigungstechniken ................................ 6

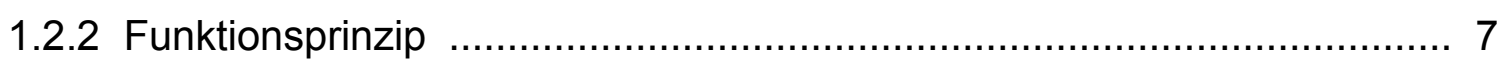

1.2.3 Digitalisierungssystematiken ……………….............................. 7

1.2.4 CAD-Prozess/CAM-Prozess ………………................................ 9

1.2.5 Aktuelle CAD/CAM-Systeme .................................................... 10

1.2.5.1 CEREC (Sirona, Bensheim) ………….................................. 10

1.2.5.2 Lava (3M ESPE AG, Seefeld) ............................................... 12

1.2.5.3 KaVo Everest (KaVo Dental GmbH, Biberach/Riß) ...................... 13

1.2.5.4 KaVo ARCTICA (KaVo Dental GmbH, Biberach/Riß) ..................... 14

1.2.5.5 Cercon smart Ceramics (DeguDent, Hanau) ............................... 16

1.2.5.6 GAMMA (Wissner, Göttingen) .................................................. 18

1.3 Dentale Werkstoffe für die CAD/CAM-basierte Verarbeitung ..................... 21

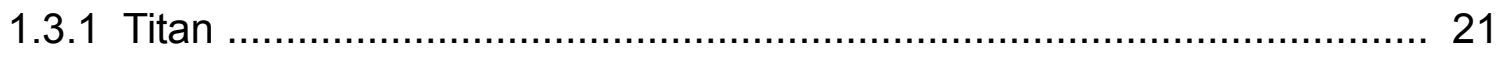

1.3.2 Kobalt-Chrom-Legierungen ……............................................. 23

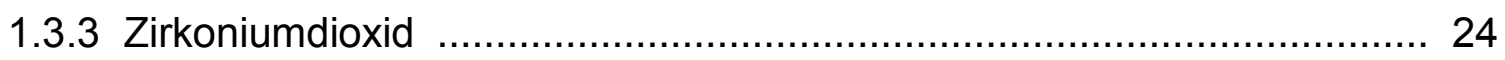

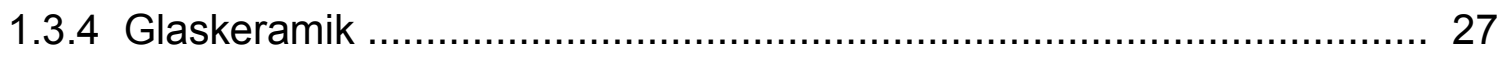

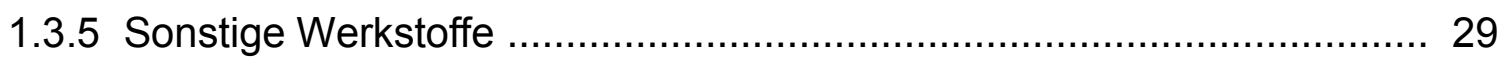

1.4 Relevanz der marginalen Passgenauigkeit ........................................... 30

1.5 Präparationsgrenze ...................................................................... 33

1.5.1 Klinische Bedeutung der Präparationsgrenze ................................... 33

1.5.2 Präparationsformen und -geometrien ............................................ 34

1.5.2.1 Tangentialpräparation ......................................................... 34

1.5.2.2 Hohlkehlpräparation ........................................................ 34

1.5.2.3 Stufenpräparation ........................................................... 35

1.5.2.4 Stufenpräparation mit Abschrägung …………......................... 36 


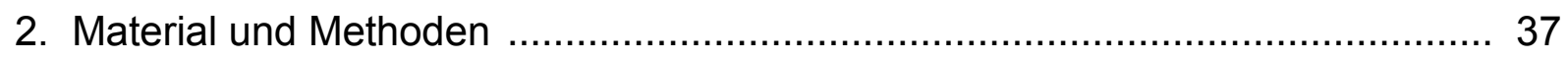

2.1 Herstellung des Masterstumpfs ........................................................... 37

2.2 Herstellung der Meistermodelle (Replika) …….................................... 38

2.3 Herstellung der Kronengerüste ...................................................... 40

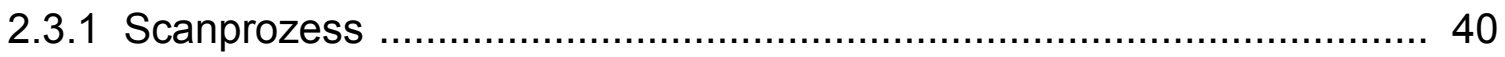

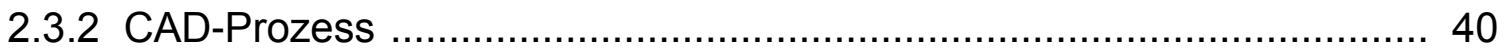

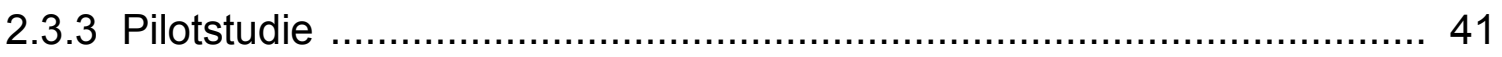

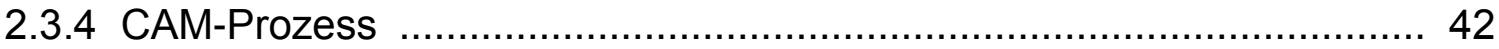

2.4 Unterschiede bei der Herstellung …………................................... 43

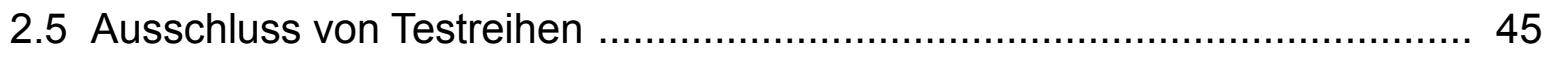

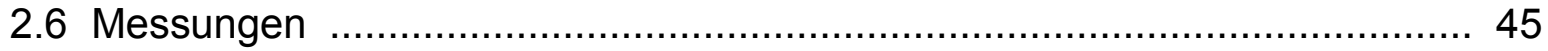

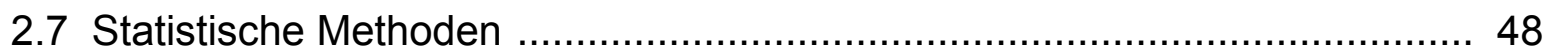

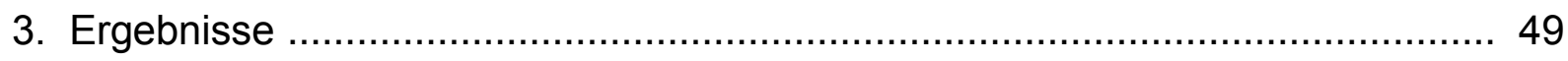

3.1 Statistische Auswertung der Randspaltmessungen ................................. 49

3.2 Marginale Präzision bei der Verwendung von Titan ................................ 50

3.3 Marginale Präzision bei der Verwendung von Kobalt-Chrom ..................... 52

3.4 Marginale Präzision bei der Verwendung von Zirkoniumdioxid .................. 54

3.5 Marginale Präzision bei der Verwendung von Glaskeramik ....................... 56

3.6 Grafische Darstellung der Ergebnisse ................................................. 58

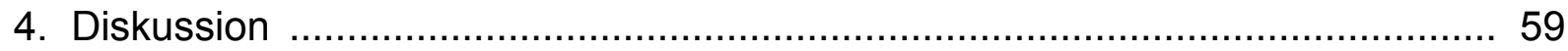

4.1 Beurteilung der Ergebnisse ........................................................ 59

4.2 Vergleich der Ergebnisse der Studie mit anderen Studien ........................ 68

4.3 Schlussfolgerung für die Praxis .......................................................... 71

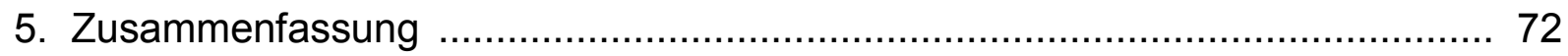

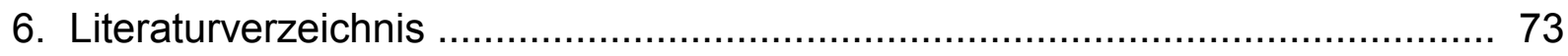




\section{Einleitung und Fragestellung}

\subsection{Grundgedanken und Ziel der vorliegenden Arbeit}

Konventionelle Vollgusskronen werden aufgrund der steigenden Nachfrage nach ästhetischen Versorgungen auch im Seitenzahnbereich immer weiter von metallkeramischen Verblendkronen oder Vollkeramikkronen verdrängt. Dabei stehen nicht nur die Ästhetik, sondern auch die Biokompatibilität und die Funktionalität im Vordergrund. Bevor Keramikverblendungen auf den Markt kamen, wurden Kunststoffverblendungen eingesetzt. Diese konnten an die Zahnfarbe angepasst werden und erfüllten in den ersten Monaten nach der Eingliederung die ästhetischen Ansprüche der Patienten. Durch Wasseraufnahme und Farbveränderungen wurde die Ästhetik nach Untersuchungen von Meyer und Eichner (1980) jedoch schon nach 1,5 Jahren Tragezeit stark eingeschränkt. Die mangelnde Abrasionsfestigkeit des Kunststoffs führte bei Verblendung der Kauflächen außerdem zu Veränderungen der Okklusion bzw. zu einer Verlängerung des Antagonisten (Meyer und Eichner 1980).

Daher wurden Kunststoffverblendungen von Keramikverblendungen abgelöst. Keramikverblendungen haben eine bessere ästhetische Wirkung und Langzeitprognose (Kerschbaum et al. 1997). Durch Rezessionen der Gingiva wird jedoch häufig der Metallrand sichtbar. Dies führt zu einem ungenügenden ästhetischen Ergebnis. Um im Frontzahngebiet auf das metallische Gerüst verzichten zu können, wird stattdessen vollkeramischer Zahnersatz eingesetzt.

Durch neue Technologien wie das Computer-Aided Design/Computer-Aided Manufacturing-Verfahren (CAD/CAM-Verfahren) und die Verfügbarkeit von hochfesten Gerüstkeramiken können Vollkeramikkronen mit ausreichender Festigkeit hergestellt werden. Diese Vollkeramikkronen können auch im stark belasteten Seitenzahnbereich eingesetzt werden (Belli et al. 2011; Rinke und Fischer 2012; Tinschert et al. 1999). Sie führen dort zu ähnlichen klinischen Resultaten, wie sie bei Metallkeramik-Restaurationen erreicht werden (Vigolo und Mutinelli 2012). 
Sowohl die metallkeramischen als auch die vollkeramischen Restaurationen besitzen ausreichende mechanische Eigenschaften. Somit entscheidet vor allem die innere Passgenauigkeit und die Größe des Randspalts über die Qualität der Versorgungen, da ein ungenügender Randschluss zum Beispiel zu Sekundär-Karies führen kann (Karlsson 1986; Rehberg 1971).

Daher ist es das Ziel der vorliegenden Arbeit, die Größen der marginalen Randspalten von CAD/CAM-gefertigten Kronen aus verschiedenen Materialien zu messen und $\mathrm{zu}$ vergleichen. Da bei der Verwendung von unterschiedlichen CAD/CAM-Systemen die Passungen verschieden sind (Kohorst et al. 2009; Vigolo und Fonzi 2008), werden für diese Arbeit alle Materialien mit demselben CAD/CAMSystem der Firma Wissner (Göttingen) gefertigt. Damit soll der Einfluss der CAD/CAM-Systeme auf die marginale Passgenauigkeit ausgeschlossen werden, um isoliert die Auswahl des Werkstoffs als Einflussgröße zu evaluieren.

\subsection{Dentale CAD/CAM-Systeme}

\subsubsection{Vorteile der subtraktiven Fertigung mittels CAD/CAM-Systemen gegenüber konventionellen Fertigungstechniken}

Durch die Einführung der CAD/CAM-Systeme in den zahntechnischen Bereich können erstmals hochfeste Materialien wie Zirkoniumdioxid unter wirtschaftlichen Aspekten adäquat bearbeitet werden.

Somit erweitert sich das Indikationsspektrum, und es können auch mehrgliedrige Brücken aus diesen Keramiken im Seitenzahnbereich eingegliedert werden. Ein weiterer Vorteil der Einführung der CAD/CAM-Systeme ist die Nutzung von Materialien, deren Qualität und Reinheit (homogenes Gefüge) durch standardisierte industrielle Herstellungsprozesse annähernd gleichbleibend sind.

Im Gegensatz dazu weisen konventionelle Vollgusskronen oft Inhomogenitäten auf, da es während des Gießprozesses durch den Volumenverlust des Metalls beim Erstarren zu zahlreichen Poren und Lunkern kommen kann. Zudem fließen dünn auslaufende Ränder häufig nicht vollkommen aus (Jung und Quast 1971). 
Diese Probleme entfallen bei subtraktiven CAD/CAM-gestützten Verfahren, da die Restaurationen aus den industriell hergestellten Materialblöcken gefräst werden.

Durch die Einführung der CAD/CAM-Technologie soll nicht nur eine gleich bleibende Qualität gesichert, sondern es sollen auch die Produktionskosten durch den automatisierten Fräsprozess gesenkt werden.

\subsubsection{Funktionsprinzip}

Die heute üblichen computerunterstützten Fertigungssysteme weisen einen ähnlichen Herstellungsprozess auf. Dieser kann in drei aufeinander folgende Schritte unterteilt werden:

- Digitalisieren (Scannen)

- Computerunterstützte Konstruktion (CAD)

- Computerunterstützte Fertigung (CAM)

Als erstes muss die intraorale Situation digitalisiert werden. Dazu wird die Präparation durch einen Scanner in ein virtuelles Bild übertragen. Der entstandene Rohdatensatz wird dann in den Modelldatensatz umgewandelt und visualisiert. Mit Hilfe einer CAD-Software wird die Restauration konstruiert und der Konstruktionsdatensatz generiert. Im Anschluss daran berechnet die Software die Fräsbahnen zur Fertigung der Restauration. Zum Schluss wird der reale Zahnersatz aus einem Rohling computergesteuert gefräst bzw. geschliffen.

\subsubsection{Digitalisierungssystematiken}

Zur Digitalisierung des präparierten Stumpfs kann das intraorale oder das extraorale Verfahren angewandt werden.

Bei den sogenannten Chairside-Systemen kann die optische Abformung intraoral durchgeführt werden und die virtuelle Modellherstellung am Behandlungsstuhl stattfinden. In derselben Behandlungssitzung wird die computergestützte Konstruktion des Zahnersatzes durchgeführt und die Fertigung sowie die 
Eingliederung der künstlichen Zähne können sofort erfolgen. Mit dem ChairsideSystem können auf diese Weise Einzelzahnrestaurationen bis hin zu provisorischen viergliedrigen Brücken hergestellt werden (Reich et al. 2012). Lange unbezahnte Kieferabschnitte können jedoch noch nicht ausreichend präzise abgeformt werden.

Der Vorteil der intraoralen optischen Abformung ist das Überspringen der konventionellen Arbeitsschritte. Abformung, Gipsmodellherstellung und das Einscannen des Gipsmodells - mit ihren entsprechenden inhärenten Fehlerquellen entfallen. Die Präparation kann am Computer kontrolliert und gegebenenfalls im Patientenmund selektiv korrigiert werden. Zusatztermine zur erneuten Abdrucknahme oder Nachpräparation entfallen (Müller und Trützschler 2012).

Ein Nachteil einiger Systeme ist, dass vor der Abdrucknahme auf den abzuformenden Bereich ein Puder aufgetragen werden muss. Um zu verhindern, dass es durch das hygroskopische Puder zu Fehlmessungen an der Präparationsgrenze kommt, muss der Bereich ausreichend trocken gehalten werden. Eine beschädigte Puderschicht kann zu Störungen in dem vom Computer simulierten Modell führen. Bei einer vorsichtigen Nachpuderung dürfen keine Puderanhäufungen auftreten, da sie wiederum zu Fehlmessungen führen würden. In der Studie von An et al. (2014) führte jedoch auch eine puderfreie Chairside-Variante zu größeren Randspalten als das getestete extraorale Verfahren.

Die Präparationsgrenze muss bei intraoralen optischen Abformungen im sichtbaren Bereich liegen, da ein Verdrängen der Gingiva, wie es mit konventionellen Abdruckverfahren teilweise möglich ist, mit optischen Systemen nicht realisierbar ist (Reich et al. 2012).

Bei den extraoralen Labside-Systemen ist es nötig, für die Erstellung eines Gipsmodells einen konventionellen Abdruck zu nehmen. Zur Digitalisierung des Gipsmodells kann das mechanische (taktile) oder das berührungsfrei-optische Verfahren angewendet werden. Bei dem mechanischen Verfahren wird ein Taster über die Modelloberfläche geführt. Dabei zeigen manuell geführte Tastsysteme hohe Messungenauigkeiten (Luthardt et al. 2001). Bei maschinellen Tastsystemen entscheiden unter anderem der Anpressdruck, die Größe des Tasters und die Digitalisierungsgeschwindigkeit über die exakte Registrierung des Objekts. Zu großer Anpressdruck und zu kleine Taster können das Gipsmodell beschädigen, während zu große Taster feine Unebenheiten auf der Oberfläche nicht wahrnehmen 
können (Luthardt et al. 2009). Auch der Winkel zwischen Taster und Objekt ist entscheidend für die Genauigkeit. Je kleiner dieser Winkel ist, desto geringer ist die Messgenauigkeit (Hewlett et al. 1992).

Die optischen Verfahren arbeiten berührungslos. Sie können, wie oben beschrieben, auch intraoral angewandt werden. Begrenzt werden die intraoralen Systeme durch das geringe Platzangebot und die hohe Luftfeuchtigkeit. Die extraoralen Systeme arbeiten in der Mehrzahl mit einer Projektor- und einer Detektoreinheit. Diese Einheiten sind in einem bekannten Verhältnis zueinander angeordnet.

Die Projektoreinheit sendet Laserstrahlen oder Weißlicht flächenhaft, punkt- oder linienförmig aus. Die Strahlen werden von der Oberfläche des zu digitalisierenden Objekts reflektiert. Die Detektoreinheit nimmt die reflektierten Strahlen wahr und es kann eine digitale Punktewolke errechnet werden. Diese Punktewolke wird in den Modelldatensatz umgewandelt und am Monitor als dreidimensionales Abbild gezeigt.

\subsubsection{CAD-Prozess/CAM-Prozess}

Bevor die computerunterstützte Fertigung erfolgen kann, muss zunächst aus den individuellen Patientenrohdaten ein Konstruktionsdatensatz erstellt werden.

Möchte man die Restauration nicht am Computer entwerfen, kann durch ein elektronisches Kopierschleifsystem die zuvor konventionell erstellte Wachsmodellation in einen Konstruktionsdatensatz umgewandelt werden. Die meisten Systeme bieten jedoch eine computerunterstützte Konstruktion der dentalen Restauration an. Obwohl die Programme die Päparationsgrenze des eingescannten Stumpfes oft selbstständig finden, sind eine Kontrolle und eine eventuelle Korrektur durch den Anwender erforderlich. Die Schichtstärke des Gerüsts und die Größe des Zementspalts können individuell eingestellt werden.

Bei monolithischen vollanatomischen Restaurationen reichen diese Schritte nicht aus. Es muss eine Kaufläche gestaltet werden, die auf die Okklusion des Patienten abgestimmt ist. Wurde vor der Präparation die klinische Situation digitalisiert, kann die individuelle Kaufläche rekonstruiert werden. Ansonsten ist es auch möglich, eine konventionell gefertigte Wachsmodellation zu digitalisieren oder auf Kauflächen aus Zahn-Bibliotheken zurückzugreifen. 
Der fertige Konstruktionsdatensatz wird in den Fertigungsdatensatz umgerechnet. Bei subtraktiven CAD/CAM-Systemen enthält der Fertigungsdatensatz die Werkzeugbahnen, die das System ablaufen muss, um die Restauration zu erstellen. Für jedes Material gibt es geeignete Werkzeuge, die durch die Maschine ausgewählt werden. Bei der Verwendung von Werkstoffen, die nach der Bearbeitung eine Dimensionsänderung erfahren (zum Beispiel durch Sinterung), muss diese Dimensionsänderung im Fräsprozess berücksichtigt werden.

Der Fräsprozess umfasst gewöhnlich mindestens zwei Bearbeitungsschritte. Zuerst wird die grobe Form durch Schruppen bzw. die Grobbearbeitung vorgegeben. Danach erfolgt mit einem anderen Werkzeug die Feinbearbeitung durch das Schlichten. Bei dem Schruppen soll ein möglichst großer Substanzabtrag in kurzer Zeit erreicht werden (großes Zeitspanvolumen), um mit der Feinbearbeitung zügig beginnen zu können. Das Schlichten soll eine hohe Maß-, Form- und Oberflächengüte erzielen, muss dabei jedoch auch effizient und wirtschaftlich sein.

\subsubsection{Aktuelle CAD/CAM-Systeme}

\subsubsection{CEREC (Sirona, Bensheim)}

Das CEREC CAD/CAM-System wurde im klinischen Bereich ab 1987 eingesetzt. Mittlerweile ist CEREC das am häufigsten genutzte System (Reiss 2006). Es ist auf die Chairside-Fertigung spezialisiert. Die Intraoralkamera von CEREC (CEREC Bluecam) löst automatisch in einem geeigneten Aufnahmemoment aus. Dazu wird die Kamera stückweise über den abzubildenden Bereich geführt. Die Verwacklungsdetektion sorgt dafür, dass nur dann ein Bild aufgenommen wird, wenn die Kamera ruhig steht. So ist die Abdrucknahme eines gesamten Quadranten in weniger als einer Minute möglich. Dabei erreicht die Bluecam bei Einzelzahnaufnahmen eine Präzision von $19 \mu \mathrm{m}$, bei Aufnahmen des gesamten Quadranten ist eine Genauigkeit von 35 m möglich (Mehl et al. 2009). Durch die kleine Bauform der Kamera ist es möglich, auch den hinteren Seitenzahnbereich abzuformen. Für die Ermittlung der Okklusion zur Erstellung von vollanatomischen Kronen wird der individuelle Biss durch eine bukkale Aufnahme festgehalten. 
Die CEREC-Software ermittelt selbstständig geeignete Aufnahmen und generiert aus innen ein dreidimensionales, virtuelles Modell.

Die virtuelle Konstruktion der Restauration wird durch die CEREC-Software unterstützt. Die Software hilft bei der Markierung der Präparationsgrenze. Durch die schwenkbare 3-D Ansicht kann die Präparation von allen Seiten betrachtet werden. Die Modellation der Restauration erfolgt direkt an dem virtuellen Zahn, sodass der Zahnersatz intuitiv entworfen werden kann. Mit der CEREC-Software ist es außerdem möglich, mehrere Restaurationen parallel zu bearbeiten. Zum Beispiel können Veneers, Kronen oder Brücken gleichzeitig und aufeinander abgestimmt konstruiert werden.

Soll eine monolithische Krone angefertigt werden, kann das CEREC BiogenerikVerfahren eingesetzt werden. Es leitet die individuelle Morphologie des zu ersetzenden Zahnes von einem eingescannten Referenzzahn ab. Dadurch entfällt das Suchen in Zahnbibliotheken nach passenden Morphologien. Obwohl auch die mit Biogenerik erstellten Kronen noch am Computer kontrolliert und korrigiert werden müssen, ist die Biogenerik-Software in der anatomischen Kauflächengestaltung der Standard-CEREC-Software überlegen (Reiss 2007).

Ist die Modellation abgeschlossen, werden die Daten an die CEREC-Praxismaschine übermittelt. Diese schleift die Kronen in fünf bis zehn Minuten. Sie nutzt dabei ein Zwillings-Schleifkonzept, mit dem der Rohling von zwei Diamanten gleichzeitig bearbeitet werden kann.

Alternativ können die Daten auch über das Sirona Connect Portal zu einem zahntechnischen Labor geschickt werden. Ein notwendiges Provisorium kann dann in der gleichen Sitzung mit CEREC in der Praxis hergestellt und eingesetzt werden. Dieses wird getragen, bis die definitive Versorgung fertig ist.

Weiterhin können Brücken mit bis zu 12 Gliedern, individuelle Abutments, Teleskope u.a. hergestellt werden. Vollanatomische Kronen können zum Beispiel im CERECSinterofen inFire HTC speed (Sirona, Bensheim) innerhalb von zwei Stunden gesintert werden. 


\subsubsection{Lava (3M ESPE AG, Seefeld)}

Mit dem Lava Chairside Oral Scanner (C.O.S.) hat auch die 3M ESPE AG einen Intraoralscanner entwickelt. Die so genannte 3D-in-Motion-Technologie nimmt die Daten als Videosequenz auf. In einer Sekunde werden auf diese Weise ungefähr 20 3D-Datensätze erstellt. Es soll damit auch bei hoher Geschwindigkeit gewährleistet werden, die Oberflächen gleichmäßig zu scannen. Für den Scan eines gesamten Kiefers braucht der Lava C.O.S. ca. zwei Minuten. In dieser Zeit entstehen 2400 3DBilder. Auf dem Touchscreen zeigt die Software von Lava die Entfernung der Präparation zum antagonistischen Zahn, sodass bei Bedarf noch einmal intraoral nachpräpariert werden kann (Lava ${ }^{\mathrm{TM}}$ Chairside Oral Scanner 2011).

Durch die zweite Version des Lava Scan ST können wie gewohnt Gipsmodelle eingescannt werden. Dabei kann der komplette Kieferbogen oder nur der präparierte Zahn übertragen werden. Für den Scan mehrerer Einzelstümpfe wurde der Lava Multi-Die-Träger entwickelt. Bis zu 10 Einzelstümpfe können gleichzeitig in 2 Minuten und 20 Sekunden gescannt werden, sodass pro präpariertem Zahn eine Scannzeit von 14 Sekunden entsteht. Außerdem ist es möglich, den Gegenkiefer gesondert oder in Okklusion zu digitalisieren, um die okklusalen Kontakte der Restauration gestalten zu können. Als Konstruktionshilfe und für das automatische Kopierfräsen können Wax-ups digitalisiert werden (Lava ${ }^{\mathrm{TM}}$ Scan ST Design System 2011).

Die Lava Design Software kann innerhalb einer Minute die Präparationsgrenze einzeichnen und eine vollanatomische Krone entwerfen. Diese kann individualisiert oder durch eine Konstruktion aus einer Bibliothek ersetzt werden.

Soll anstelle einer vollanatomischen Krone ein Gerüst hergestellt werden, wird dies durch die Lava Design Software ermöglicht. Statt der Lava-Keramik können durch ein optionales Software-Modul auch Metallrestaurationen virtuell konstruiert werden.

Die Daten der digitalen Modellationen werden über die Lava Connect-Schnittstelle an ein Lava-Fräszentrum übertragen. 


\subsubsection{KaVo Everest (KaVo Dental GmbH, Biberach/Riß)}

Das KaVo Everest-System bietet zur Digitalisierung der Modelle zwei Scanner an, den Everest Scan pro und den Everest Scan II. Der Everest Scan pro benötigt für die Messung eines Stumpfs drei Minuten. In diesen drei Minuten wird der Stumpf aus 15 unterschiedlichen Positionen von einer CCD-Kamera erfasst. Um unter sich gehende Bereiche aufnehmen zu können, dreht sich der Objektträger um seine horizontale und vertikale Achse. Ebenso wie der Everest Scan pro arbeitet der Everest Scan II mit einem Streifenlichtmessverfahren. Er benötigt für einen Stumpf mit 95 Sekunden ungefähr die Hälfte der Zeit des Everest Scan pro. Wurden komplizierte Kavitäten in dieser Zeit nicht vollständig erfasst, können zusätzliche Aufnahmen gemacht werden. Neben den beiden Scannern besteht das Everest-System außerdem aus folgenden Komponenten:

- CAD-Software (Energy CAD)

- Schleifeinheit (Everest Engine)

- Materialien (Everest Elements)

- Sintereinheit (Everest Therm)

Mit dem Structure-Softwaremodul (Energy CAD-Software) ist das Fertigen von Basisrestaurationen bis hin zu Brücken möglich. Mit weiteren Software-Programmen können Implantataufbauten konstruiert und vollanatomische Kronen aus einer Zahndatenbank ausgewählt werden. Innerhalb der Software wird durch intuitiv einsetzbare Tools wie das Wachsmesser der konventionelle Arbeitsablauf simuliert. Das 3D-Modell kann gedreht werden, um es von allen Seiten betrachten und bearbeiten zu können. Die Abmessungen der Restaurationen lassen sich ändern, allerdings sind für eine höhere Anwendersicherheit Mindeststärken der Restaurationen hinterlegt (Wischropp 2008).

Die Everest Elements-Materialien sind mit einer Codierung versehen, die auf einem Chip gespeichert ist. Dieser Chip enthält zum Beispiel Informationen zum Material, zur Herkunft und bei Keramiken zur Sinterschwindung. Diese Informationen werden von der Everest CAM-Software erkannt und verarbeitet. Die Everest Elements werden als Blank oder als Ronde angeboten. 
Aus einer Ronde können bis zu 25 Einheiten hergestellt werden. Das EverestSystem merkt sich die Restkapazität der Ronden, sodass eine gute Ausnutzung der Ronden realisierbar ist.

Die Blanks sind in eine Kunststoffform eingebettet, durch die hindurch als erstes die Innenseite der Restauration ausgefräst wird. Ist die Innenkontur beendet, wird der Rohling abgespült, getrocknet und kurz erwärmt. Anschließend wird manuell neuer Kunststoff eingegossen, der ca. sechs Minuten aushärtet. Das Autostart Programm startet nach dieser Zeit die Bearbeitung der Außenseite. Nach Abschluss des Fräsvorgangs wird der Kunststoff im Ofen bei $1000^{\circ} \mathrm{C}$ erhitzt und der Zahnersatz kann entnommen werden. Bei Zirkoniumdioxid wird statt Kunststoff schnellhärtendes Wachs verwendet. Durch den Kunststoff bzw. den Wachs kann auf Haltestifte an der Restauration verzichtet werden. So werden anfallende Nacharbeiten reduziert (Wischropp 2008).

Durch unterschiedliche Spannbrücken für die Schleifeinheit ist es möglich, verschiedene Materialien einzulegen. So können mit der Spannbrücke für zwei Ronden ohne Materialwechsel bis zu 50 Einheiten hergestellt werden.

Das Sintern der Keramik ist im Everest Therm möglich. Er kann durch das Bedienelement oder einen Computer gesteuert werden. Durch die Brennraumerhitzung entsteht eine gleichmäßige Wärmeverteilung. Aufgrund der Hochleistungsisolierung verbleibt die Wärme im Inneren des Geräts und wird nicht nach draußen abgegeben.

\subsubsection{KaVo ARCTICA (KaVo Dental GmbH, Biberach/Riß)}

Die KaVo Dental GmbH produziert neben dem KaVo Everest CAD/CAM-System auch das ARCTICA Dental CAD/CAM-System. Im Mittelpunkt des ARCTICA-Systems steht die ARCTICA-Engine. Diese dentale Schleif-/Fräsmaschine wird seit 2012 angeboten. 
Das komplette KaVo ARCTICA CAD/CAM-System besteht aus folgenden Elementen:

- Scaneinheit (KaVo ARCTICA Scan)

- CAD-Software (KaVo multiCAD)

- Schleif-/Fräsmaschine (KaVo ARCTICA Engine)

- Materialien (KaVo ARCTICA Elements)

Der CAD/CAM-Prozess beginnt mit der Digitalisierung des Gipsmodells mit dem KaVo ARCTICA Scan. Dieser Scanner arbeitet mit Streifenlichtprojektion und braucht für einen Einzelstumpf ca. eine Minute Scanzeit. Die Daten werden an die KaVo multiCAD-Software übermittelt. Sie können jedoch auch von CAD-Software anderer Hersteller verarbeitet werden. Bei der multiCAD-Software führt der Schritt-für-SchrittAssistent durch die Gestaltung. So wird sichergestellt, dass kein Arbeitsschritt übersprungen wird. Außerdem hilft der Assistent bei der Erkennung der Präparationsgrenze, bei der Konstruktion der Restaurationsinnenseite, bei der Platzierung der Bibliothekszähne und bei dem Einfügen von Verbindern bei Brücken. Ist die Gestaltung der Restauration abgeschlossen, können die Daten über das KaVo Everest Portal an die ARCTICA Engine übertragen werden. Über das Portal kann auch ein externes Labor beauftragt werden die Fertigung zu übernehmen.

Die KaVo ARCTICA Engine zeigt für die jeweilige Konstruktion das geeignete Werkzeug an. Das ausgewählte Werkzeug wird vor der Verarbeitung mit einem Laser auf Bruch oder Fehlpositionierung überprüft. Der Abnutzungsgrad der Werkzeuge wird durch eine Ampel-Farbkodierung angezeigt (Ewertz 2013). Auf diese Weise werden vor jedem Verarbeitungsvorgang die Werkzeuge automatisch überprüft, um zu verhindern, dass defekte oder verschlissene Werkzeuge verwendet werden. Durch das 5-Achssystem können auch komplizierte unter sich gehende Bereiche gefräst werden.

Die KaVo ARCTICA Engine wird in zwei Varianten angeboten. Bei der ersten Variante (offene Maschine) kann Material von Fremdanbietern verarbeitet werden.

Die zweite Variante (geschlossene Maschine) nutzt im Gegensatz dazu ausschließlich KaVo ARCTICA Elements und VITABLOCS (VITA Zahnfabrik, Bad Säckingen) als Rohlinge. 
Die ARCTICA Elements bieten eine umfangreiche Materialauswahl. So können Restaurationen aus Titan, Zirkoniumdioxid, Glaskeramik, Wachs und Kunststoff erstellt werden.

\subsubsection{Cercon smart Ceramics (DeguDent, Hanau)}

Das Cercon-System ist aus dem Züricher DCM-System entstanden. Es besteht aus folgenden Komponenten:

- Scanner (Cercon eye)

- CAD-Software (Cercon art)

- spezielle Computer Maus (Cercon move)

- Schleifeinheiten (Cercon brain und Cercon brain expert)

- Absaugeinheit (Cercon clean)

- Sinteröfen (Cercon heat und Cercon heat plus)

- Materialien, wie zum Beispiel Cercon base, Cercon ht und Cercon ceram kiss

Bevor das Modell in den Cercon eye-Scanner gestellt werden kann, wird die Modellsituation mit Hilfe einer Schablone überprüft. So ist es möglich die richtige Höhe einzustellen. Durch die Schablone kann in diesem Moment die richtige Größe des Cercon base-Materialrohlings ermittelt werden. Vor dem Scanvorgang muss mit der Cercon art-Software die Restaurationsart festgelegt werden (CAD-Krone oder CAD-Brücke). Anschließend fragt das Programm, ob es sich bei dem Modell um einen Ober- oder Unterkiefer handelt und auf welchen Zähnen die Restauration verankert werden soll. Danach wird ausgewählt, aus welchem Material der Zahnersatz gefertigt werden soll. Da die Stümpfe einzeln gescannt werden müssen, werden die übrigen Teile aus dem Modell entfernt. Auf diese Weise bleibt nur der Stumpf übrig, der als erstes digitalisiert werden soll. Um den Stumpf zu scannen, wird er auf dem Scannteller so positioniert, dass das Fadenkreuz mittig auf dem Stumpf liegt. Ist dieses Segment fertig gescannt, gibt das Programm an, welcher Teil danach digitalisiert werden soll. Bei Kronen kann es ein benachbarter Zahn und bei Brücken ein weiterer Brückenanker, ein Gingivaelement oder ein Nachbarzahn sein. 
Sind alle Elemente einzeln gescannt worden, rechnet der Computer ihre Stellung zueinander aus. Dies ist durch die drei Referenzmarken auf dem Modelltisch möglich. Das Ergebnis wird als Punktewolke abgebildet. Diese kann von basal gekürzt werden, um die Datenmenge zu mindern. Um die Arbeit am Computer zu vereinfachen, hat das Unternehmen eine Computer Maus (Cercon move) entwickelt, mit der man die Stellung des Modells intuitiv verändern kann. So wird das Modell bewegt, gekippt, rotiert und stufenlos vergrößert (von Schroeter et al. 2004). Nachdem die digitalen Daten von dem System berechnet wurden, stellt es automatisch die Präparationsgrenze dar, die manuell korrigiert werden kann. Danach schlägt das Programm alle Elemente der Restauration vor. Bei einer Brücke sind dies die Kappen, die Verbinder und die Brückenzwischenglieder. Dieser Vorschlag kann beliebig verändert werden, allerdings warnt das Programm bei Verbindern, deren Querschnittsfläche das Minimum von $9 \mathrm{~mm}^{2}$ unterschreitet. Ist die Konstruktion der Restauration beendet, können die Daten zur laborinternen Schleifeinheit (Cercon brain) oder zur zentralen Fertigung (Compartis) übermittelt werden.

Während bei dem Schleifen das grobe Schruppfräsen mäanderförmig verläuft, werden Feinheiten wie die Innenflächen der Objekte zirkulär bearbeitet. Zum Abschluss des Fräsvorgangs wird die Haltemembran der Restauration perforiert, damit der Zahnersatz später einfacher entnommen werden kann.

Die vorgesinterten Keramikrohlinge werden um den Betrag der Sinterschrumpfung vergrößert ausgefräst, damit sie nach dem Sintern nicht zu klein werden.

Das Sintern geschieht mit den Cercon heat-Systemen. Diese weisen laut Hersteller eine Maximaltemperatur von $1350^{\circ} \mathrm{C}$ auf. Im Cercon heat-Sinterofen finden bis zu 30 Einheiten Platz, die ungefähr sechs Stunden gesintert werden.

Das Zirkoniumdioxid von Cercon hat eine gute Langzeitstabilität und Erfolg auch im Seitenzahnbereich (Rödiger et al. 2010). Cercon base und Cercon ht sind die beiden Zirkoniumdioxidvarianten, die DeguDent anbietet. Cercon base-Rohlinge sind für den Einsatz in der Schleifeinheit von DeguDent (Cercon brain) ausgerichtet und besitzen entweder eine zylindrische Gestalt oder sind scheibenförmig. Die zylindrischen Rohlinge werden in verschiedenen Größen angeboten, sodass Gerüste mit unterschiedlichen anatomischen Längen bis $47 \mathrm{~mm}$ hergestellt werden können. 
Die Cercon base disk-Rohlinge werden in vier verschiedenen Höhen produziert. Durch die Möglichkeit, die Disk-Rohlinge mehrfach zu verwenden, kann gegenüber den zylindrisch geformten Rohlingen wirtschaftlicher gearbeitet werden.

Cercon ht ist ein transluzenteres Zirkoniumdioxid. Da es nach Herstellerangaben die gleiche Langzeitstabilität und Biegefestigkeit wie Cercon base besitzt, kann es für vollanatomische Restaurationen eingesetzt werden.

Außer den beiden Zirkoniumdioxidarten bietet DeguDent Rohlinge aus Kunststoff und Wachs an. Restaurationen aus Titan und Kobalt-Chrom können vom Fertigungszentrum Compartis produziert werden.

\subsubsection{GAMMA (Wissner, Göttingen)}

Die Firma Wissner $\mathrm{GmbH}$ wurde 1986 gegründet und hat sich zu einem mittelständischen Maschinenbauunternehmen entwickelt. Nachdem Rolf Wissner durch den Kontakt zu Prof. Dr. François Duret, dem Begründer der dentalen CAD/CAM-Technologie, am Bau dessen erster Fräsmaschine mitgearbeitet hatte (High Speed Cutting 2009), produzierte er unter anderem dentale Fertigungseinheiten für große dentale CAD/CAM-Fertigungszentren in Deutschland.

Die Wissner $\mathrm{GmbH}$ produziert drei dentale Maschinenvarianten: GAMMA 202, GAMMA 303 und GAMMA 605. Die GAMMA 202 (siehe Abb. 1) ist eine 5-AchsMaschine und die kleinste der drei Systeme. Sie eignet sich vor allem für zahntechnische Labore, die ca. 40 Restaurationen pro Tag anfertigen. Dieses System soll im Rahmen dieser Studie Verwendung finden.
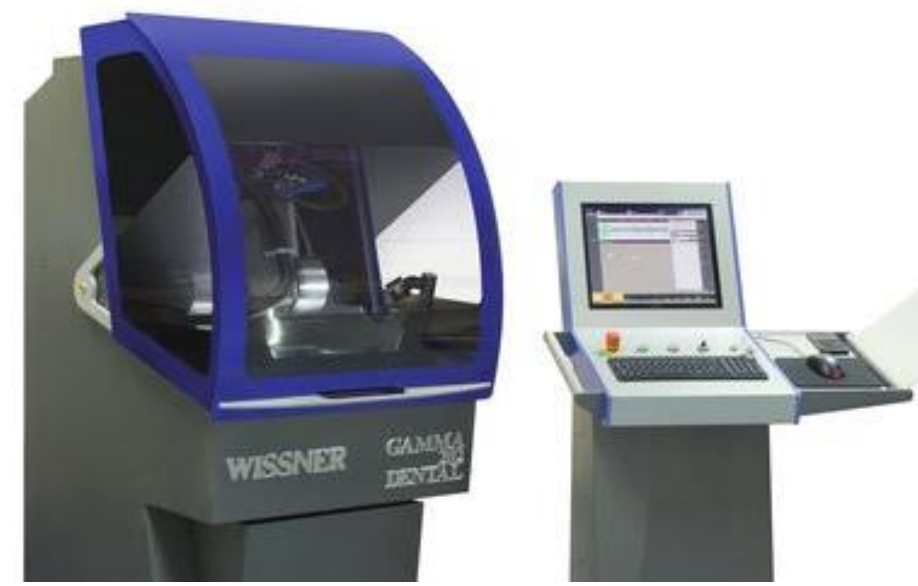

Abb. 1: GAMMA 202 (Quelle: http://www.wissner-gmbh.de/printmedien.htm), mit freundlicher Genehmigung der Wissner GmbH 
Die 4-Achs-Maschine GAMMA 303 und die 5-Achs-Maschine GAMMA 605 kommen für Fräszentren oder größere Labore in Frage (Rißmann 2009). Alle GAMMAFertigungseinheiten haben offene Schnittstellen und können so mit CAD/CAMBestandteilen anderer Hersteller verbunden werden.

Da die Maschinen eine Vielfalt von Materialien wie Titan, Kobalt-Chrom, Kunststoff, Wachs, Zirkoniumdioxid und Glaskeramik bearbeiten können, werden verschiedene Werkzeuge benötigt.

Die Maschinen können mit einem automatischen Werkzeugwechsler (siehe Abb. 2) ausgestattet werden, der je nach Maschine 10 bis 30 Werkzeuge aufnehmen kann. Im Wechsler können auch Ersatzwerkzeuge untergebracht werden, die eingesetzt werden können, sollte ein Werkzeug abbrechen oder sich abnutzen. Die geeigneten Werkzeuge werden ausgewählt und durch ein Messsystem automatisch auf Abnutzung überprüft.

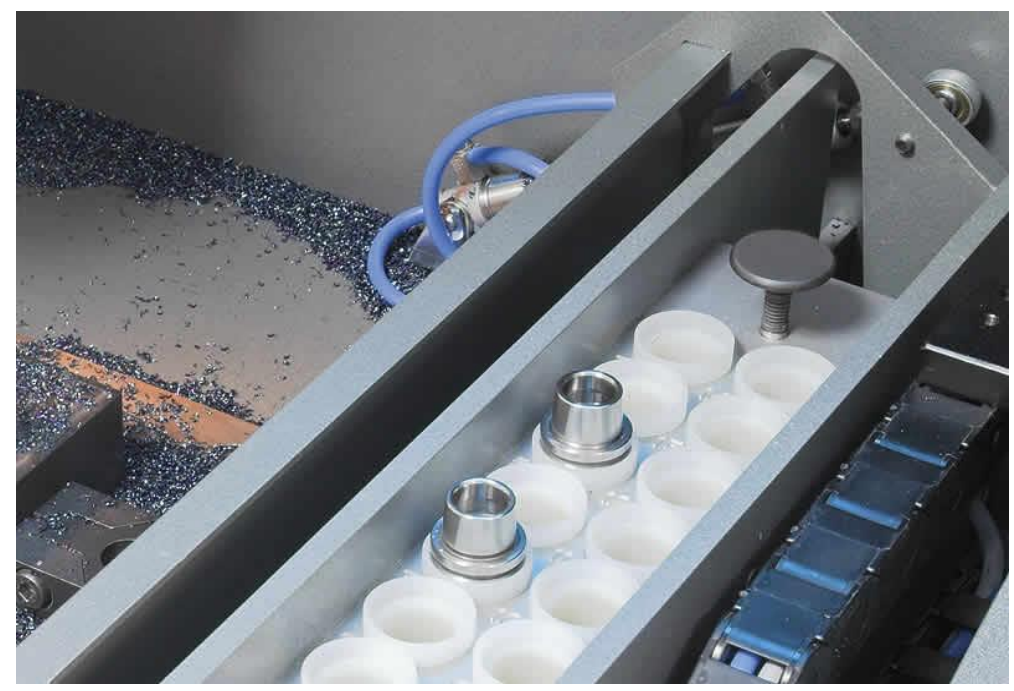

Abb. 2: Werkzeugwechsler (http://www.wissner-gmbh.de/printmedien.htm), mit freundlicher Genehmigung der Wissner GmbH

Im nachrüstbaren Werkstückwechsler (Loader) haben 30 Rohlinge Platz (siehe Abb. 3).

Durch das automatische Wechseln der Werkzeuge und der Rohlinge ist eine unbemannte Fertigung zum Beispiel über das Wochenende möglich. 


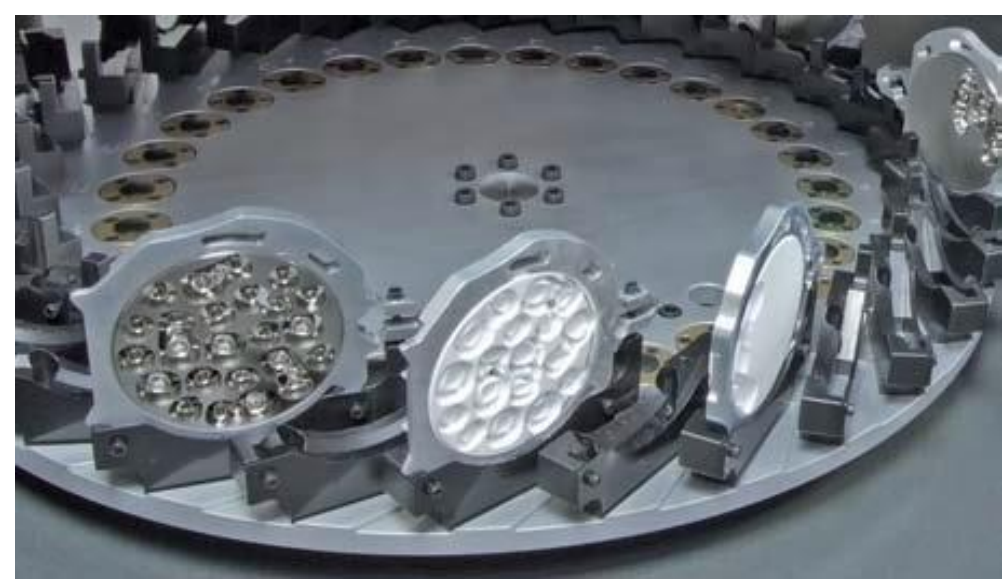

Abb. 3: Mit Rohlinghaltern bestückter Werkstückwechsler (http://www.wissner-gmbh.de/printmedien.htm), mit freundlicher Genehmigung der Wissner GmbH

Da aus einem Rohling bis zu 30 Restaurationen hergestellt werden können, ist es möglich, unbemannt 900 Versorgungen zu fräsen. Um die verschiedenen Materialien bearbeiten zu können, hat Wissner spezielle Rohlinghalter entwickelt (siehe Abb. 4), die es möglich machen, außer runden Discs auch Blöcke oder andere RohlingGeometrien zu verwenden.

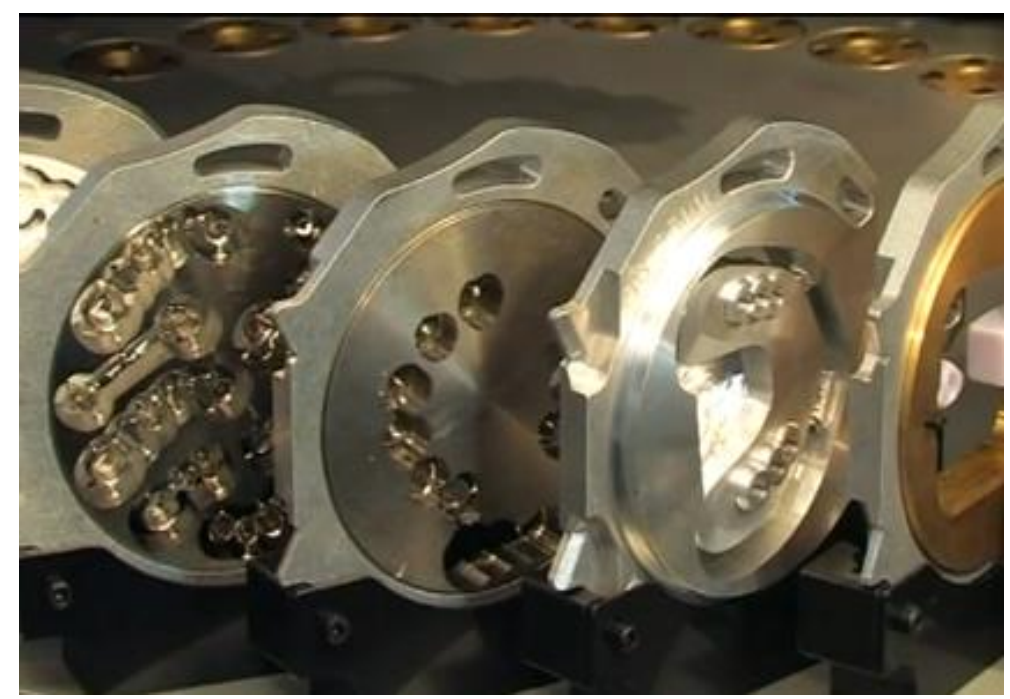

Abb. 4: Rohlinghalter mit unterschiedlichen Materialien. Von links: Titan, Kobalt-Chrom, Aluminium und Glaskeramik (Quelle: http://www.wissner-gmbh.de/Video/dental\%20video\%20imago.htm), mit freundlicher Genehmigung der Wissner GmbH

Zirkoniumdioxid und Gips werden trocken bearbeitet, wobei die anfallenden Späne durch ein Absaugverfahren abtransportiert werden. Es wird ein Luftwirbel (Zycloneffekt) erzeugt, in dessen Mitte ein Unterdruck entsteht, der die Späne der Absaugung zuführt. So setzt sich kein Staub in den empfindlichen Teilen der Geräte oder in den Werkzeugen fest und aufwändiges Reinigen der Maschine entfällt. 
Titan muss hingegen nass gefräst werden, da sonst der Fräser ausglühen würde. Hier kommt die Schwallkühlung zum Einsatz. Dieses Kühlsystem sorgt für eine gleichbleibende Temperatur des Fräsers und des Rohlings. Die Kühlflüssigkeit entfernt sofort die anfallenden Späne aus der Frässchneide des Werkzeugs und aus den Kavitäten des Werkstücks, um die Standzeit des Fräsers zu erhöhen.

Die Bearbeitung von Glaskeramik erfolgt mit diamantierten Schleifkörpern. Da auch bei dem Schleifen hohe Temperaturen entstehen, wird hier ebenfalls die Schwallkühlung eingesetzt.

Neben der Schwallkühlung gibt es die Minimalmengenkühlung, bei der ein dünner Schmierfilm um das Werkzeug gesprüht wird. Diese Minimalmengenkühlung kann bei Kobalt-Chrom-Arbeiten verwendet werden. Jedoch werden auch hier die Fräser mit der Schwallkühlung weniger beansprucht, sodass mit der Schwallkühlung längere Standzeiten erreicht werden.

Das Kühlmittelumlaufsystem mit eingebauter Filter- und Entsorgungseinheit führt dem System das Kühlmittel gereinigt wieder zu.

Mit den verschiedenen GAMMA-Systemen lassen sich viele verschiedene Indikationen abdecken. Es können zum Beispiel Inlays, Veneers, Kronen, Brücken und Implantate gefräst werden (CAM-Fans 2009).

\subsection{Dentale Werkstoffe für die CAD/CAM-basierte Verarbeitung}

\subsubsection{Titan}

Nachdem die erste Titanverbindung 1791 entdeckt wurde, dauerte es noch bis in die 1950er Jahre, bis der industrielle Einsatz von Titan möglich war (Kappert 1994).

Da Titan sehr reaktionsfreudig ist und vor allem mit Sauerstoff, Kohlenstoff, Stickstoff und Wasserstoff Verbindungen eingeht, sind die Möglichkeiten der Bearbeitung im zahntechnischen Labor bei Verfahren wie Gießen, Aufbrennen, Löten und Schweißen begrenzt.

Diese hohe Reaktionsfreudigkeit ist aber auch für die guten physikalischen und chemischen Eigenschaften des Titans verantwortlich. Es bildet sich in sehr kurzer Zeit eine stabile Oxidschicht, die unter anderem für die gute Biokompatibilität und die elektrochemische Beständigkeit verantwortlich gemacht wird (Kappert 1994). 
Mit der geringen Dichte von $4,50 \mathrm{~g} / \mathrm{cm}^{3}$ zählt Titan zu den Leichtmetallen. Sein spezifisches Gewicht ist halb so groß, wie das von Kobalt-Chrom-Legierungen und es ist $75 \%$ leichter als Goldlegierungen. Durch die gute Biokompatibilität und das niedrige spezifische Gewicht wird Titan im medizinischen Bereich oft für Hüft- oder Kniegelenkprothesen verwendet. Im zahnmedizinischen Bereich wird Titan vor allem für Implantate genutzt. Da Titan eine geringe Löslichkeit aufweist, ruft es keine Geschmacksveränderungen hervor und die niedrige Wärmeleitfähigkeit macht es außerdem zu einem geeigneten Restaurationsmaterial. Der Gießprozess ist allerdings sehr aufwändig. Durch die hohe Abkühlungsgeschwindigkeit und die hohe Viskosität der Titanschmelze muss bei dem Gussprozess eine Mindeststärke von $0,4 \mathrm{~mm}$ eingehalten werden. Um dünnere Wandschichten zu erreichen, muss in einem weiteren Arbeitsschritt nachgearbeitet werden (Cramer von Clausbruch 2005). Durch die Reaktionsfreudigkeit des Titans ist es im zahntechnischen Labor nicht möglich, eine von Reaktionsprodukten freie Oberfläche zu erstellen (Reppel et al. 1991).

Mit der CAD/CAM-Technologie ist Titan allerdings gut $\mathrm{zu}$ bearbeiten. Die Materialrohlinge werden industriell gefertigt, sodass die Werkstücke keine Verunreinigungen oder Lunker enthalten.

Bei den Randspalten kommen Studien bei dem Vergleich der Passungen von CAD/CAM-gefertigten Titan-Restaurationen mit den Passungen von konventionell hergestellten Titan-Kronen zu unterschiedlichen Ergebnissen. Leong et al. (1994) fanden bei beiden Herstellungsarten ungefähr gleich große Randspalten, bei Tan et al. (2008) und Han et al. (2011) zeigten die CAD/CAM-gefertigten Kronen eine schlechtere Passung. Trotzdem lagen die Randspalten der CAD/CAM-gefertigten Titan-Kronen unter der von McLean und von Fraunhofer (1971) postulierten Toleranzgrenze von $120 \mu \mathrm{m}$ (Besimo et al. 1997; Han et al. 2011; Karlsson 1993; Leong et al. 1994). Im Gegensatz zu anderen dentalen Legierungen ist Titan am Kronenrand für Röntgenstrahlung durchlässig, sodass kariöse Defekte auf Röntgenbildern besser erkannt werden können. Dies hilft bei der frühen Diagnose von Sekundär-Karies (Küpper und Marx 1993). Durch die Röntgentransparenz ist es außerdem möglich, eventuelle beim Guss entstandene poröse Stellen und Lunker festzustellen (Cramer von Clausbruch 2005). 
Die Lunkerkontrolle ist bei mit CAD/CAM-Technologie hergestellten Kronen nicht notwendig, da das Metallgefüge bei der Bearbeitung mit CAD/CAM-Systemen nicht verändert wird.

\subsubsection{Kobalt-Chrom-Legierungen}

Die Nichtedelmetalllegierungen (NEM-Legierungen) werden dank ihrer guten Biokompatibilität und den günstigeren Preisen häufig als Alternative zu Edelmetalllegierungen verwendet. Durch den höheren Elastizitätsmodul sind die NEM-Legierungen widerstandsfähiger gegen elastische Verformung, sodass auch weitspannige Brücken hergestellt werden können. Bei den hohen Temperaturen des Aufbrennens von Keramik können in Metallen Deformationen entstehen. Diese werden jedoch bei Kobalt-Chrom-Legierungen durch deren hohe Wärmefestigkeit verhindert (Geis-Gerstorfer 2005), sodass zur besseren Ästhetik keramische Verblendungen von NEM möglich sind.

Kobalt-Chrom-Legierungen bestehen zu etwa $67 \%$ aus Kobalt, zu $28 \%$ aus Chrom und ansonsten aus Elementen wie Molybdän, Wolfram, Mangan, Silizium und Eisen (Lenz 1998). Diese Legierung bildet zwei Phasen aus, den Mischkristall und die intermetallische Phase. Im hexagonal angeordneten Mischkristall fügen sich die größeren Chrom-Atome in das Kobaltgitter ein. Auf diese Weise werden die Gitterspannungen erhöht, was eine gesteigerte Festigkeit der Legierung hervorruft. Die intermetallische Phase hat ein tetragonales Raumgitter und erzeugt die für den Zahnersatz notwendige Härte und Sprödigkeit der Kobalt-Chrom-Legierung (Riquier und Krause 2011).

Kobalt als Hauptkomponente erzeugt die Dünnflüssigkeit der Schmelze und wirkt durch die Oxidbildung an der Oberfläche an der Passivierungsschicht mit.

Für die Mundbeständigkeit sind Chrom und Molybdän verantwortlich. Chromoxide tragen bei aufbrennfähigen Kobalt-Chrom-Legierungen außerdem zum metallkeramischen Verbund bei. Weitere Bestandteile dienen ebenfalls der vereinfachten Verarbeitbarkeit oder der Verbesserung physikalischer und chemischer Eigenschaften. 
Die nicht-aufbrennfähigen Kobalt-Chrom-Legierungen werden vor allem für herausnehmbare Modellgussprothesen verwendet. Durch den höheren Elastizitätsmodul, die größere Härte und das leichtere Gewicht sind die KobaltChrom-Legierungen bei diesem Zahnersatz den Edelmetallen überlegen.

Für die aufbrennfähigen Legierungen wurden die Modellgusslegierungen verändert, um den thermischen Ausdehnungskoeffizienten des Metalls an die Keramik anzupassen. Dieser Koeffizient sollte, da Keramik anfällig gegen Zugspannung ist, größer sein als der der Keramik. Auf diese Weise wird die Keramik beim Abkühlen unter eine leichte Druckspannung gesetzt (Geis-Gerstorfer 2005).

Trotz vieler Vorteile der NEM-Legierungen gegenüber den Edelmetalllegierungen, wie ein höherer Elastizitätsmodul, geringere Dichte, niedrigere Wärmeleitfähigkeit und der niedrigere Preis, wurden Kobalt-Chrom-Legierungen anfangs seltener eingesetzt. Die Passgenauigkeit der Restaurationen war ungenügend (Hohmann 1984; Kobes et al. 1988). Mittlerweile erreichen die gegossenen KobaltChrom-Kronen jedoch einen ebenso guten Randschluss wie Edelmetallrestaurationen (Schaudig et al. 2009).

\subsubsection{Zirkoniumdioxid}

Durch seine hohe Bruch- und Verschleißfestigkeit, Korrosionsbeständigkeit und niedrige Wärmeleitfähigkeit wird Zirkoniumdioxid häufig für metallfreien Zahnersatz in stärker kaubelasteten Bereichen eingesetzt.

Zirkoniumdioxid liegt bei Raumtemperatur in der monoklinen Kristallform vor, die sich bei ungefähr $1100^{\circ} \mathrm{C}$ reversibel in eine tetragonale Kristallform umwandelt. Ein weiterer Wandel der Kristalle in die kubische Form vollzieht sich bei etwa $2300^{\circ} \mathrm{C}$.

Durch die Zugabe von 3-5\% Yttriumoxid kehrt das Zirkoniumdioxid beim Abkühlen nicht in die monokline Form zurück, sondern verbleibt in der tetragonalen Phase. Da bei der Umwandlung von der tetragonalen in die monokline Phase ein Volumenzuwachs von ca. 3-5\% eintritt, steht das Y-TZP (Yttrium Stabilized Tetragonal Zirconiumdioxide Polycrystals = Yttriumoxid teilstabilisiertes, tetragonales polykristallines Zirkoniumdioxid) unter Vorspannung (Janda 2007b). 
Bei dem Auftreten eines Risses führen die Tangentialspannungen an den Rissspitzen zur Umbildung der tetragonalen Phase in die monokline Phase. Durch die Volumenvergrößerung entstehen an der Rissspitze Druckspannungen, sodass das Risswachstum gehemmt bzw. gestoppt wird (Cramer von Clausbruch 2004; Janda 2007a).

Durch eine spezielle Herstellungsweise, dem heiß-isostatischen Pressen (HIP = Hot Isostatically Pressed) tritt eine weitere Festigkeitssteigerung ein.

Die Keramikrohlinge können als Grünling bzw. als Weißling oder im oben genannten geHIPten Zustand erworben werden.

Die Grünlinge sind nicht wärmebehandelt. Dem Keramikpulver wird ein Bindemittel zugeführt, sodass es in die erwünschte Gestalt gebracht werden kann. Die Grünlinge sind gut durch Fräser zu bearbeiten, dürfen jedoch trotz Staubentwicklung nur trocken gefräst werden. Das Kühlmittel würde das Bindemittel entfernen, sodass das Material erweichen würde. Da bei der anschließenden Sinterung eine Sinterschwindung von 30\% eintritt, muss das Werkstück um diese Prozentzahl vergrößert ausgefräst werden. Der Vorteil der Grünlinge ist die leichte Bearbeitbarkeit. Daraus resultieren längere Standzeiten der Werkzeuge. Allerdings führt die geringe Festigkeit auch zu Schwierigkeiten beim Transport.

Weißlinge sind vorgesinterte Keramikrohlinge. In einem Vorbrand wird die Keramik vorverfestigt, um das Verarbeitungsrisiko zu mindern und einen sicheren Transport zu ermöglichen. Bei diesem Vorbrand erfährt die Keramik bereits eine Sinterschwindung von ca. $5 \%$.

Die als Weißbearbeitung bezeichnete Verarbeitung kann trocken oder mit Kühlmittel erfolgen. Der Schleifstaub kann durch das Kühlmittel abgeleitet werden und die mechanische Belastung der Werkzeuge durch den Reibungsverschleiß sinkt. Um die Endfestigkeit der Werkstücke zu erreichen, werden sie sechs bis zehn Stunden bei bis $\mathrm{zu} 1500^{\circ} \mathrm{C}$ gesintert. Durch die Volumenminderung beim Vorbrand beträgt die Schwindung bei dem endgültigen Sintern der Weißlinge ca. 25\% (siehe Abb. 5). 


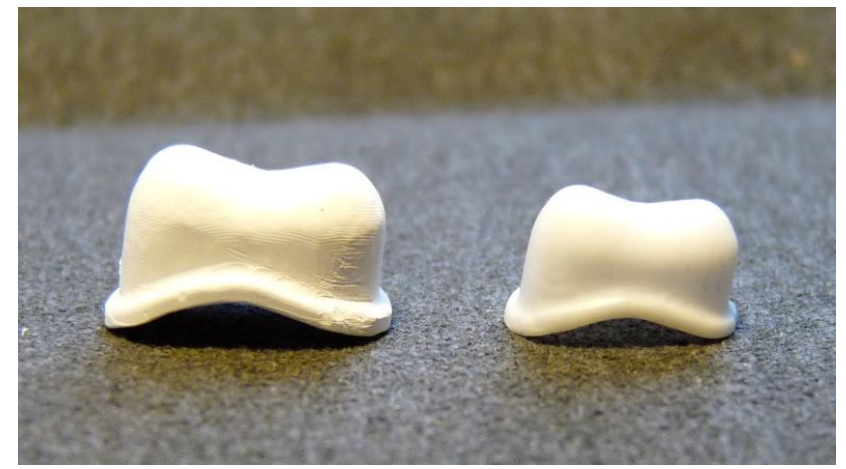

Abb. 5: Zirkoniumdioxid-Käppchen vor dem Sintern (links) und nach dem Sintern (rechts)

Um geHIPte Rohlinge herzustellen, wird die Keramik nach dem industriellen Standard dichtgesintert und in einem nachfolgenden Prozess weiter verdichtet. Dadurch entstehen eine maximale Materialdichte und weniger Fehlstellen. Auf diese Weise werden die mechanischen und optischen Eigenschaften verbessert.

Die erhöhte Biegefestigkeit und Bruchzähigkeit ermöglichen es, Gerüste mit bis zu 14 Brückengliedern herzustellen (Janda 2007a). Die ausgefrästen Restaurationen werden nach der Bearbeitung nicht erneut gesintert, sodass die Fertigung im Maßstab 1:1 erfolgt. Eventuelle Ungenauigkeiten bei der Sinterschrumpfung haben demnach keine Auswirkungen auf die Passgenauigkeit der Restauration.

Der Vorteil der Keramiken gegenüber den dentalen Metallegierungen ist ihre Transluzenz. Obwohl das Licht die Keramik besser durchdringen kann als das Metall, müssen konventionelle Zirkoniumdioxid-Gerüste aufgrund ihrer hohen Opazität ebenfalls verblendet werden. Doch im Gegensatz zu den Metallen scheint das Zirkoniumdioxid nicht gräulich durch die Verblendkeramik hindurch. Daher sind hiermit Restaurationen hoher ästhetischer Qualität möglich.

Zirkoniumdioxid-Gerüste haben sich auch im Seitenzahnbereich bewährt (Belli et al. 2011; Rinke und Fischer 2012) und führen dort zu vergleichbaren klinischen Ergebnissen wie die verblendeten Metallgerüste (Vigolo und Mutinelli 2012). Obwohl die Verblendkeramik mit der Gerüstkeramik eine stabile Verbindung eingeht (Tinschert et al. 1999), kommt es vor, dass die Verblendkeramik abplatzt oder abschilfert (chipping). Dieses Problem ist zum Teil behandlerabhängig (Kerschbaum et al. 2009) und kann durch die richtige Verarbeitung der Keramik und materialgerechte Präparation des Zahnes gering gehalten werden (Pospiech 2010). 
Um die Problematik des Chippings zu umgehen, wurden transluzentere Zirkoniumdioxidvarianten entwickelt. Diese Keramiken können für monolithische Kronen und Brücken im Seitenzahnbereich eingesetzt werden (Rinke und Fischer 2012). Da eine Verblendung der vollanatomisch ausgefrästen Restaurationen nicht nötig ist, muss bei der Präparation der Zähne weniger Zahnhartsubstanz entfernt werden. Unabhängig vom Design der Präparationsgrenze ist ein zirkulärer Substanzabtrag von 0,4 $\mathrm{mm}$ ausreichend (Aggstaller et al. 2006).

Nach McLean und von Fraunhofer (1971) sind klinisch erfolgreiche Restaurationen möglich, wenn der Randspalt unter 120 um liegt. Setzt man also einen Randspalt von maximal $120 \mu \mathrm{m}$ als vertretbar voraus, liegen die Passungen der Vollkeramikrestaurationen im klinisch akzeptablen Bereich (Bindl und Mörmann 2005; Kokubo et al. 2005; Lee et al. 2008; Reich et al. 2008a; Reich et al. 2008b). Die Passung verändert sich auch nach Aufbrennen der Keramik oder nach dem Glanzbrand nicht (Vigolo und Fonzi 2008).

\subsubsection{Glaskeramik}

Aufgrund ihrer geeigneten Transluzenz und der guten Einfärbbarkeit werden Glaskeramiken häufig für hochästhetischen Zahnersatz verwendet.

Der Ursprungsstoff der Glaskeramiken besteht aus Feldspat, Quarz und Kaolin und hat einen amorphen Aufbau. Diese amorphe Struktur wird durch die Zugabe kristallbildender Substanzen verfestigt (Marxkors et al. 2008). Die Kristalle wachsen jedoch nicht isoliert oder zufällig in der Glasmatrix, da sonst ein uneinheitlicher Aufbau mit unzureichenden Eigenschaften entstehen würde. Durch Temperaturänderungen können die Kristallgrößen und die Anzahl der Kristalle beeinflusst werden. Das Ausmaß, die Form, die Vernetzung der Kristallphasen und die resultierende Restglasphase bestimmen die Merkmale der Glaskeramik. Mit den Temperaturbehandlungen können zum Beispiel die mechanische Festigkeit gesteigert, die Wärmedehnung bestimmt und die optischen und elektrischen Eigenschaften beeinflusst werden (Bürke 2006). 
Um den Glaskeramikrohling im CAD/CAM-Verfahren wirtschaftlich bearbeiten zu können, wird er in einer Zwischenstufe gefräst. Der Rohling besitzt in dieser Phase eine gute Homogenität und eine ausreichende Festigkeit. Die in dieser Arbeit verwendeten Rohlinge (IPS e.max CAD von Ivoclar vivadent, Schaan Liechtenstein) haben vor dem Sintern eine bläulich-graue Färbung. Nach der Bearbeitung des Werkstücks findet im Keramikofen ein Kristallisationsprozess statt. Bei diesem 30minütigen Vorgang entstehen die endgültige Festigkeit und die optischen Eigenschaften (Hummel und Seger 2005). Die bläuliche Färbung lässt nach und die gewünschte Zahnfarbe entwickelt sich (siehe Abb. 6).

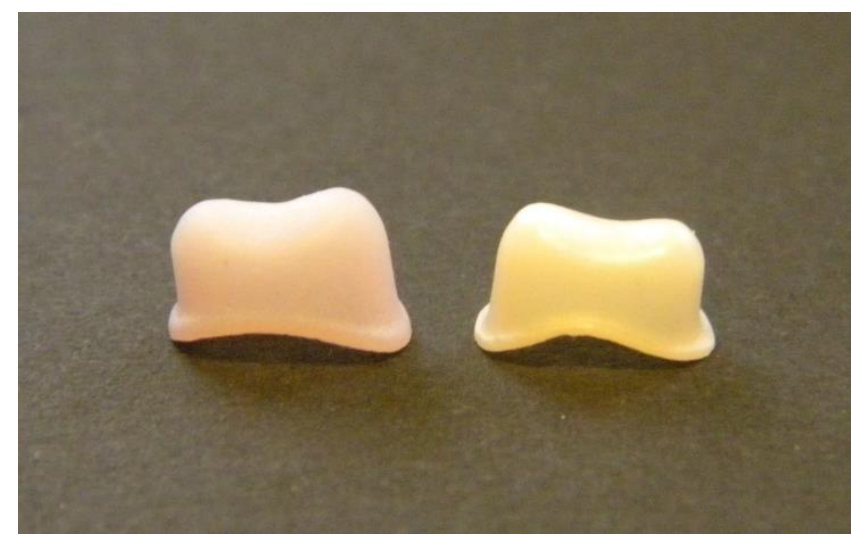

Abb. 6: Glaskeramik vor dem Sintern (links) und nach dem Sintern (rechts)

Im Gegensatz zur Sinterung von Zirkoniumdioxid-Werkstücken bleibt bei der Glaskeramik in diesem Schritt der Sinterschwund aus, die Restaurationen können deshalb in Originalgröße ausgefräst werden.

Glaskeramik eignet sich besonders für Einzelzahnkronen im Seitenzahnbereich und für Brücken mit bis zu drei Brückengliedern im Frontzahnbereich (Bürke 2006; Hummel und Seger 2005). Durch die Sprödigkeit der Glaskeramik sind ausgedehntere Restaurationen bisher nicht möglich. 


\subsubsection{Sonstige Werkstoffe}

Weitere Materialien, die mit der dentalen CAD/CAM-Technik bearbeitet werden können, sind zum Beispiel Kunststoffe und Wachs. Da diese Materialien keine Verwendung für definitive Restaurationen finden, wird innerhalb dieser Arbeit auf Kunststoffe und Wachs nur kurz eingegangen und ihre Randpassungen werden nicht untersucht.

Die Kunststoffe werden vor allem für die Herstellung von provisorischen Versorgungen verwendet. Ein Provisorium wird für den Schutz des Zahns für die Zeit zwischen der Präparation und der Eingliederung der definitiven Versorgung angefertigt. Es bewahrt das Dentin und die Pulpa des präparierten Zahns vor thermischen, chemischen und physikalischen Einflüssen. Außerdem soll es den Zahn, die Nachbarzähne und die Okklusion stabilisieren (Czerny 2003).

Die konventionellen Kunststoffprovisorien werden vom Zahnarzt (Direktprovisorium) oder Zahntechniker (Eierschalenprovisorium) angefertigt. Der Zahnarzt befüllt einen vorher genommenen Abdruck mit zähflüssigem Kunststoff, der im Mund des Patienten aushärtet. Das Eierschalenprovisorium wird vom Zahntechniker im Dentallabor hergestellt und vom Behandler am Patienten unterfüttert.

Für die dentale CAD/CAM-Technologie werden Rohlinge aus auspolymerisiertem Kunststoff angeboten. Da diese Kunststoffe industriell gefertigt werden, können die Eigenschaften besser gesteuert und geprüft werden. Das Ausmaß an nicht umgewandelten Monomeren (Restmonomergehalt) bleibt gering und das Allergierisiko sinkt (Stawarczyk et al. 2010).

CAD/CAM-gefertigte Kunststoffprovisorien sind besonders für Langzeitprovisorien geeignet. Diese kommen zum Einsatz, wenn vor der Eingliederung der definitiven Versorgung die Kieferrelation oder die Okklusion neu gestaltet werden muss (Edelhoff et al. 2012). Die veränderte Situation kann risikoarm über mehrere Wochen erprobt und bei Bedarf korrigiert werden. Auch eine Umgestaltung der Ästhetik und Phonetik kann vom Patienten getestet werden.

Da die Langzeitprovisorien oft über Monate getragen werden, müssen sie eine gute Mundbeständigkeit und geeignete mechanische Eigenschaften besitzen (Fischer 2008). 
Die Kaukraft im Molarenbereich kann $380 \mathrm{~N}$ erreichen (Helkimo et al. 1977), sodass die Kunststoffe hohe Bruchlastwerte aufweisen müssen. Bei Studien erreichten die CAD/CAM-gefertigten Provisorien signifikant bessere Ergebnisse als die Direktprovisorien (Stawarczyk et al. 2008; Stawarczyk et al. 2010).

Wachse werden vor allem für die Gusstechnik verwendet. Auf dem Gipsmodell wird die zu erstellende Restauration mit Modellierwachs aufgewachst. Dafür wird der Wachs verflüssigt und tropfenweise auf den Gips aufgetragen. Durch stückweises Anfügen und Abtragen des Wachses wird der Zahnersatz geformt. Ist die Modellation beendet, wird sie in einer feuerfesten Masse eingebettet. Nach dem Abbinden der Einbettmasse, wird sie in einem Ofen vorgeheizt und das Wachs wird rückstandsfrei ausgebrannt. Auf diese Weise entsteht eine Hohlform (lost-wax-Prinzip), in die im nächsten Schritt das Metall gegossen werden kann. Im Anschluss an das Ausbetten muss die Restauration ausgearbeitet und poliert werden.

Mit einigen dentalen CAD/CAM-Systemen können auch Wachse gefräst werden. Dementsprechend erübrigt sich die aufwändige Wachsmodellation. Ebenso entfällt die Gefahr des Verzugs, der bei Temperaturschwankungen während des Aufwachsens entstehen kann.

\subsection{Relevanz der marginalen Passgenauigkeit}

Wie bereits am Anfang dieser Arbeit erwähnt, bestimmen nicht nur die Ästhetik und die mechanischen Eigenschaften der Materialien die Qualität einer festsitzenden Versorgung, sondern auch die Güte des marginalen Randschlusses.

Holmes et al. (1989) haben eine Terminologie zur Passgenauigkeit eingeführt (siehe Abb. 7), da zuvor gleiche Bezeichnungen für verschiedene Messstrecken verwendet wurden und die Vergleichbarkeit der Studien eingeschränkt war. 
In der vorliegenden Arbeit wird der so genannte Randschluss (Strecke B) gemessen.
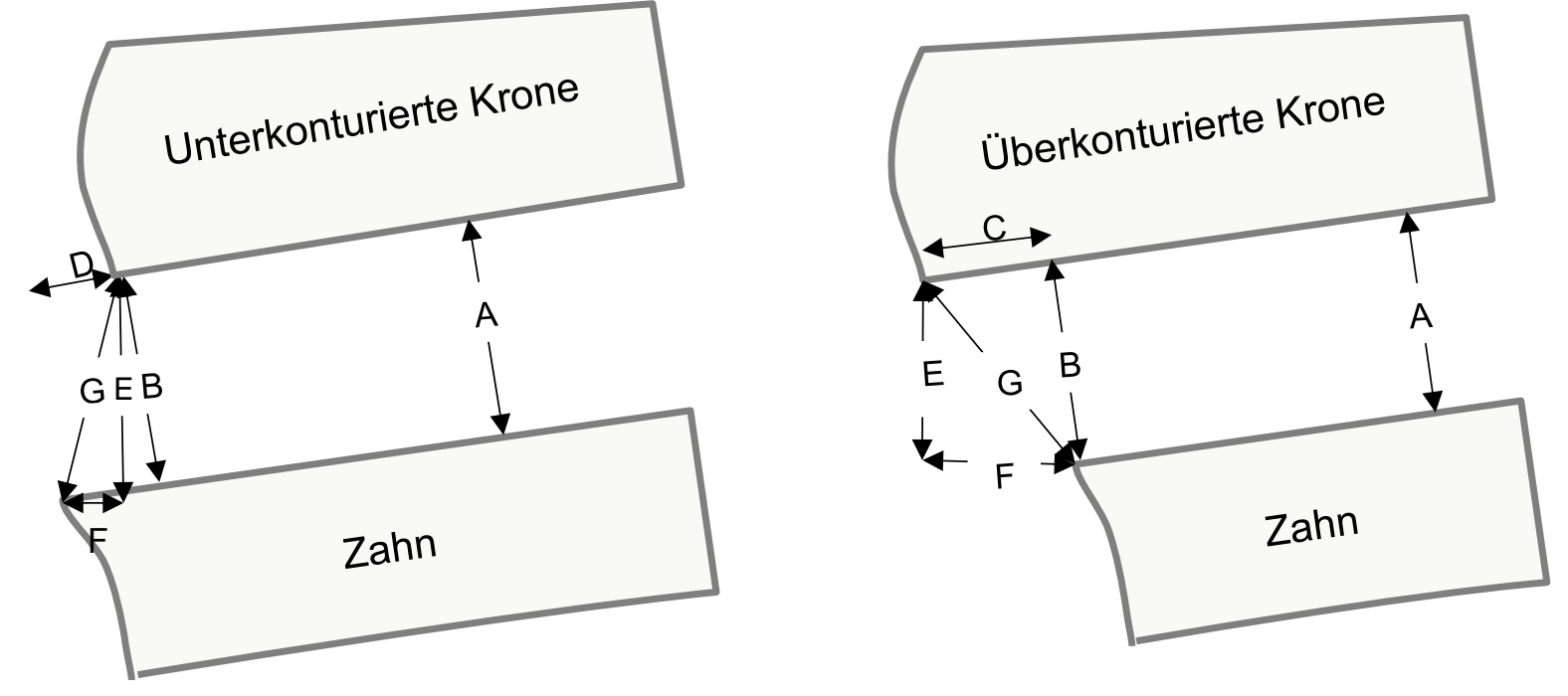

\begin{tabular}{|l|l|}
\hline A & Innerer Spalt \\
B & Randschluss (im $90^{\circ}$-Winkel zu C bzw. D) \\
C & Überextendierter Rand \\
D & Unterextendierter Rand \\
E & Vertikale marginale Diskrepanz (im $90^{\circ}$-Winkel zu F) \\
F & Horizontale marginale Diskrepanz \\
G & Absolute marginale Diskrepanz \\
\hline
\end{tabular}

Abb. 7: Darstellung und Terminologie der Messstrecken (Darstellung modifiziert nach Holmes et al. 1989: J Prosthet Dent $\underline{62}$ S. 406)

In der Theorie liegt ein exakter Randschluss vor (B, C, D, E, F und G = 0), wenn der Zahnersatz dem präparierten Zahn an allen Seiten glatt anliegt (Rehberg 1971).

Diese Perfektion kann jedoch im klinischen Alltag schon durch das Befestigen der Krone mit Zement nicht erreicht werden. Es entsteht eine Zementschicht zwischen Zahnersatz und Zahnstumpf (Demmel 1971; Stappert et al. 2001). Daher kann auch ein Randspalt von $20 \mu \mathrm{m}$ als exakter Randschluss bezeichnet werden (Rehberg 1971). Als klinische Toleranzgrenze werden $120 \mu \mathrm{m}$ angesehen, da erfolgreiche Restaurationen auch bei dieser Randspaltweite noch möglich sind (McLean und von Fraunhofer 1971). 
Zwar wird auch ein größerer Randspalt zunächst durch den Befestigungszement verschlossen, doch mit Zunahme der Spaltbreite vergrößert sich durch die Zementauswaschung auch die Spalttiefe (Schwickerath 1979). In diesem Spalt lagert sich Plaque ab (Geurtsen 1990; Kraft 1969), sodass unter $75 \%$ der abstehenden Kronenränder auch Konkrementablagerungen zu finden sind (Spiekermann 1986).

Das führt dazu, dass ein ungenügender Randschluss häufig mit pathologischen Reaktionen des Parodontiums wie Gingivitiden, erhöhten Taschentiefen und Parodontiden einher geht (Felton et al. 1991; von Gropp und Schwindling 1971; von Hüttner 1971; Kraft 1969; Rehberg 1971).

Durch Röntgenkontrollen können fehlerhafte Kronen erkannt werden, da ein röntgenologisch insuffizient aussehender Kronenrand auch klinisch ungenügend ist. Jedoch werden umgekehrt klinisch mangelhafte Kronenränder röntgenologisch häufig nicht erkannt (Spiekermann 1986).

Die übliche klinische Untersuchung des Kronenrands erfolgt durch Sondierung. Wird die Sonde senkrecht über den Randspalt geführt, können Randungenauigkeiten von $20 \mu \mathrm{m}$ wahrgenommen werden. Dies ist jedoch im Interdentalraum und unter dem Zahnfleischsaum nicht realisierbar (Rehberg 1971).

Die Studien von Kerschbaum und Porschen (1998) und von Spiekermann (1986) zeigen, dass Randungenauigkeiten, wie Über- und Unterkonturierungen oder zu lange oder kurze Kronenränder sehr häufig vorkamen. Andere Studien ermitteln jedoch in in-vivo und in-vitro-Versuchen, dass klinisch akzeptable Werte für die Randspalten der Restaurationen, die in Gussverfahren oder mit CAD/CAM-Techniken hergestellt wurden, erreicht werden können (Bindl und Mörmann 2005; Karlsson 1993; Kokubo et al. 2005; Schaudig et al. 2009; Tan et al. 2008; Vanlioglu et al. 2012). 


\subsection{Präparationsgrenze}

\subsubsection{Klinische Bedeutung der Präparationsgrenze}

Die Voraussetzung für eine gute Randpassung ist eine geeignete Präparationstechnik. Hierbei wird nicht nur auf einen angemessenen Substanzabtrag besonderen Wert gelegt, sondern auch die Form und Lage der Präparationsgrenze spielen eine wichtige Rolle.

Um die Ausbildung von Sekundär-Karies hinauszuzögern, wurde der Kronenrand häufig tief in der Zahnfleischtasche platziert. Die Verletzungen des Gewebes führten jedoch zu Gingivarezessionen und irreversiblem Substanzverlust des Knochens (Kraft 1969). Da an subgingivalen Restaurationsgrenzen auch bei regelmäßigem Recall stärkere gingivale Entzündungen und tiefere Taschen zu finden waren (Bader et al. 1991), werden heutzutage supragingivale Kronenränder bevorzugt. Allerdings ist eine supragingivale Präparationsgrenze in weniger als 30\% der Fälle möglich (Gernet und Mitteldorf 1984). Die Präparationsgrenze muss durch tiefe Karies oder Füllungsränder, zu kurze klinische Kronen, eingeschränkte Ästhetik oder bereits vorhandene subgingivale Präparationsgrenzen häufig in den Sulkus gelegt werden.

Erfolgt die intrasulkuläre Präparation atraumatisch und höchstens $1,5 \mathrm{~mm}$ tief im Sulkus, werden bei gutem Randschluss, gründlicher Zementrestentfernung und guter Mundhygiene keine pathologischen Reaktionen des Parodontiums festgestellt (Ariely 1979; Günay et al. 2001).

Bei der Form der Präparationsgrenze lassen sich vier unterschiedliche Typen unterscheiden:

Tangentialpräparation, Hohlkehlpräparation, Stufenpräparation und die Stufenpräparation mit Abschrägung. 


\subsubsection{Präparationsformen und -geometrien}

\subsubsection{Tangentialpräparation}

Bei dieser Präparationsart wird ein spitz zulaufender Diamant genutzt. Durch den fast stufenlosen Übergang von präpariertem Zahn zu nicht präparierter Zahnhartsubstanz erfolgt in diesem Bereich ein geringer Substanzabtrag (siehe Abb. 8). Durch das geringe Platzangebot ist es jedoch oft nicht möglich, den Restaurationsrand ebenmäßig der Anatomie des Zahns anzugleichen. Da für den Zahntechniker die Präparationsgrenze häufig nicht zu erkennen ist (Spiekermann 1986), entstehen Ungenauigkeiten am Kronenrand.

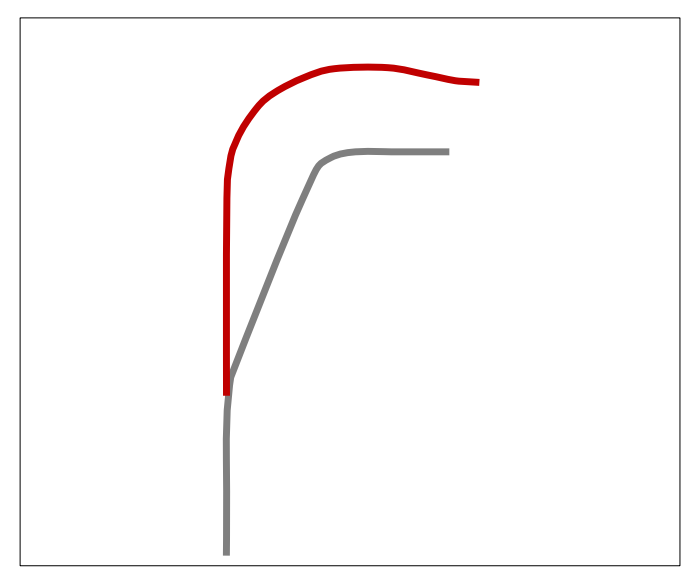

Abb. 8: Schematische Darstellung einer Tangentialpräparation

Der Kronenrand bei einer Tangentialpräparation ist dünn auslaufend. Besteht ein solcher Kronenrand aus Keramik, frakturiert diese häufig schon beim Einsetzen der Restauration (Marxkors 2009).

\subsubsection{Hohlkehlpräparation}

Für eine Hohlkehlpräparation wird ein so genannter "torpedoförmiger" Diamant verwendet. Durch die Form des Diamanten wird zirkulär eine Hohlkehle gebildet (siehe Abb. 9). Auf diese Weise wird die Präparationsgrenze deutlich gekennzeichnet (Lehmann et al. 2009). 


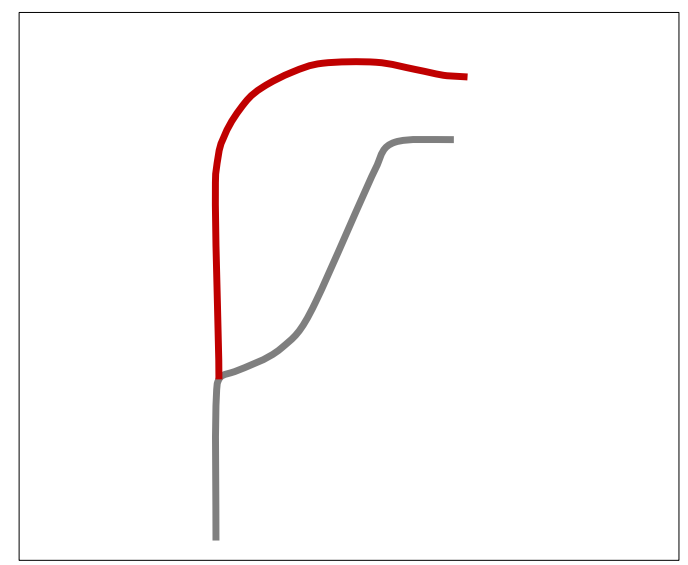

Abb. 9: Schematische Darstellung einer Hohlkehlpräparation

Aufgrund der einfachen technischen Durchführung wird die Hohlkehlpräparation sehr häufig verwendet. Da zervikal ein ausreichender Substanzabtrag gewährleistet ist, kann sie sowohl bei Metall- als auch bei Keramikkronen genutzt werden.

\subsubsection{Stufenpräparation}

Hier wird zervikal eine nach innen gerundete Schulter mit ungefähr 0,5-1 mm Breite angelegt (Lehmann et al. 2009). Die Präparationsgrenze ist somit gut erkennbar (siehe Abb. 10). Zervikal ist genug Platz für eine Verblendung, sodass kein störender Metallrand sichtbar wird (Marxkors 2009).

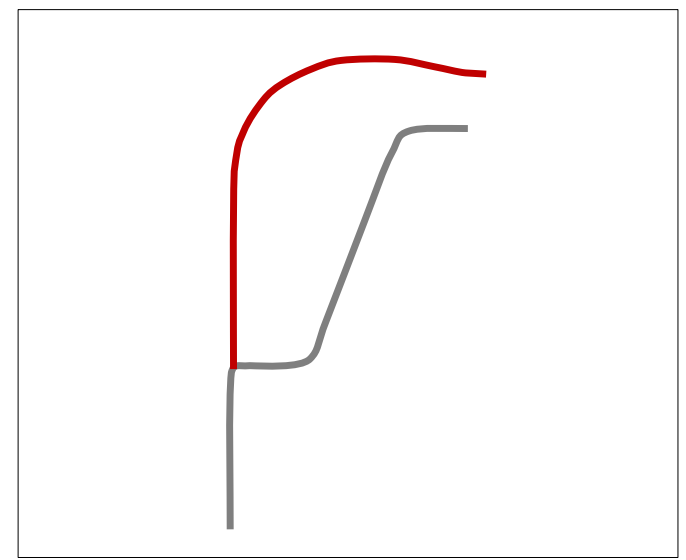

Abb. 10: Schematische Darstellung einer Stufenpräparation 
Die horizontale Auflage für die Drucklastaufnahme und die abgerundeten Formen verringern Spannungsüberhöhungen in der Keramik (Pospiech 2010), sodass höhere Bruchwerte für Keramiken entstehen (Aggstaller et al. 2006).

Durch die breite zervikale Stufe ist jedoch der Substanzabtrag erhöht, sodass im Vergleich zu anderen Präparationsarten bei der Stufenpräparation eine geringere Stärke an Restdentin verbleibt (Borelli et al. 2013). Die geringere Restdentinstärke resultiert in einer häufigeren Schädigung der Pulpa (Wisithphrom et al. 2006).

\subsubsection{Stufenpräparation mit Abschrägung}

Bei dieser Präparationsart wird die bei der Stufenpräparation angelegte Schulter zusätzlich abgeschrägt (siehe Abb. 11).

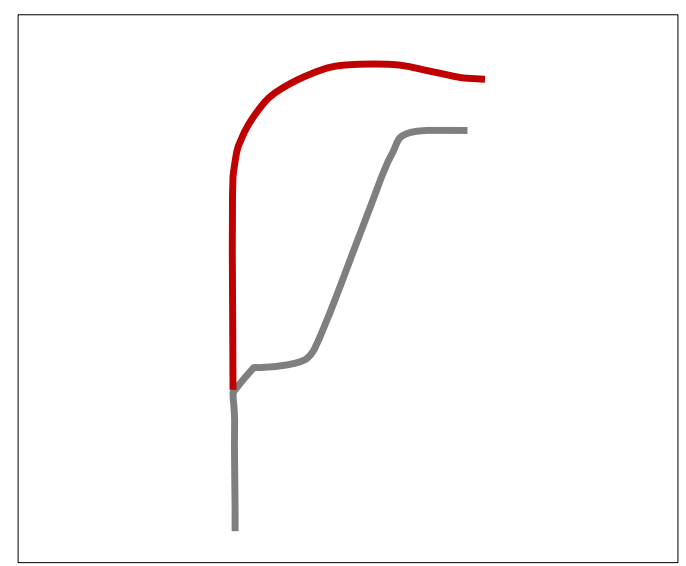

Abb. 11: Schematische Darstellung einer Stufenpräparation mit Abschrägung

Durch den kleineren Winkel soll ein kleinerer Randspalt im Vergleich zur Stufenpräparation erreicht werden. Allerdings ist bei zu steilen Abschrägungen der Übergang zwischen Präparation und Zahn wie bei der Tangentialpräparation schlecht sichtbar (Lehmann et al. 2009). Die technische Durchführung ist im klinischen Alltag im Gegensatz zu den anderen Präparationsarten erschwert, sodass diese Präparationsart nur noch bei Vollguss-Teilkronen angewendet wird. 


\section{Material und Methoden}

\subsection{Herstellung des Masterstumpfs}

Der für diese Arbeit verwendete Originalstumpf (Masterstumpf) wurde für eine vorhergehende Arbeit (Fornefett 2010) erstellt. Er wurde entsprechend zu einem Oberkiefer-Prämolaren präpariert und aus einer Metalllegierung (Palliag $M$, DeguDent, Hanau) hergestellt. Die Schnitttiefe von $1 \mathrm{~mm}$ zirkulär und $1,5 \mathrm{~mm}$ okklusal richtete sich nach den Vorgaben für den Abtrag bei Vollkeramik- und VMKKronen-Präparationen. Als Präparationsgrenze wurde eine innen abgerundete zirkuläre Stufe gewählt, die nach klinischen und anatomischen Anforderungen approximal weiter koronal verlief als vestibulär und palatinal. Die vestibluläre und die palatinale Höckerwand waren jeweils um $2^{\circ}$ geneigt, sodass der Konuswinkel $4^{\circ}$ betrug. Es wurde darauf geachtet, dass die anatomische Grundform des Zahnes 25 grundlegend erhalten blieb und keine unter sich gehenden Bereiche auftraten. Die präparierten Flächen wurden geglättet und abgerundet, damit möglichst wenig negative Auswirkungen auf den späteren Reproduktions-, Scan- oder Herstellungsprozess entstanden.

Für diesen Versuch wurde ein Oberkiefer Prämolar 25 der Firma frasaco (Tettnang) präpariert. Die vorgesehene Präparationstiefe von $1 \mathrm{~mm}$ wurde parallel zur Zahnachse im Bereich der Zahnkrone mit einem parallelen und vor Kopf abgerundeten, $1 \mathrm{~mm}$ starken, diamantierten Schleifkörper markiert. Okklusal wurden 1,5 mm tiefe Markierungsrillen eingeschliffen. Im Anschluss daran wurde der Zahn in einem Fräsgerät mit einem konischen, vor Kopf abgerundeten Hartmetallfräser zirkulär entsprechend der Markierungsrille und dem Zahnfleischverlauf beschliffen. Als Präparationsgrenze wurde eine akzentuierte Hohlkehle mit $1 \mathrm{~mm}$ Schnitttiefe gewählt. Anschließend erfolgte die okklusale Reduzierung um 1,5 mm.

Der bearbeitete Stumpf wurde an der Präparationsgrenze gekürzt und mit Fräswachs ergänzt, um den Stumpfsockel in apikaler Richtung mit einem in ein Parallelometer eingespannten Wachsschaber zu parallelisieren.

Anschließend wurde der präparierte Prämolar mit der Präzisionsabformmasse Adisil rapid (SILADENT Dr. Böhme \& Schöps GmbH, Goslar) abgeformt. 
In die geschaffene Negativform wurde eine dünne Schicht rückstandslos verbrennender Modellierkunststoff (GC Pattern Resin LS, GC Europe N.V., Leuven, Belgien) eingebracht und der verbliebene Hohlraum mit Gusswachs gefüllt. Am unteren Ende des wächsernen Stumpfsockels wurde eine Fußplatte modelliert und daraufhin nach der Modellgusstechnik angestielt. Im Anschluss daran wurde das Gussobjekt in eine phosphatgebundene Präzisionseinbettmasse (rema dynamic S, Dentaurum, Ispringen) eingebettet.

Das Gusswachs und der Modellierkunststoff wurden ausgebrannt, sodass die entstandene Hohlform bei ungefähr $1040^{\circ} \mathrm{C}$ mit der Nicht-Edelmetalllegierung Palliag M (DeguDent, Hanau) ausgegossen werden konnte. Nachdem der Metallstumpf ausgebettet worden war, wurde er abgestrahlt und vom Gusskanal getrennt. Daraufhin wurde die axiale Neigung auf $2^{\circ}$ mit einem konischen und vor Kopf abgerundeten Hartmetallfräser im Fräsgerät nachbearbeitet. Als der Stumpf eine innen abgerundete Stufe mit einer zirkulären Präparationstiefe von $1 \mathrm{~mm}$ aufwies, wurde er mit einem Degusit-Stein am Fräsgerät geglättet und poliert, bis alle scharfen Kanten und Ecken entfernt waren. Zum Schluss wurde der Stumpf hochglanzpoliert (Fornefett 2010).

\subsection{Herstellung der Meistermodelle (Replika)}

Zur Abformung des Masterstumpfs wurde ein Polyetherabformmaterial (Impregum Penta, 3M ESPE AG, Seefeld) genutzt. Polyether gehören wie die Alginate und Silikone zu den irreversibel-elastischen Abformwerkstoffen. Sie sind wie die additionsvernetzenden Silikone sehr dimensionsstabil und besitzen eine hohe Zeichnungsschärfe, heben sich jedoch auch durch ihre große Endhärte hervor. Impregum wird als Paste-Paste-System angeboten, welches fast ausschließlich maschinell (PENTAMIX) angemischt wird. Da diese Abdrücke sehr genau sind, werden sie im klinischen Alltag vor allem für die Abformung von festsitzendem Zahnersatz auf dentalen Pfeilern oder Implantaten genutzt.

Die Polyethermassen eignen sich der Einphasentechnik entsprechend am besten zur gleichzeitigen Verwendung von Spritze und individuellem Löffel. In dieser Studie wurde das Material mit der Spritze um den metallischen Stumpf herum aufgetragen. 
Gleichzeitig wurde ein individueller Löffel mit dem gleichen Material gefüllt und anschließend über den Stumpf gebracht, um eine gleichmäßige Schichtdicke des Abformmaterials zu garantieren. Nach der Abbindezeit von fünf Minuten wurde der Masterstumpf aus dem Abdruck herausgenommen.

Im Anschluss an die vom Hersteller des Abformmaterials vorgegebene Rückstellzeit von mindestens 30 Minuten konnten die Abdrücke mit Gips ausgegossen werden.

Zur Herstellung der Meistermodelle (Replika) wurde der Superhartgips GC FujiRock EP (Superhartgips der Klasse 4, GC Europe N.V., Leuven, Belgien) verwendet. Dieser Gips wurde bei einem Anmischverhältnis von $100 \mathrm{~g}$ Pulver zu $20 \mathrm{ml}$ destilliertem Wasser unter Vakuum angerührt.

Der Hersteller gibt eine Abbindexpansion von ca. 0,08\% und eine Druckfestigkeit von $35 \mathrm{MPa}$ an.

Das Ausgießen der 20 Abformungen fand auf einer Rüttelplatte statt, um ein blasenfreies Ausfließen des thixotropen Gipses zu ermöglichen. Nachdem der Gips ausgehärtet war, wurden die Replika aus den Abdrücken genommen, die Sockel getrimmt und die Modelle durchnummeriert (siehe Abb. 12).

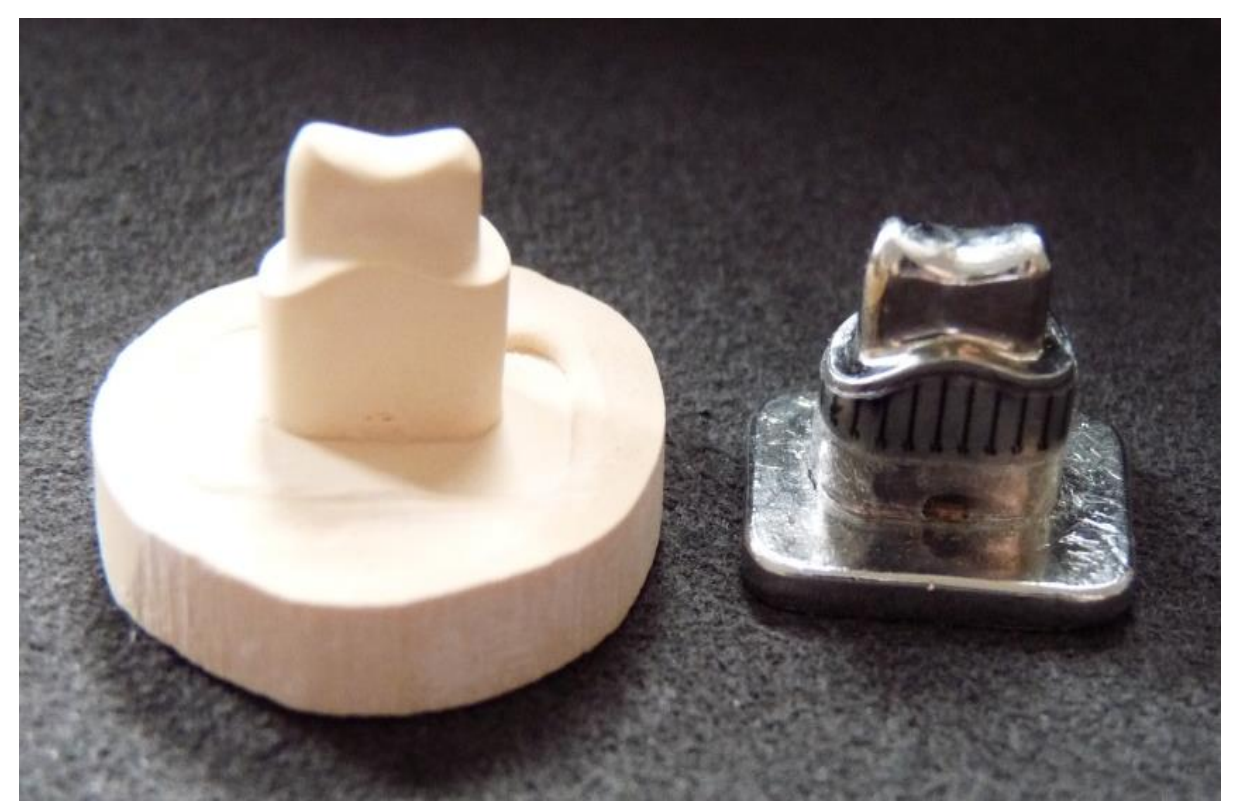

Abb. 12: Meistermodell (links) und Masterstumpf (rechts) 


\subsection{Herstellung der Kronengerüste}

\subsubsection{Scanprozess}

Bevor der eigentliche CAD/CAM-basierte Herstellungsprozess beginnen konnte, mussten die 20 Meistermodelle digitalisiert werden. In dieser Arbeit wurde dafür der Scanner OpenScan 100 von der Firma Laserdenta (Bergheim) verwendet.

Die Meistermodelle wurden einzeln auf dem Scanteller (Dental-Halter) positioniert, welcher über einen Magneten im Innenraum des Lasers fixiert wurde. Da der Scanner auch voll- oder teilbezahnte Kiefer scannen kann, wurde von der Software abgefragt, welche Situation digitalisiert werden soll. Die Option Einzelscan wurde ausgewählt und die ungefähre Modellhöhe angegeben. Die Software zeigte daraufhin ein Foto des Objekts an, damit die Mitte des Meistermodells per Fadenkreuz bestimmt werden konnte. Nachdem manuell eingegeben worden war, um welchen Zahn es sich handelte, startete der Scanprozess.

Der OpenScan 100 nutzt das Prinzip der Triangulation. Die Strahlrichtung der ausgesandten Laserlinie und der Abstand zwischen Lichtquelle und Kamera sind bekannt, sodass aus der Reflektion der Laserlinie der Abstand von der aufgenommenen Oberfläche zur Kamera bestimmt werden kann. Da diese Berechnung kontinuierlich durchgeführt wird, kann das Oberflächenrelief vollständig digitalisiert werden.

Nach der Kontrolle des Abbilds konnten die Daten in das CAD-System exportiert werden.

\subsubsection{CAD-Prozess}

Die verwendete CAD-Software (OpenCAD V.4) stammt ebenfalls von der Firma Laserdenta (Bergheim).

Um die Präparationsgrenze zu bestimmen, wurden zwei gegenüberliegende Punkte markiert. Anhand dieser beiden Punkte wurde von der Software eine Linie erstellt, die der Präparationsgrenze weitgehend entsprach. Gab es Abweichungen zwischen der Linie und der Präparationsgrenze, wurde die Linie manuell korrigiert. 
Anschließend wurden die Einschubrichtung festgelegt und die vorhandenen Unterschnitte beseitigt.

Die Software kann neben Käppchen auch anatomische Kronen und Inlays, Brückenglieder und Abutments designen. Nachdem als Restaurationsart Käppchen ausgewählt worden war, konnten die Parameter eingestellt werden. Die optimalen Werte wurden in einer Pilotstudie ermittelt.

\subsubsection{Pilotstudie}

In der Pilotstudie sollten die Parameter ermittelt werden, die eine möglichst gute makroskopische Passung der Käppchen bewirken. Die Käppchen sollten nicht durch einen Zahntechniker aufgepasst werden, da dies die Vergleichbarkeit eingeschränkt hätte. Daher durften die Käppchen keine zu hohe Friktion aufweisen.

Die Parameter in der Pilotstudie wurden an Kobalt-Chrom-Käppchen getestet. Im ersten Versuch wurden die Werkseinstellungen übernommen, die einen Zementspalt von $30 \mu \mathrm{m}$ und einen Abstand des Zementspalts von $1 \mathrm{~mm}$ vorgaben. Der erste Wert beschreibt den vorhandenen Raum zwischen Zahnstumpf und Käppchen, der im klinischen Alltag dem Zement beim Einzementieren Platz gibt. Der zweite Wert gibt an, wie weit der Zementspalt von der Präparationsgrenze entfernt ist (siehe Abb. 13)

Da das Versuchskäppchen mit den Werkseinstellungen zu viel Spiel hatte, wurde der Abstand des Zementspalts variiert und später auf 1,5 mm festgelegt.

Nachdem die Parameter für Kobalt-Chrom bestimmt worden waren, konnten Testkäppchen aus Titan, Glaskeramik und Zirkoniumdioxid hergestellt werden. Titan wurde mit den gleichen Parametern hergestellt wie Kobalt-Chrom. Bei Glaskeramik und Zirkoniumdioxid mussten die Wandstärken erhöht werden. Die Parameter für die Innengestaltung wurden ebenfalls von Kobalt-Chrom übernommen.

Da alle Testkäppchen nach der Fertigstellung eine gute makroskopische Passung aufwiesen, konnten diese Parameter für die eigentliche Studie übernommen werden. 


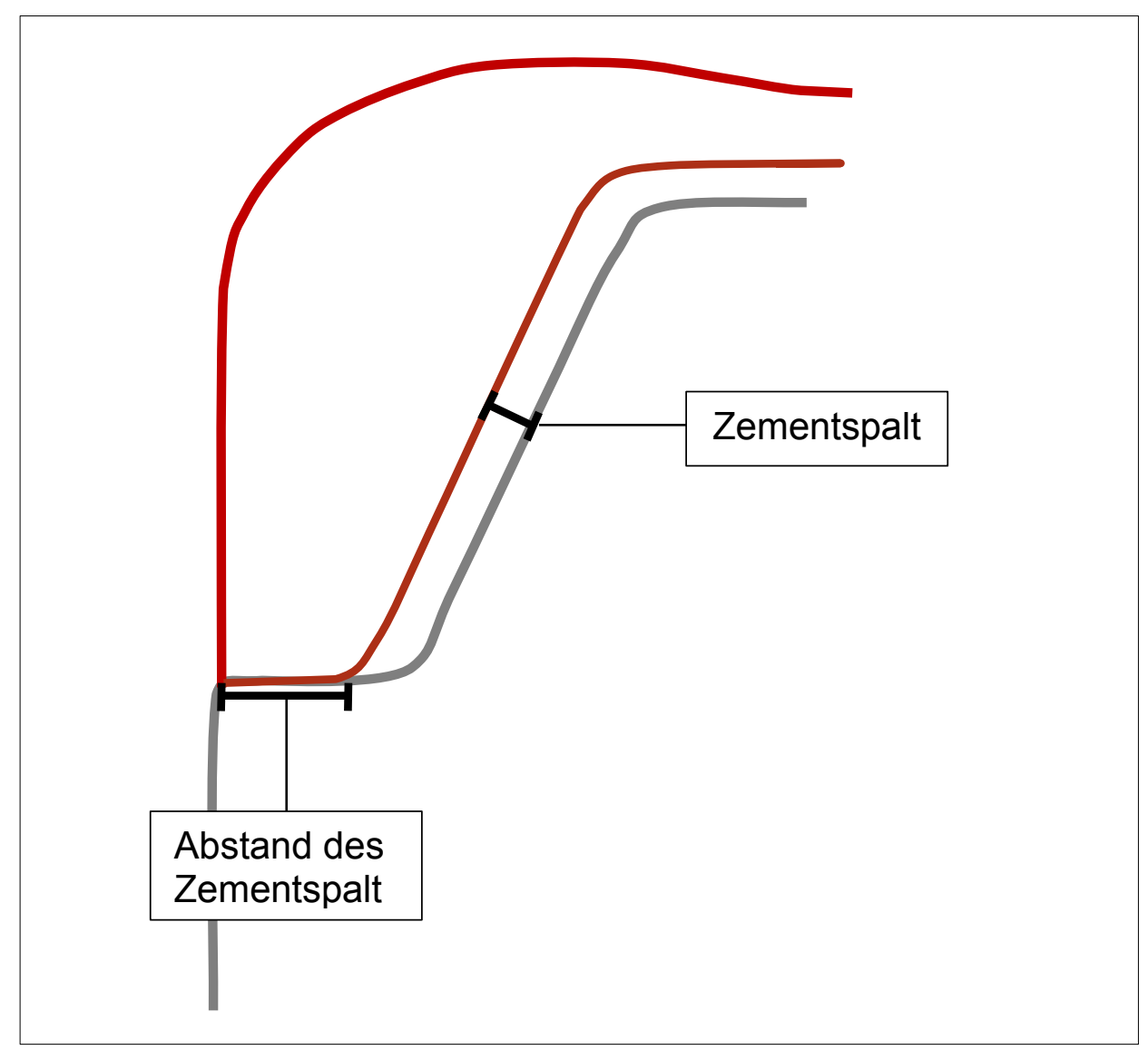

Abbildung 13: Darstellung der Parameter des Innendesigns

\subsubsection{CAM-Prozess}

Die fertiggestellten Käppchendesigns wurden in die DS CAM (Dental Softworks-UG, Wahlsburg) exportiert. Mit dieser Software wurden die Käppchen auf den Rohlingen platziert, die Fräser für die Bearbeitung ausgesucht und die Fräsbahnen berechnet. Diese Daten wurden dann an die Fräsmaschine (GAMMA 202, Wissner, Göttingen) geschickt.

Der Fräsprozess begann mit dem Schruppen der Ober- und Unterseite. Der Schruppvorgang diente der groben Bearbeitung des Werkstücks. Binnen kurzer Zeit sollte so das Werkstück der Endkontur angenähert werden.

Auf das Schruppen folgte der Schlichtvorgang. Dabei wurde die endgültige Form des Werkstücks erreicht, sodass in der darauffolgenden Feinbearbeitung nur noch die Oberfläche geglättet werden musste. 


\subsection{Unterschiede bei der Herstellung}

Um die Vergleichbarkeit zu verbessern und Fehlerquellen zu vermeiden bzw. zu lokalisieren, wurden die Versuche in Testreihen durchgeführt. Eine Testreihe bestand jeweils aus einem Käppchen aus Titan, Kobalt-Chrom, Zirkoniumdioxid und Glaskeramik, die aus derselben Abformung des Masterstumpfes hervorgingen. Pro Abformung entstand ein Meistermodell, welches jeweils genau einmal eingescannt wurde. Nachdem für jeden Stumpf die Einschubrichtung und die Präparationsgrenze bestimmt worden waren, mussten auch diese für die weiteren Materialien nicht erneut festgelegt werden. Als Parameter für die Gerüstinnenfläche wurden jedes Mal die Werte eingestellt, die bei der Pilotstudie ermittelt wurden.

Die Käppchen aus Titan und Kobalt-Chrom wurden mit denselben Datensätzen in die DS CAM-Software exportiert. Bei den Designs für die Keramiken wurden nur die Wandstärken erhöht. Danach wurden auch für Glaskeramik und Zirkoniumdioxid äquivalente Datensätze zur DS CAM-Software geschickt, sodass sich das Innendesign der Kronengerüste innerhalb der Testreihen nicht unterschied.

In der DS CAM-Software wurden jeweils die entsprechenden Fräser ausgewählt und die Käppchen unterschiedlich auf den Rohlingen platziert. Die Sinterschwindung von Zirkoniumdioxid wurde eingegeben, sodass die Software das Design um diesen Betrag vergrößerte.

Titan, Kobalt-Chrom und Zirkoniumdioxid wurden im 3-Achssystem gefräst, Glaskeramik wurde geschliffen und im 5-Achssystem bearbeitet, sodass Unterschiede in den Fräsbahnen entstanden.

Die Bearbeitung von Titan und Kobalt-Chrom erfolgte unter Schwallkühlung während Zirkoniumdioxid trocken gefräst wurde.

Für die einzelnen Schritte der Maschine wurden unterschiedliche Fräsergrößen genutzt. Beim Schruppen wurden für Kobalt-Chrom und Titan Fräser mit $3 \mathrm{~mm}$ Durchmesser, für Zirkoniumdioxid Fräser mit $2 \mathrm{~mm}$ Durchmesser verwendet. Bei Kobalt-Chrom und Titan wurde nach dem Schruppen erst mit einem 2 mm-Fräser, danach mit einem 1,5 mm-Fräser die Innenseite der Käppchen vorgeschlichtet, diese beiden Schritte waren bei Zirkoniumdioxid nicht nötig. 
Die Feinbearbeitung der Innenseite wurde jeweils mit einem $1 \mathrm{~mm}$-Fräser durchgeführt.

Die Feinbearbeitung der Außenfläche erfolgte bei Kobalt-Chrom und Titan erst mit $2 \mathrm{~mm}$ - dann mit 1,5 mm-Fräsern, bei Zirkoniumdioxid mit $1 \mathrm{~mm}$-Fräsern.

Nach der Feinbearbeitung wurden in die Käppchen aus Titan, Kobalt-Chrom und Zirkoniumdioxid Zahlen eingraviert, damit sie später besser den Testreihen zugeordnet werden konnten.

Zum Schluss wurden bei Kobalt-Chrom und Titan die Haltestifte, welche die Käppchen während der Bearbeitung stabilisiert hatten, mit einem $2 \mathrm{~mm}$-Fräser zu $90 \%$ durchtrennt.

Nach der maschinellen Bearbeitung wurden die Restaurationen vollständig aus den Rohlingen gelöst, was bei Titan und Kobalt-Chrom durch Druck auf die Käppchen geschah. Die Haltestifte von Zirkoniumdioxid wurden manuell mit einem HartmetallFräser entfernt.

Die Glaskeramik-Rohlinge wurden nicht gefräst, sondern unter Schwallkühlung geschliffen. Bei der Bearbeitung im 3-Achssystem wurde vor allem mit der Spitze der Schleifwerkzeuge gearbeitet und so von oben nach unten gearbeitet. Bei der Glaskeramikbearbeitung wurde das 5-Achssystem verwendet, bei dem der ganze Umfang des Werkzeugs genutzt wurde. Daher wurde der Rohling hier von außen nach innen beschliffen.

Zuerst wurde die Unterseite des Rohlings bearbeitet. Das Schruppen mit dem 2 mmSchleifkörper legte die grobe Form fest. Daraufhin folgte die Innenbearbeitung. Dabei schliff ein $2 \mathrm{~mm}$-Werkzeug spiralartig in die Tiefe, um die Kavität mit seinem Umfang ausarbeiten zu können. Die Bearbeitung von unten wurde durch das Schlichten mit einem $1 \mathrm{~mm}$-Schleifer beendet. Daraufhin wurde der Rohling gedreht und die Gestaltung der Oberseite konnte in der gleichen Weise stattfinden.

Die Haltestifte der Glaskeramik-Käppchen wurden unter Wasserkühlung mit einem Diamantschleifer manuell durchtrennt. 
Im Anschluss an die Glättung des Übergangs von Käppchen zu Haltestift wurden die Zirkoniumdioxid-Käppchen bei einer Maximaltemperatur von $1350^{\circ} \mathrm{C}$ acht Stunden gesintert.

Die Sinterung der Glaskeramik-Käppchen wurde unter Vakuum bei einer Höchsttemperatur von $850^{\circ} \mathrm{C}$ durchgeführt.

\subsection{Ausschluss von Testreihen}

Trotz der erfolgreichen Pilotstudie fiel nach der Fertigstellung der Kronenkäppchen auf, dass einzelne Käppchen wegen einer ungenügenden Innenpassung nicht an die Präparationsgrenze heranreichten. Da die Kronengerüste nicht nachträglich bearbeitet werden sollten, konnten diese Käppchen nicht in die Studie mit einbezogen werden. Um die Vergleichbarkeit zwischen den Materialien aufrechtzuerhalten, wurden alle Käppchen aus den Testreihen (Bsp.: alle Käppchen Nr. 3), in denen es zu einer makroskopisch mangelhaften Passung eines Käppchens gekommen war, nicht vermessen.

\subsection{Messungen}

Zur Beurteilung der Randpassung wurden Fotos des Randspalts aufgenommen. Dazu wurde der Umfang des Masterstumpfs bestimmt und in 24 gleichgroße Abschnitte unterteilt, sodass 24 Messpunkte im Abstand von ca. $1 \mathrm{~mm}$ entstanden.

Die insgesamt 960 Aufnahmen wurden mit 35facher Vergrößerung mit einem Leica EZ4 D Auflichtmikroskop (Leica-Microsystems, Wetzlar) mit der integrierten Kamera durchgeführt.

Um den von Holmes et al. (1989) als Randschluss definierten Abstand zu messen und keine Verfälschungen durch über- oder unterextendierte Kronenränder zu erhalten, mussten die Fotos im $90^{\circ}$-Winkel zum Spalt aufgenommen werden. Hierfür wurde der Masterstumpf in ein Gerät eingebracht (siehe Abb. 14). Dieses konnte den Winkel zum Randschluss in der Abbildungsachse um bis zu $30^{\circ}$ variieren. 
Die Apparatur verfügte außerdem über eine Rotationsachse, mit der vor der Aufnahme der relevante Abschnitt des Randspalts in das Abbildungszentrum der Kamera gebracht wurde. Damit keine Ungenauigkeiten der Ergebnisse durch unterschiedlichen Anpressdruck auf die Käppchen entstehen konnten, wurde dem Gerät ein Fixationssystem hinzugefügt, das die Käppchen mit gleichmäßigem Druck auf dem Masterstumpf befestigte.

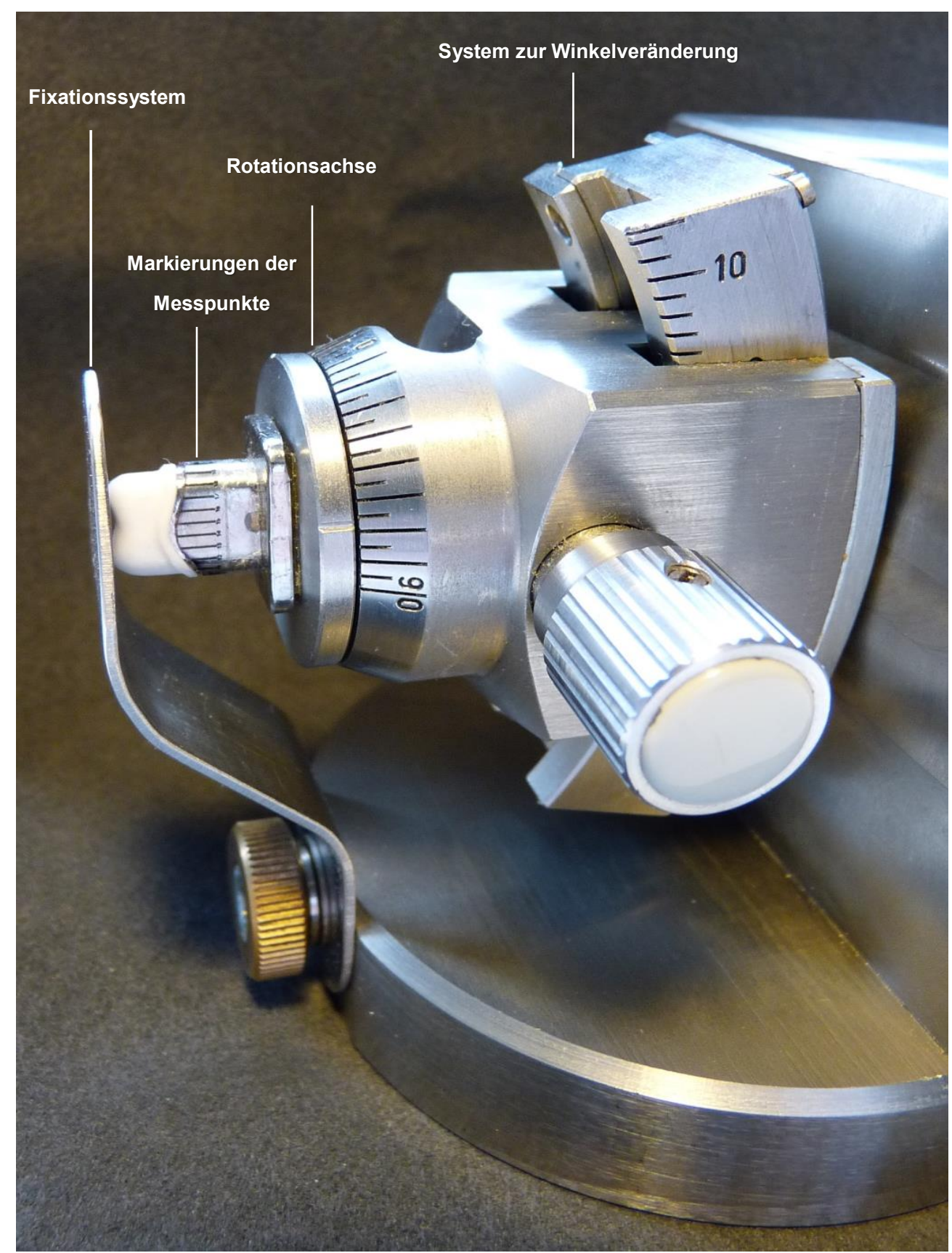

Abb. 14: Gerät zur Aufnahme des Masterstumpfes mit Kronengerüst 
Das momentane Bild durch das Auflichtmikroskop wurde per Software Leica Acquire 1.0 (Leica-Microsystems, Wetzlar) auf dem Computer sichtbar gemacht. Durch Betätigen des Capture-Buttons der Software wurden die Fotos aufgenommen und gespeichert (siehe Abb. 15-18).

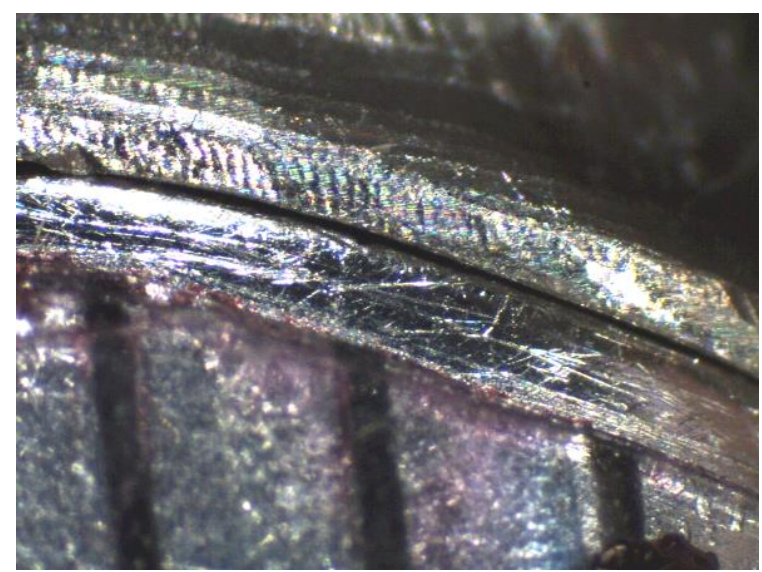

Abb. 15: Randspalt Aufnahme eines Titan-Käppchens

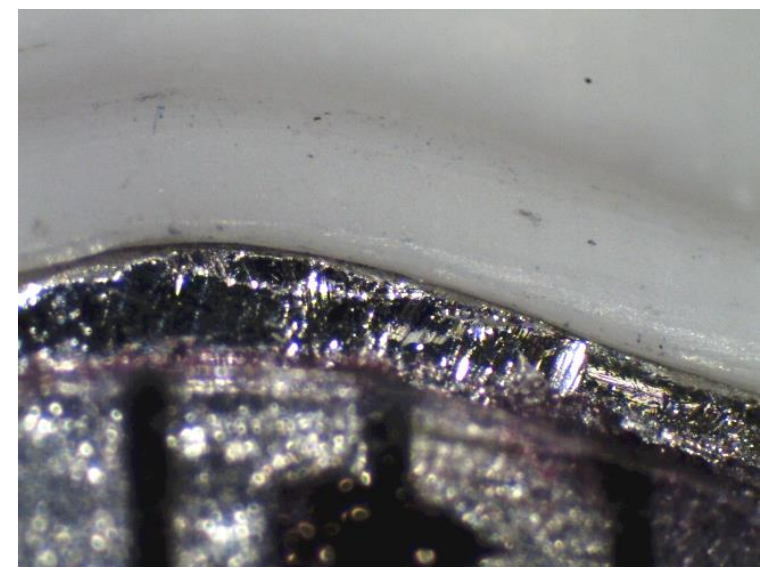

Abb. 17: Randspalt Aufnahme eines

Zirkoniumdioxid-Käppchens

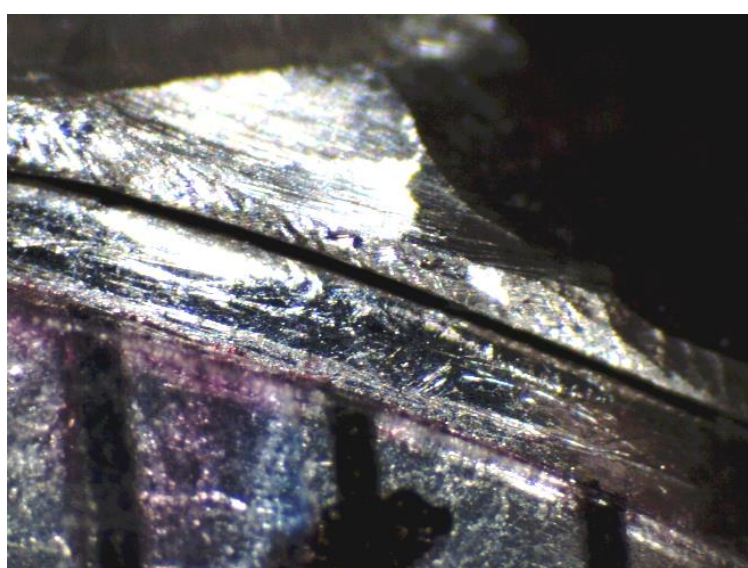

Abb. 16: Randspalt Aufnahme eines Kobalt-Chrom-Käppchens

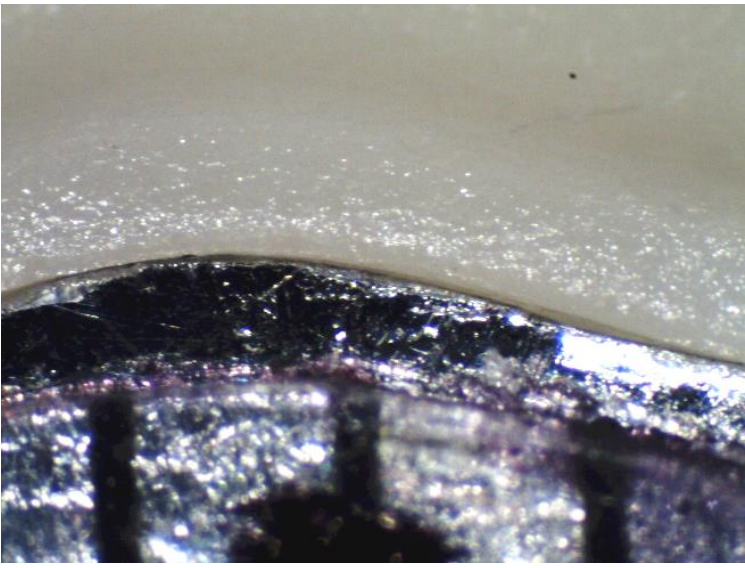

Abb. 18: Randspalt Aufnahme eines Glaskeramik-Käppchens

Mit dem Messwerkzeug der Adobe Photoshop CS5 Software (Adobe Systems Incorporated, San José, USA) wurde der Randspalt auf den Bildern im $90^{\circ}$-Winkel zur Präparationsgrenze vermessen. Das Messwerkzeug zählt die Pixel zwischen den manuell gesetzten Bezugspunkten und errechnet den entsprechenden Abstand in $\mu \mathrm{m}$. Dafür musste mittels Probeaufnahmen eines Messkörpers (Motic Calibration Slide, Motic Deutschland $\mathrm{GmbH}$, Wetzlar) das Messwerkzeug und die MikroskopKamera-Computer-Einheit kalibriert werden. Diese Probeaufnahmen wurden zur Reproduzierbarkeit archiviert. 


\subsection{Statistische Methoden}

Im Rahmen der statistischen Analyse wurde für jedes Käppchen jeweils der Mittelwert und das Maximum der Randspaltmessungen ermittelt und statistisch ausgewertet.

Da auf jedem Gipsstumpf jeweils ein Käppchen aus den verschiedenen Materialien hergestellt wurde, lag aus statistischer Sicht ein einfaktorielles repeated measuresDesign mit dem Faktor Material und den Faktorstufen Titan, Kobalt-Chrom, Zirkoniumdioxid und Glaskeramik vor. Die abhängigen Variablen waren die Mittelwerte und die jeweiligen Maxima der verschiedenen Messpunkte der 10 Käppchen eines Materials. Somit lagen für jedes Material 10 Mittelwerte und 10 Maxima für die Berechnungen signifikanter Unterschiede vor. Als Signifikanzniveau wurde $\alpha=0,05$ gewählt.

Die Nullhypothese lautete: Die Gerüste aller Materialien weisen den gleichen mittleren/maximalen Randspaltwert auf.

Nach dem Test der Nullhypothese wurden multiple Vergleiche der Faktorstufen durchgeführt. Die p-Werte der jeweiligen Paarvergleiche wurden mit Hilfe der Bonferroni-Holm Methode adjustiert.

Die statistische Auswertung wurde in Zusammenarbeit mit dem Institut für Medizinische Statistik der Universitätsmedizin Göttingen durchgeführt. Zur Auswertung der Daten wurde das Statistikprogramm SAS Version 9.3 genutzt (SAS Institute Inc., Cary, NC, USA). 


\section{Ergebnisse}

\subsection{Statistische Auswertung der Randspaltmessungen}

Die Nullhypothese (die Gerüste aller Materialien weisen den gleichen mittleren/maximalen Randspaltwert auf) wurde für die Mittelwerte verworfen $(p=0,0009)$. Das bedeutet, dass zwischen den Materialien ein signifikanter Unterschied für die mittleren Randspaltgrößen der Käppchen bestand.

Die multiplen Vergleiche der Faktorstufen ergaben signifikante Unterschiede für den jeweiligen Vergleich Titan/Zirkoniumdioxid $\quad\left(p_{\text {adj }}=0,0024\right)$, Titan/Glaskeramik $\left(p_{a d j}=0,008\right)$ und Kobalt-Chrom/Zirkoniumdioxid $\left(p_{a d j}=0,0332\right)$.

Ein signifikanter Unterschied zwischen den Materialien bezüglich der maximalen Randspaltwerte der Käppchen lag nicht vor $(p=0,1673)$. Hier wiesen demnach alle untersuchten Materialien vergleichbare maximale Randspaltweiten auf. 


\subsection{Marginale Präzision bei der Verwendung von Titan}

Bei zehn Kronengerüsten aus Titan wurde jeweils an 24 Positionen der Randspalt gemessen, sodass 240 Messwerte entstanden (siehe Tab. 1). Der Mittelwert des Randspalts aller Messpunkte betrug für Titan 46,92 $\mu \mathrm{m}$ bei einer Standardabweichung (Std. Abw. von 23,12 $\mu \mathrm{m}$. Der Maximalwert wurde an Käppchen Nummer 5 gemessen und lag mit 143,71 $\mu$ m oberhalb der geforderten klinischen Toleranzgrenze von $120 \mu \mathrm{m}$. Der Mittelwert der Maxima der Kronenkäppchen betrug für Titan 91,54 $\mu \mathrm{m}$.

Der niedrigste Mittelwert eines Käppchens ergab 37,53 $\mu \mathrm{m}$ und wurde bei Testreihe Nummer 12 ermittelt. Das Kronengerüst mit der schlechtesten mittleren Passung war auch diesbezüglich Käppchen Nummer 5. Es hatte einen Mittelwert von 62,77 $\mu \mathrm{m}$. 


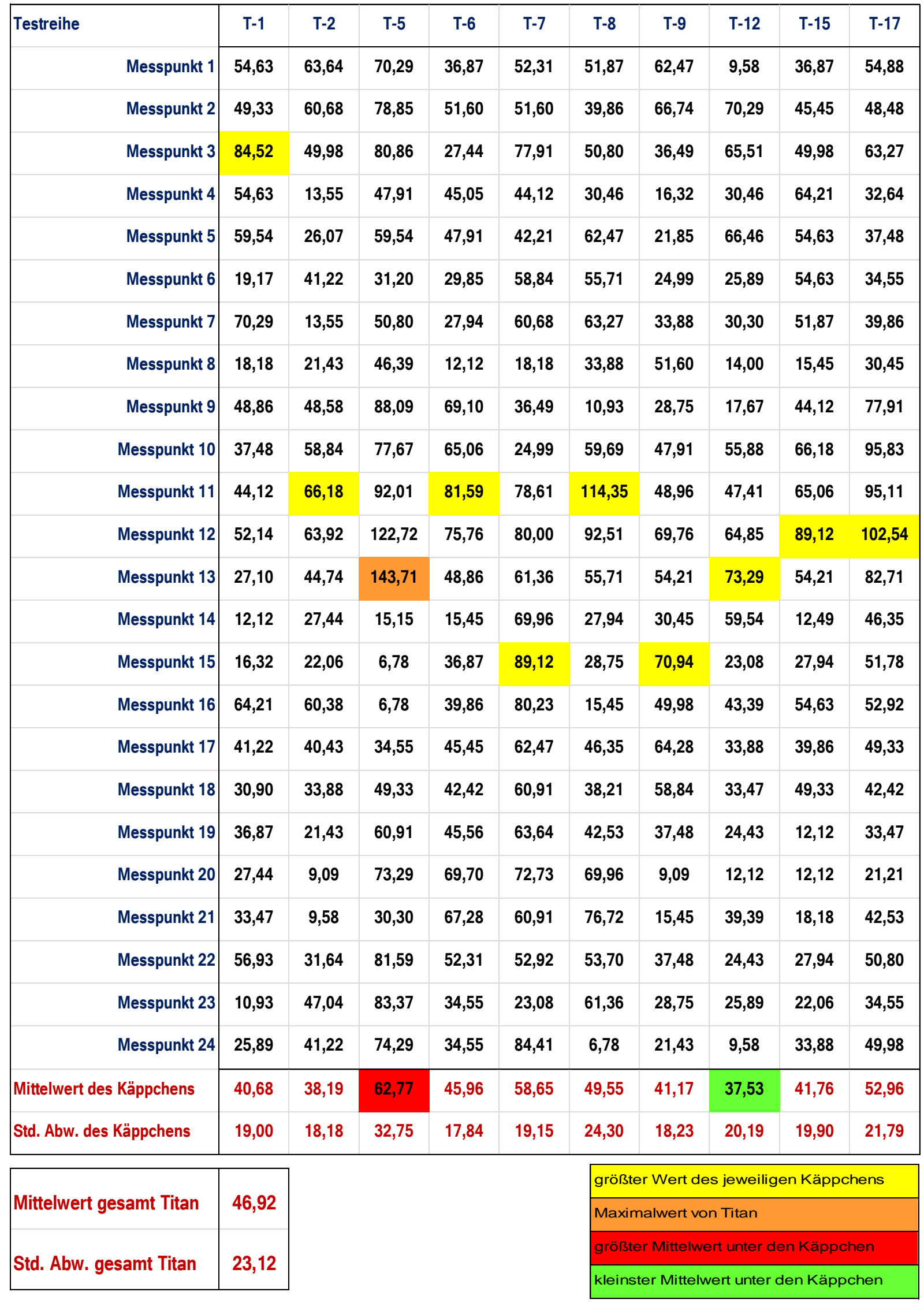

Tab. 1: Marginale Präzision bei der Verwendung von Titan 


\subsection{Marginale Präzision bei der Verwendung von Kobalt-Chrom}

Der Mittelwert der 240 Randspaltmessungen an den zehn Kobalt-Chrom-Käppchen ergab 48,37 $\mu \mathrm{m}$ bei einer Standardabweichung von 29,72 $\mu \mathrm{m}$ (siehe Tab. 2). Der größte gemessene Wert lag mit 156,44 $\mu$ m nicht mehr im klinischen Toleranzbereich. Wie bei Titan wurde der Maximalwert an Käppchen Nummer 5 festgestellt. Der gemittelte Maximalwert aller Käppchen betrug 96,86 $\mu \mathrm{m}$.

Mit 31,11 $\mu \mathrm{m}$ hatte Käppchen Nummer 2 die beste mittlere Passung. Ebenfalls wie bei Titan wies das Kronengerüst Nummer 5 auch die schlechteste mittlere Passung auf. Der Mittelwert des Käppchens Nummer 5 betrug 94,57 $\mu \mathrm{m}$. 


\begin{tabular}{|c|c|c|c|c|c|c|c|c|c|c|}
\hline Testreihe & C-1 & C-2 & C-5 & C-6 & C-7 & C-8 & C-9 & C-12 & C-15 & C-17 \\
\hline Messpunkt 1 & 27,94 & 45,56 & 115,79 & 28,75 & 40,43 & 91,71 & 15,45 & 36,87 & 52,31 & 21,43 \\
\hline Messpunkt 2 & 51,60 & 39,39 & 110,14 & 39,86 & 43,39 & 103,03 & 12,12 & 63,71 & 54,63 & 21,21 \\
\hline Messpunkt 3 & 33,88 & 13,55 & 116,97 & 23,08 & 45,05 & 62,47 & 12,49 & 27,94 & 58,52 & 28,75 \\
\hline Messpunkt 4 & 31,64 & 13,55 & 87,25 & 37,97 & 44,12 & 38,33 & 6,06 & 98,71 & 56,93 & 15,45 \\
\hline Messpunkt 5 & 15,45 & 9,58 & 84,20 & 33,88 & 31,64 & 47,91 & 41,22 & 77,67 & 32,64 & 35,47 \\
\hline Messpunkt 6 & 24,99 & 9,58 & 63,27 & 43,39 & 21,43 & 64,21 & 15,45 & 49,33 & 42,21 & 24,99 \\
\hline Messpunkt 7 & 72,79 & 22,06 & 88,09 & 31,64 & 46,35 & 45,86 & 33,47 & 21,21 & 47,04 & 51,87 \\
\hline Messpunkt 8 & 66,94 & 15,45 & 101,64 & 12,12 & 6,06 & 18,43 & 64,28 & 22,06 & 15,15 & 33,33 \\
\hline Messpunkt 9 & 82,32 & 57,89 & 126,55 & 48,86 & 18,18 & 6,78 & 67,76 & 30,90 & 45,86 & 71,32 \\
\hline Messpunkt 10 & 69,96 & 75,76 & 147,77 & 59,69 & 31,20 & 57,50 & 78,79 & 63,92 & 75,76 & 69,96 \\
\hline Messpunkt 11 & 80,06 & 47,43 & 109,26 & 68,10 & 85,76 & 75,76 & 67,28 & 62,40 & 81,31 & 65,06 \\
\hline Messpunkt 12 & 39,39 & 47,14 & 154,55 & 69,76 & 83,21 & 51,52 & 76,48 & 63,92 & 103,43 & 76,48 \\
\hline Messpunkt 13 & 40,66 & 15,15 & 9,58 & 68,10 & 84,41 & 17,67 & 66,46 & 58,84 & 21,63 & 20,33 \\
\hline Messpunkt 14 & 12,12 & 18,43 & 12,49 & 18,18 & 72,98 & 6,06 & 63,27 & 12,49 & 6,78 & 51,87 \\
\hline Messpunkt 15 & 24,99 & 27,94 & 12,12 & 41,22 & 40,43 & 18,43 & 105,19 & 9,09 & 54,63 & 47,91 \\
\hline Messpunkt 16 & 90,10 & 41,22 & 22,06 & 42,21 & 63,27 & 15,45 & 67,28 & 55,88 & 58,84 & 53,70 \\
\hline Messpunkt 17 & 77,26 & 25,89 & 70,74 & 55,71 & 59,54 & 46,35 & 67,28 & 48,86 & 49,33 & 39,86 \\
\hline Messpunkt 18 & 48,86 & 33,88 & 88,09 & 45,86 & 55,30 & 48,48 & 66,94 & 33,88 & 33,47 & 39,86 \\
\hline Messpunkt 19 & 48,58 & 30,30 & 118,18 & 48,86 & 60,91 & 24,24 & 45,45 & 27,27 & 36,36 & 30,30 \\
\hline Messpunkt 20 & 36,36 & 18,18 & 118,22 & 72,73 & 81,82 & 45,45 & 12,12 & 6,06 & 12,49 & 21,21 \\
\hline Messpunkt 21 & 36,49 & 6,78 & 101,14 & 73,73 & 60,91 & 42,53 & 30,90 & 36,49 & 30,30 & 55,30 \\
\hline Messpunkt 22 & 38,33 & 50,80 & 146,62 & 40,43 & 41,22 & 51,78 & 49,33 & 24,99 & 34,55 & 72,03 \\
\hline Messpunkt 23 & 25,89 & 53,70 & 156,44 & 43,39 & 12,49 & 43,39 & 41,22 & 47,04 & 24,99 & 17,67 \\
\hline Messpunkt 24 & 28,75 & 27,44 & 108,42 & 12,49 & 16,32 & 6,78 & 37,48 & 6,78 & 71,32 & 53,70 \\
\hline Mittelwert des Käppchens & 46,06 & 31,11 & 94,57 & 44,17 & 47,77 & 42,92 & 47,66 & 41,10 & 45,85 & 42,46 \\
\hline Std. Abw. des Käppchens & 22,21 & 17,81 & 43,11 & 17,85 & 23,17 & 25,24 & 25,95 & 23,44 & 22,53 & 19,06 \\
\hline
\end{tabular}

\begin{tabular}{|l|l|}
\hline Mittelwert gesamt CoCr & 48,37 \\
\hline Std. Abw. gesamt CoCr & 29,72 \\
\hline
\end{tabular}

\begin{tabular}{|l|}
\hline größter Wert des jeweiligen Käppchens \\
\hline Maximalwert von Kobalt-Chrom \\
\hline größter Mittelwert unter den Käppchen \\
\hline kleinster Mittelwert unter den Käppchen \\
\hline
\end{tabular}

Tab. 2: Marginale Präzision bei der Verwendung von Kobalt-Chrom 


\subsection{Marginale Präzision bei der Verwendung von Zirkoniumdioxid}

Der Mittelwert für die 240 Messpunkte von Zirkoniumdioxid betrug 68,25 $\mu \mathrm{m}$ bei einer Standardabweichung von 28,54 $\mu \mathrm{m}$ (siehe Tab. 3).

Der höchste Wert wurde wiederum an Kronengerüst Nummer 5 festgestellt und betrug 183,15 $\mu \mathrm{m}$. Damit liegt er weit oberhalb der klinischen Toleranzgrenze. Bei den Zirkoniumdioxid-Käppchen ergab der Mittelwert der Maxima 120,66 $\mu \mathrm{m}$ und liegt damit nur knapp über der klinischen Toleranzgrenze.

Auch bei Zirkoniumdioxid wies Käppchen Nummer 5 mit 97,20 $\mu \mathrm{m}$ die schlechteste mittlere Passung auf. Käppchen Nummer 17 hatte mit 48,74 $\mu \mathrm{m}$ die beste mittlere Passung. 


\begin{tabular}{|c|c|c|c|c|c|c|c|c|c|c|}
\hline Testreihe & $Z-1$ & Z-2 & Z-5 & Z-6 & Z-7 & Z-8 & Z-9 & Z-12 & Z-15 & Z-17 \\
\hline Messpunkt 1 & 72,79 & 68,37 & 140,87 & 51,87 & 67,76 & 89,18 & 73,73 & 52,31 & 39,86 & 42,85 \\
\hline Messpunkt 2 & 73,29 & 39,51 & 112,16 & 64,78 & 81,87 & 72,73 & 81,87 & 76,30 & 54,63 & 45,56 \\
\hline Messpunkt 3 & 95,83 & 39,86 & 117,09 & 83,37 & 95,68 & 126,04 & 130,16 & 65,42 & 61,81 & 72,03 \\
\hline Messpunkt 4 & 116,62 & 81,59 & 183,15 & 66,46 & 75,76 & 104,49 & 121,97 & 81,59 & 60,38 & 64,21 \\
\hline Messpunkt 5 & 116,94 & 140,38 & 137,04 & 97,02 & 90,40 & 98,15 & 128,92 & 66,18 & 61,36 & 68,10 \\
\hline Messpunkt 6 & 116,97 & 119,07 & 132,30 & 90,10 & 74,97 & 91,71 & 91,16 & 109,26 & 59,54 & 40,43 \\
\hline Messpunkt 7 & 81,59 & 82,32 & 95,68 & 55,88 & 55,30 & 52,31 & 67,28 & 48,86 & 43,39 & 36,87 \\
\hline Messpunkt 8 & 100,18 & 84,90 & 72,98 & 72,73 & 72,73 & 70,74 & 90,96 & 54,55 & 28,00 & 24,99 \\
\hline Messpunkt 9 & 88,09 & 53,70 & 111,75 & 36,87 & 39,86 & 47,91 & 73,73 & 33,47 & 46,35 & 34,55 \\
\hline Messpunkt 10 & 69,10 & 110,30 & 145,01 & 65,27 & 74,29 & 58,52 & 76,66 & 33,88 & 77,26 & 63,27 \\
\hline Messpunkt 11 & 77,67 & 108,63 & 122,72 & 78,20 & 131,46 & 68,10 & 92,16 & 126,15 & 58,29 & 66,53 \\
\hline Messpunkt 12 & 43,07 & 69,17 & 131,57 & 62,40 & 92,36 & 62,40 & 59,77 & 70,68 & 67,28 & 71,52 \\
\hline Messpunkt 13 & 36,62 & 146,37 & 59,69 & 54,21 & 104,36 & 69,17 & 42,10 & 78,61 & 54,21 & 43,70 \\
\hline Messpunkt 14 & 21,43 & 46,35 & 56,61 & 38,33 & 112,45 & 50,80 & 30,90 & 46,69 & 40,43 & 36,87 \\
\hline Messpunkt 15 & 31,64 & 48,86 & 36,87 & 48,86 & 80,23 & 40,43 & 50,80 & 63,27 & 40,43 & 49,98 \\
\hline Messpunkt 16 & 45,05 & 41,22 & 42,85 & 42,85 & 33,88 & 34,55 & 35,47 & 100,73 & 52,92 & 22,06 \\
\hline Messpunkt 17 & 61,36 & 48,86 & 58,29 & 64,28 & 39,51 & 90,91 & 43,39 & 72,03 & 40,43 & 33,88 \\
\hline Messpunkt 18 & 58,29 & 51,52 & 48,58 & 68,37 & 66,74 & 54,55 & 73,73 & 55,30 & 51,87 & 39,39 \\
\hline Messpunkt 19 & 24,99 & 51,60 & 57,89 & 75,82 & 97,39 & 78,85 & 66,94 & 48,48 & 48,58 & 51,60 \\
\hline Messpunkt 20 & 35,47 & 57,58 & 64,28 & 60,68 & 97,02 & 71,32 & 66,74 & 36,36 & 30,30 & 36,36 \\
\hline Messpunkt 21 & 33,47 & 36,87 & 51,87 & 64,28 & 73,29 & 61,81 & 63,64 & 39,51 & 27,44 & 45,86 \\
\hline Messpunkt 22 & 52,92 & 69,23 & 128,92 & 65,42 & 82,43 & 96,30 & 99,26 & 22,80 & 50,80 & 61,36 \\
\hline Messpunkt 23 & 52,31 & 45,05 & 127,85 & 39,39 & 42,21 & 63,27 & 74,29 & 41,22 & 35,47 & 54,63 \\
\hline Messpunkt 24 & 91,36 & 61,36 & 96,83 & 47,04 & 83,37 & 62,47 & 89,74 & 59,54 & 61,81 & 63,27 \\
\hline Mittelwert des Käppchens & 66,54 & 70,94 & 97,20 & 62,27 & 77,72 & 71,53 & 76,06 & 61,80 & 49,70 & 48,74 \\
\hline Std. Abw. des Käppchens & 29,25 & 31,50 & 39,69 & 15,56 & 23,46 & 21,43 & 26,24 & 24,53 & 12,58 & 14,41 \\
\hline
\end{tabular}

\begin{tabular}{|l|l|}
\hline Mittelwert gesamt Zirkon & 68,25 \\
\hline Std. Abw. gesamt Zirkon & 28,54 \\
\hline
\end{tabular}

\begin{tabular}{|l|}
\hline größter Wert des jeweiligen Käppchens \\
\hline Maximalwert von Zirkoniumdioxid \\
\hline größter Mittelwert unter den Käppchen \\
\hline kleinster Mittelwert unter den Käppchen \\
\hline
\end{tabular}

Tab. 3: Marginale Präzision bei der Verwendung von Zirkoniumdioxid 


\subsection{Marginale Präzision bei der Verwendung von Glaskeramik}

Der Mittelwert aller gemessenen Randspalten der zehn Glaskeramik-Käppchen betrug 58,73 $\mu \mathrm{m}$ bei einer Standardabweichung von 21,15 $\mu \mathrm{m}$ (siehe Tab. 4).

Der höchste Wert wurde, wie auch bei den anderen Materialien, bei Käppchen Nummer 5 gemessen und betrug 118,03 $\mu \mathrm{m}$. Ebenfalls wurde bei Kronengerüst Nummer 5 mit 67,17 $\mu \mathrm{m}$ der höchste Wert für die mittlere Passung der Gerüste ermittelt. Der gemittelte Maximalwert aller Glaskeramik-Käppchen betrug 100,22 $\mu \mathrm{m}$. Die beste mittlere Passung besaß Käppchen Nummer 17 mit einem Wert von $51,10 \mu \mathrm{m}$. 


\begin{tabular}{|c|c|c|c|c|c|c|c|c|c|c|}
\hline Testreihe & G-1 & G-2 & G-5 & G-6 & G-7 & G-8 & G-9 & G-12 & G-15 & G-17 \\
\hline Messpunkt 1 & 45,86 & 64,28 & 70,74 & 61,81 & 73,73 & 48,58 & 45,45 & 64,28 & 36,87 & 52,92 \\
\hline Messpunkt 2 & 66,94 & 30,30 & 79,31 & 54,55 & 54,55 & 33,47 & 57,89 & 69,70 & 36,49 & 51,52 \\
\hline Messpunkt 3 & 91,36 & 103,65 & 116,14 & 64,78 & 77,26 & 69,96 & 67,08 & 58,84 & 76,66 & 69,10 \\
\hline Messpunkt 4 & 99,95 & 86,24 & 90,10 & 80,52 & 83,37 & 98,71 & 77,67 & 95,83 & 53,70 & 60,38 \\
\hline Messpunkt 5 & 98,15 & 77,67 & 60,38 & 84,52 & 75,76 & 117,05 & 37,48 & 47,04 & 65,27 & 38,33 \\
\hline Messpunkt 6 & 58,84 & 88,35 & 68,10 & 59,54 & 63,27 & 97,02 & 76,66 & 34,55 & 78,67 & 23,08 \\
\hline Messpunkt 7 & 54,88 & 52,92 & 24,24 & 51,87 & 45,86 & 92,71 & 72,03 & 33,88 & 54,88 & 33,33 \\
\hline Messpunkt 8 & 101,14 & 75,82 & 33,88 & 42,53 & 24,24 & 57,66 & 64,28 & 21,21 & 45,56 & 27,44 \\
\hline Messpunkt 9 & 94,38 & 74,97 & 68,37 & 49,33 & 20,33 & 22,06 & 90,96 & 33,88 & 62,47 & 33,47 \\
\hline Messpunkt 10 & 80,52 & 62,47 & 96,69 & 42,21 & 29,85 & 50,16 & 99,68 & 65,27 & 88,09 & 52,92 \\
\hline Messpunkt 11 & 95,11 & 46,16 & 109,30 & 28,75 & 55,71 & 74,84 & 67,28 & 58,52 & 65,27 & 65,06 \\
\hline Messpunkt 12 & 47,43 & 81,54 & 118,03 & 50,16 & 67,28 & 90,96 & 61,36 & 54,63 & 82,43 & 88,35 \\
\hline Messpunkt 13 & 47,91 & 85,39 & 111,26 & 55,63 & 74,84 & 68,10 & 78,79 & 73,29 & 71,84 & 61,36 \\
\hline Messpunkt 14 & 15,15 & 58,84 & 64,78 & 30,90 & 49,33 & 9,58 & 27,44 & 43,39 & 31,64 & 39,51 \\
\hline Messpunkt 15 & 27,94 & 61,28 & 39,86 & 47,04 & 37,48 & 37,48 & 41,22 & 43,39 & 34,55 & 57,89 \\
\hline Messpunkt 16 & 55,30 & 37,48 & 30,45 & 46,35 & 47,91 & 61,81 & 52,31 & 44,12 & 46,35 & 52,31 \\
\hline Messpunkt 17 & 40,43 & 73,29 & 54,63 & 30,45 & 101,64 & 52,31 & 44,12 & 30,90 & 27,44 & 42,85 \\
\hline Messpunkt 18 & 51,87 & 63,92 & 27,44 & 39,51 & 86,19 & 60,91 & 57,89 & 26,08 & 42,85 & 51,52 \\
\hline Messpunkt 19 & 69,76 & 42,53 & 51,60 & 51,60 & 60,91 & 60,61 & 33,33 & 39,39 & 33,33 & 45,45 \\
\hline Messpunkt 20 & 69,76 & 54,55 & 51,52 & 88,09 & 82,71 & 81,82 & 42,85 & 48,48 & 27,44 & 48,48 \\
\hline Messpunkt 21 & 48,86 & 27,94 & 34,55 & 88,71 & 67,28 & 67,28 & 39,39 & 55,88 & 46,35 & 48,86 \\
\hline Messpunkt 22 & 67,08 & 58,52 & 68,10 & 50,80 & 64,78 & 90,10 & 55,30 & 67,08 & 52,92 & 61,36 \\
\hline Messpunkt 23 & 51,78 & 66,18 & 72,03 & 38,33 & 41,22 & 63,27 & 56,61 & 57,50 & 46,16 & 62,47 \\
\hline Messpunkt 24 & 38,33 & 54,63 & 70,48 & 47,91 & 67,76 & 49,98 & 58,29 & 84,52 & 49,33 & 58,52 \\
\hline Mittelwert des Käppchens & 63,28 & 63,71 & 67,17 & 53,58 & 60,55 & 64,85 & 58,56 & 52,15 & 52,36 & 51,10 \\
\hline Std. Abw. des Käppchens & 23,54 & 18,54 & 27,90 & 16,91 & 20,17 & 25,01 & 17,81 & 18,11 & 17,54 & 14,18 \\
\hline
\end{tabular}

\begin{tabular}{|l|l|}
\hline Mittelwert gesamt Glaskeramik & 58,73 \\
\hline Std. Abw. gesamt Glaskeramik & 21,15 \\
\hline
\end{tabular}

\begin{tabular}{|l|}
\hline größter Wert des jeweiligen Käppchens \\
\hline Maximalwert von Glaskeramik \\
\hline größter Mittelwert unter den Käppchen \\
\hline kleinster Mittelwert unter den Käppchen \\
\hline
\end{tabular}

Tab. 4: Marginale Präzision bei der Verwendung von Glaskeramik 


\subsection{Grafische Darstellung der Ergebnisse}

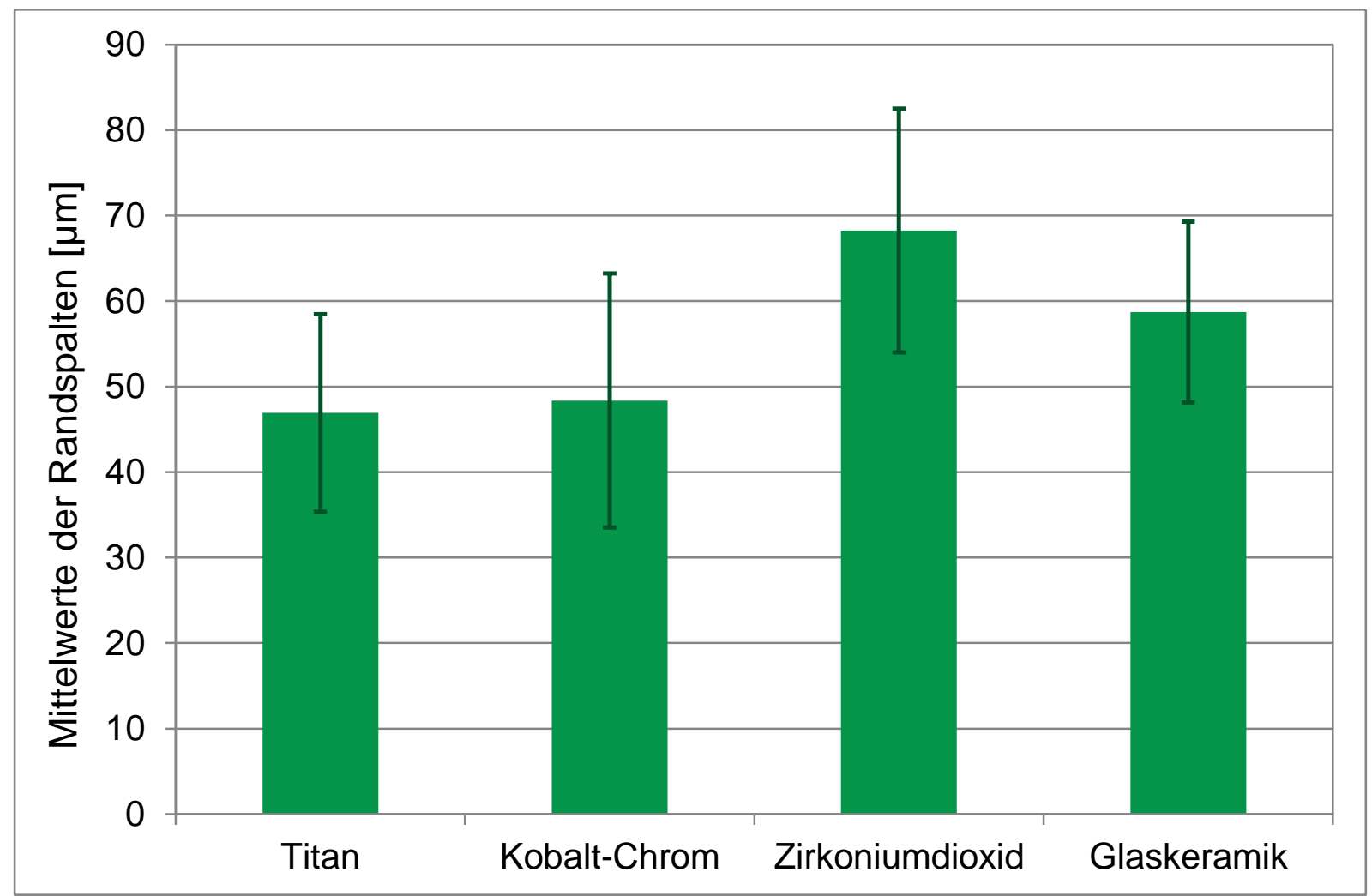

Abb. 19: Vergleich der Passungsgenauigkeiten der unterschiedlichen Materialien mit Angabe der Standardabweichung

Abbildung 19 verdeutlicht die unterschiedlichen mittleren Passungen und die Standardabweichung der verwendeten Materialien. 


\section{Diskussion}

\subsection{Beurteilung der Ergebnisse}

In der vorliegenden Arbeit wurde die In-vitro-Methode zur Untersuchung der Randspalten von CAD/CAM-gefertigten Kronengerüsten aus verschiedenen dentalen Werkstoffen gewählt. Ein Vorteil der in-vitro-Methode ist die Umgehung von Problemen wie eine mangelnde Patientencompliance oder erschwertes Arbeiten im feuchten Mundmilieu unter beeinträchtigter Sicht. Es mussten keine individuellen Präparationen angefertigt werden, wie es bei klinischen Studien unumgänglich ist. Alle Kronenkäppchen wurden auf demselben Masterstumpf aufgepasst, sodass die Ergebnisse in Bezug auf die vorgenommenen Messungen vergleichbar sind.

Trotzdem wurde versucht, die Laborstudie unter möglichst kliniknahen Bedingungen (Herstellungsprozedere) durchzuführen, um die Ergebnisse für klinische Situationen nutzen zu können.

Der Masterstumpf wurde aus einer Metalllegierung hergestellt, sodass eine ausreichende Formstabilität während der Abformungen, dem Aufsetzen der Käppchen und während der Messungen gewährleistet war. Da der Masterstumpf für eine vorhergehende Arbeit zur Untersuchung von Zirkoniumdioxid-Käppchen erstellt wurde (Fornefett 2010), hielt sich die Präparation an die Richtlinien für Vollkeramikkronen. Als Präparationsgrenze wurde eine nach innen abgerundete zirkuläre Stufe gewählt, da Vollkeramikkronen bei diesem Präparationsdesign die größte Belastbarkeit zeigen (Funk 2007). Der Konuswinkel wurde mit $4^{\circ}$ relativ klein gewählt, da ein gößerer Konuswinkel im klinischen Altag zu einem erhöhten Dezementierungsrisiko führt. Je größer der Konuswinkel ist, desto geringer ist außerdem die Retention der Krone (Hajtó 2010).

Obwohl in vergleichenden Studien größere Konvergenzwinkel eine bessere marginale Passung zeigten, war die marginale Passung der Kronen bei $4^{\circ}$ Konuswinkel jeweils im klinisch geeigneten Bereich (Fick 2003; Fürst 2008). 
Im klinischen Alltag sind Abweichungen bei der Abformung und bei dem Ausgießen nicht zu vermeiden (Noack 2012). Um dies in der vorliegenden Studie nachzuempfinden, wurden von dem Metallstumpf 20 Abformungen mit einem Polyether (Impregum Penta, 3M ESPE AG, Seefeld) hergestellt, die mit Superhartgips (GC FujiRock EP, GC Europe N.V., Leuven, Belgien) ausgegossen wurden. Diese Vorgehensweise erzeugte individuelle Gipsmodelle, welche die Passungen in der Studie analog zur Praxis beeinflusste.

$\mathrm{Da}$ verschiedene CAD/CAM-Systeme ebenfalls in unterschiedlichem Maße die Größe des Randspalts beeinflussen (Hamza et al. 2013; Lachmann 2010), wurde in dieser Studie zur Herstellung der Kronengerüste für alle verwendeten Materialien nur ein CAD/CAM-System verwendet.

Das Abformen des Masterstumpfes, das Ausgießen der Abformungen zur Erstellung der Meistermodelle, das Einscannen der Gipsmodelle, die Bestimmung der Präparationsgrenze und die Bestimmung der Einschubrichtung wurde für die unterschiedlichen Käppchen einer Testreihe genau einmal durchgeführt und damit innerhalb einer Testreihe konstant gehalten. Da auch dieselben Werte für das Design der Gerüstinnenfläche verwendet wurden, wiesen die Kronengerüste einer Testreihe ein identisches Innendesign auf.

Um geeignete Werte für das Design der Gerüstinnenfläche zu ermitteln, wurde eine Pilotstudie durchgeführt. Dabei wurden sieben Kobalt-Chrom-Käppchen mit unterschiedlichen Parametern für den Zementspalt und den Abstand des Zementspalts von der Präparationsgrenze hergestellt (siehe Abb. 13). Die Käppchen sollten mit wenig Friktion auf den Masterstumpf aufgesetzt werden können ohne in der Sollposition zu rotieren. Als geeignete Werte für Kobalt-Chrom wurden $30 \mu \mathrm{m}$ Zementspalt und 1,5 mm Abstand des Zementspalts ermittelt. Mit denselben Werten für die Gerüstinnenfläche wurde jeweils ein Testkäppchen aus Titan, Zirkon und Glaskeramik angefertigt. Da diese Kronengerüste nach dem Fräsen bzw. nach dem Sintern ebenfalls eine geeignete Passung aufwiesen, wurden diese Werte für die eigentliche Studie übernommen. 
Trotz der erfolgreichen Pilotstudie kam es nach Fertigstellung aller Käppchen bei einigen Kronengerüsten zu ungenügenden Innenpassungen, sodass sie nicht in Sollposition gebracht werden konnten. Die Käppchen sollten nicht nachträglich bearbeitet werden, da ein zusätzliches Aufpassen den marginalen Randschluss verändert (Fick 2003; Fornefett 2010; Witkowski et al. 2006) und die Vergleichbarkeit der Gerüste einschränkt.

Durch das Beibehalten der Gruppierung konnte festgestellt werden, dass in einigen Testreihen alle Kronengerüste eine ungenügende Passung hatten. In anderen Testreihen konnten nur einzelne Käppchen nicht in Sollposition überführt werden. Um die Vergleichbarkeit der Ergebnisse trotzdem zu gewährleisten, wurden alle Käppchen einer Testreihe, in der es zu einer makroskopisch fehlerhaften Passung kam, nicht vermessen und nicht weiter ausgewertet.

Dies betraf die Testreihen Nummer 3, 4, 10, 11, 13, 14, 16, 18 und 19. Zur Fehlerlokalisation wurden alle Käppchen dieser Testreihen erneut auf die zugehörigen Gipsmodelle gesetzt und mit ihrer Passung auf dem Masterstumpf verglichen.

In den Reihen Nummer 10 und 18 hatten einige der Kronengerüste, die auf dem Mastermodell eine ungenügende Passung hatten, auf dem Meistermodell eine ausreichende Innenpassung. Dies lässt darauf schließen, dass es bei den Abformungen 10 und 18 zu größeren Abweichungen gekommen ist.

Die vom Hersteller empfohlene Abbindezeit von fünf Minuten wurde befolgt und die Rückstellzeit von 30 Minuten wurde eingehalten. Trotzdem könnte es während der Abformung zu Ungenauigkeiten wie zum Beispiel Verzerrungen gekommen sein. Auch eine Dimensionsänderung des Gipses würde die unterschiedliche Passung auf Masterstumpf und Meistermodell erklären. Ein falsches Anmischverhältnis von Wasser und Pulver zum Beispiel kann zu einer zu großen Abbindeexpansion führen (Marxkors et al. 2008). Den Herstellerangaben von $100 \mathrm{~g}$ Pulver zu $20 \mathrm{ml}$ destilliertem Wasser wurde allerdings Folge geleistet und mehrere Abformungen wurden mit demselben Mischungsverhältnis hintereinander ausgegossen. Dies macht Ungenauigkeiten durch fehlerhafte Anmischverhältnisse unwahrscheinlich. 
Da alle Käppchen der Testreihen 4, 11, 16 und 19 weder auf das Meister- noch auf das Mastermodell passten, sind in diesen Testreihen die Abweichungen nach der Herstellung des Meistermodells zu suchen. Es könnte zum Beispiel zu Ungenauigkeiten während der Digitalisierung oder bei der Bestimmung der Einschubrichtung gekommen sein.

Gegen einen Mangel der Fräsbahnen oder der Werkzeuge spricht, dass die Fräsbahnen für jedes Käppchen und Material der Testreihen einzeln bestimmt und für jedes Material geeignete Werkzeuge eingesetzt wurden.

In den Testreihen 3, 13 und 14 fiel auf, dass trotz desselben Innendesigns die metallischen Kronengerüste eine gute Passung hatten, während die keramischen Käppchen sich nicht in Sollposition bringen ließen. Dabei könnte das Werkzeug eine Rolle spielen, da es einen Unterschied in der Bearbeitung der Materialien gab. Die Metalle und Zirkoniumdioxid wurden gefräst, während die Glaskeramik-Käppchen mit Schleifern bearbeitet wurden.

Durch die unterschiedliche Beschaffenheit von Fräsen und Schleifern kommt es zu einer unterschiedlichen Abnutzung der Werkzeuge. Die einzelnen Werkzeuge wurden jedoch während der Studie nicht ausgetauscht, sodass alle Käppchen eines Materials mit denselben Werkzeugen bearbeitet wurden. Die Abnutzung der Werkzeuge kann auch als Grund für die mangelhaften Passungen der Keramiken ausgeschlossen werden, da die Käppchen der Testreihen 3, 13 und 14 nicht nacheinander gefertigt wurden. Auch der Unterschied zwischen den Abtragverfahren Schleifen und Fräsen kann für die schlechtere Passung der Keramiken nicht verantwortlich sein, da Glaskeramik geschliffen und Zirkoniumdioxid wie die metallischen Werksstoffe gefräst wurde.

Trotz Befolgung der Herstellerangaben können Ungenauigkeiten während des Sinterprozesses nicht ausgeschlossen werden. Es wurden allerdings mehrere Käppchen gleichzeitig gesintert und die Passungen der Keramikkäppchen von Testreihen aus dem gleichen Sintervorgang waren angemessen.

Testreihe Nummer 20 musste ebenfalls ausgeschlossen werden, da es hier bei der Bearbeitung des Glaskeramikrohlings zu einer Fraktur des Rohlings gekommen war. 
Die Käppchen der Testreihe Nummer 5 ließen sich ohne Probleme in Sollposition bringen, daher wurden diese Käppchen vermessen. Die Messungen zeigten jedoch, dass bei jedem Material die Käppchen der Testreihe Nummer 5 die schlechtesten mittleren Passungen aufwiesen. Auch die Maximalwerte wurden jeweils in dieser Testreihe gemessen.

Bei den Titan- und Kobalt-Chrom-Käppchen dieser Testreihe fiel auf, dass die Randspaltweiten sehr inhomogen waren. An benachbarten Messpunkten entstanden daher Unterschiede der Randspaltweite von über $120 \mu \mathrm{m}$.

Dieser inhomogene Randspalt könnte durch eine fehlerhafte Festlegung der Präparationsgrenze oder durch Ungenauigkeiten während der Digitalisierung enstanden sein.

Die makroskopische Passung war jedoch in Ordnung und mit bloßem Auge war kein deutlicher Randspalt zu erkennen. Da im klinischen Alltag die Passung auch oft ohne Zuhilfenahme von Lupenbrillen oder Mikroskopen beurteilt wird, werden solche Käppchen bei den Patienten eingesetzt. Daher wurden die Käppchen der Testreihe 5 trotz der signifikant schlechteren Werte nicht aus der Studie ausgeschlossen.

Um in weiterführenden Studien einige der beschriebenen Fehlerquellen zu umgehen, könnten auf die Abformungen und das Ausgießen der Abformungen verzichtet werden. Es könnte direckt der Masterstumpf eingescannt werden. Auf diese Weise würden auch die Passungen sich verbessern, da die Ungenauigkeiten der Abformung und der Gipsexpansion entfielen. Würde der Masterstumpf jedoch nicht mit einem Intraoralscanner sondern mit einem Labside-Scanner digitalisiert, wäre die Übertragbarkeit auf die klinische Situation stark eingeschränkt. Ob Intraoralscanner vergleichbare Passungen erzeugen, wie die Labside-Systeme müssen ebenfalls weiterführende Studien zeigen.

Zur Messung sollten die Käppchen nicht auf den Masterstumpf zementiert werden, da die Zementierung zu einer Vergrößerung des Randspalts führt (Borges et al. 2012; Demmel 1971; Stappert et al. 2001). Stattdessen wurde das Mastermodell in ein Gerät eingespannt, welches über eine Fixationsapparatur verfügt (siehe Abb. 14). Alle Käppchen wurden daher mit demselben okklusalen Druck auf dem Masterstumpf befestigt. 
Die Messungen wurden an digitalfotografisch erfassten Bildern durchgeführt. Diese Bilder wurden mit 35facher Vergrößerung unter einem Leica EZ4 D Auflichtmikroskop (Leica-Microsystems, Wetzlar) mit der integrierten Kamera erstellt. Um den Randspalt im $90^{\circ}$-Winkel zu fotografieren, konnte durch eine Rotationsachse der Fixationsapparatur der Kamera-Randspalt-Winkel variiert werden (siehe Abb. 13).

Die Messungen wurden mit dem Messwerkzeug der Adobe Photoshop CS5 Software (Adobe Systems Incorporated, San José, USA) durchgeführt.

Um den Randspalt vermessen zu können, musste die Mikroskop-Kamera-ComputerEinheit kalibriert werden. Zu diesem Zweck wurden Aufnahmen von Messkörpern (Motic Calibration Slide, Motic Deutschland $\mathrm{GmbH}$, Wetzlar) bekannter Größen erstellt und das Messwerkzeug geeicht. Diese Aufnahmen wurden zur späteren Reproduzierbarkeit archiviert.

Durch die Art der Aufnahmen konnten die Messungen im rechten Winkel zur Präparationsgrenze durchgeführt werden.

Die Ergebnisse zeigen, in welchem Ausmaß sich die Größe der Randspalten von Kronengerüsten aus verschiedenen Materialien unterscheiden, obwohl sie mit demselben CAD/CAM-System gefertigt wurden.

Die Mittelwerte der einzelnen Käppchen aus den unterschiedlichen Materialien liegen zwischen 31,11 $\mu \mathrm{m}$ und 97,20 $\mu \mathrm{m}$ und damit innerhalb des von McLean und von Fraunhofer (1971) postulierten klinischen Toleranzbereichs von bis zu $120 \mu \mathrm{m}$.

Trotzdem können Unterschiede in der Passgenauigkeit zwischen den verschiedenen Materialien festgestellt werden. Die Kronengerüste aus Titan und Kobalt-Chrom haben mit im Mittel 46,92 $\mu \mathrm{m}$ bzw. 48,37 $\mu \mathrm{m}$ die besten Passungen und unterscheiden sich nicht signifikant voneinander. Der Randspalt der Käppchen aus Zirkoniumdioxid ist jedoch im Schnitt ca. $20 \mu \mathrm{m}$ größer als der der Metallkäppchen, was zu einer signifikant schlechteren Passung führt.

Ein weiterer signifikanter Unterschied ergibt sich zwischen den Passungen der Titanund der Glaskeramik-Käppchen. Der Vergleich der Randspalten der Keramiken untereinander und der Vergleich zwischen Kobalt-Chrom und Glaskeramik führt zu keinen signifikanten Differenzen. 
In der Literatur wird vornehmlich die mittlere Passung der Restaurationen angegeben. Klinisch bedeutsamer sind allerdings die Maximalwerte der Randspalten der einzelnen Käppchen. Randspalten können sehr inhomogen verlaufen wie zum Beispiel in dieser Studie bei den Käppchen der Testreihe Nummer 5. Daher können auch Restaurationen mit einer sehr guten mittleren Passung an einigen Stellen Randspalten haben, die über dem klinischen Toleranzbereich liegen und damit den klinischen Langzeiterfolg determinieren.

Aus diesem Grund wurden in dieser Studie auch die maximalen Randspalten der Käppchen aus den verschiedenen Materialien verglichen. Hierzu wurden die jeweiligen Maximalwerte der einzelnen Käppchen desselben Materials gemittelt.

Dabei fiel auf, dass Titan mit 91,53 $\mu \mathrm{m}$ wieder den niedrigsten Wert hatte, KobaltChrom und Glaskeramik mit 96,86 $\mu \mathrm{m}$ bzw. 100,22 $\mu \mathrm{m}$ in der Mitte lagen und Zirkoniumdioxid mit 120,66 $\mu \mathrm{m}$ den größten Maximalwert besaß. Diese Unterschiede waren allerdings nicht signifikant.

Insgesamt lässt sich feststellen, dass die metallischen Werkstoffe in dieser Studie eine bessere Passung der Kronengerüste liefern als die Keramiken. Da es einen Unterschied in der Bearbeitung der Materialien gab, könnten die unterschiedlichen Werkzeuge von Bedeutung sein.

Die Glaskeramik-Käppchen wurden geschliffen, während die Metall- und Zirkoniumdioxid-Gerüste gefräst wurden.

Fräswerkzeuge werden hergestellt, indem das Werkzeug durch Abtrag von Material in eine bestimmte Form gebracht wird. Diese Form ermöglicht es dem Fräser, schneidend zu arbeiten, sodass Späne produziert werden. Da für diese Abtragmethode Kraft benötigt wird, kann sie für Glaskeramik nicht angewendet werden, da hierdurch Risse in der Glaskeramik entstehen könnten (Jorio et al. 2012). Daher wurden in dieser Arbeit für die Bearbeitung von Glaskeramik Diamantschleifer verwendet. Diamantschleifstifte werden durch Auftragen einer Verbundschicht mit eingefügten, scharfkantigen Diamantkörnern hergestellt. Schleifstifte tragen Material mit geringem Druck reibend ab, sodass ein pulverförmiger Abtrag entsteht.

Durch die verschiedenartige Beschaffenheit der Werkzeuge kommt es zu einem unterschiedlichen Verschleiß an diesen. 
Der Verschleiß von Fräsen kommt durch mehrere Mechanismen zustande. Verschleißursache können Adhäsion sowie Abrasion sein. Bei der Abrasion kommt es durch Reibung zu Materialabtrag am Werkzeug, während sich bei der Adhäsion ein Belag durch Wiederaufschweißen von Partikeln bildet. Darüber hinaus kann es auch zu Verformungen oder Brüchen der Schneidkanten kommen.

Bei Diamantschleifern entsteht der Verschleiß durch Kraft auf die einzelnen Diamantkörner. Je stumpfer die Körner sind, desto höher wird die auf sie einwirkende Kraft. Die Körner splittern scharfkantig ab oder sie brechen vollständig aus der Verbundschicht heraus und legen neue schneidende Diamanten frei. Diesen Prozess bezeichnet man als Selbstschärfung. Durch die herausbrechenden Körner verringert sich der Durchmesser der Werkzeuge in geringem Maße und die Größe der Körnung nimmt ab. Daher haben die Werkstücke, die mit einem abgenutzten Schleifwerkzeug bearbeitet werden, eine andere Oberflächenbeschaffenheit.

Die unterschiedlichen Abnutzungserscheinungen könnten Auswirkungen auf die Passungen der Käppchen haben. Daher wurden zur Bearbeitung der jeweiligen Materialien neue Werkzeuge eingesetzt. Die Stückzahlen waren gering, sodass Abnutzungserscheinungen in dieser Studie zu vernachlässigen sind. Gegen einen Effekt durch den Verschleiß der Werkzeuge spricht ebenfalls, dass das GlaskeramikKäppchen, welches als letztes bearbeitet wurde, die beste mittlere Passung aufwies.

Durch die verschiedenen Materialien und die unterschiedlichen Bearbeitungsweisen mussten voneinander abweichende Anpressdrücke während der Bearbeitung verwendet werden. Dies könnte zu unterschiedlichen Passungen geführt haben. Die Anpressdrücke waren jedoch auf die jeweiligen Material-Werkzeug-Paarungen abgestimmt und durch die Prozessbeschreibungen der Firma Wissner vorgegeben.

Die Parameter für das Innendesign der Käppchen wurden in der Pilotstudie an Kobalt-Chrom-Käppchen getestet. Nach der Pilotstudie wurden diese Parameter für alle weiteren Materialien übernommen. Aufgrund der Pilotstudie wurde der Parameter Zementspalt auf $30 \mu \mathrm{m}$ und der Abstand des Zementspalts von der Präparationsgrenze auf $1,5 \mathrm{~mm}$ festgelegt. 
Dies könnte ebenfalls ein Grund für die schlechtere Passung der Keramik-Käppchen im Vergleich zu den Metall-Käppchen sein. Kronen aus Keramik könnten einen größeren Zementspalt benötigen als Metallrestaurationen. Diese Vermutung wird von Hmaidouch et al. (2011) unterstützt. Sie fanden heraus, dass CAD/CAM-gefertigte Keramikkronen bei einem Zementspalt von $100 \mu \mathrm{m}$ eine bessere Passung haben als bei einem Zementspalt von $50 \mu \mathrm{m}$.

Im Gegensatz dazu vertreten Pospiech et al. (2002) die Ansicht, dass bei der Verwendung von Zirkoniumdioxid die besten Ergebnisse mit den in dieser Studie angewandten Parametern erzielt werden können.

Die schlechteren Passungen von Glaskeramik und Zirkoniumdioxid könnten auch an der verlängerten Prozesskette der Keramiken liegen.

Titan und Kobalt-Chrom brauchten nach dem Fräsen nicht weiter bearbeitet werden. Durch das ähnliche Herstellungsverfahren besitzen die Metall-Gerüste vergleichbare Randspaltwerte.

Zirkoniumdioxid und Glaskeramik mussten nach der CAM-Bearbeitung gesintert werden. Die Glaskeramik-Käppchen wurden in Originalgröße ausgeschliffen. Eventuelle Fehler könnten durch mögliche minimale Maßabweichungen während des Sinterns aufgetreten sein.

Zirkoniumdioxid wurde im Weißzustand gefräst. Da nach der Bearbeitung noch eine Sinterschwindung von $25 \%$ auftritt, wurden die Zirkoniumdioxid-Gerüste $25 \%$ vergrößert gefräst. Die Größenveränderung während des Sinterns könnte zu stärkeren Ungenauigkeiten geführt haben, als es bei den Glaskeramik-Käppchen der Fall war. Dies würde die bessere Passung der Glaskeramik-Käppchen im Vergleich zu den Zirkoniumdioxid-Gerüsten erklären. 
4.2 Vergleich der Ergebnisse der Studie mit anderen Studien

\begin{tabular}{|l|l|l|l|l|}
\hline & Titan & Kobalt-Chrom & Zirkonimdioxid & Glaskeramik \\
\hline Vorliegende Arbeit & 46,92 & 48,37 & 68,25 & 58,73 \\
\hline Besimo (1997) & 47 & & & \\
Halder (2003) & 45 & & & \\
\hline Han et al. (2011) & 51 & & & \\
\hline Karlsson (1993) & 60 & & & \\
Tan et al. (2008) & 79,43 & & & \\
Keul et al. (2014) & & $56,90 / 90,64$ & $127,23 / 141,08$ & \\
Örtorp et al. (2011) & & 166 & & \\
Tamac et al. (2014) & & 68,64 & & \\
Biscaro et al. (2013) & & & $34,18 / 35,32$ & \\
Euán et al. (2014) & & & $52,66 / 64,06$ & \\
Fornefett (2010) & & & $54,59-71,71$ & \\
Park et al. (2013) & & & 102,73 & \\
Srikakula et al. (2014) & & & $36,05 / 47,80 /$ & \\
Bindl und Mörmann (2007) & & & $55,79 / 64,08$ & \\
Keshvad et al. (2011) & & & & $<80$ \\
\hline Neves et al (2014) & & & & 36 (Inlay) \\
Vanlioglu et al. (2012) & & & & 112,14 (Onlay) \\
\hline
\end{tabular}

Tab. 5: Randspaltweite $[\mu \mathrm{m}]$ von Restaurationen aus unterschiedlichen Materialien in verschiedenen Studien

Mit einem Mittelwert von 46,92 $\mu \mathrm{m}$ wies Titan in der vorliegenden Studie den besten Randschluss auf. Besimo et al. (1997) und Halder (2003) erzielten an CAD/CAMgefertigten Titan-Käppchen ähnliche Ergebnisse. Halder ermittelte den Wert von $45 \mu \mathrm{m}$ allerdings erst nach Aufpassen der Käppchen. In der vorliegenden Studie wurden die Käppchen nicht manuell aufgepasst, da das Aufpassen eine signifikante Verkleinerung des Randspalts bewirkt (Fick 2003; Fornefett 2010; Witkowski et al. 2006) und die manuelle Veränderung nicht objektiv quantifizierbar ist. Die CAD/CAM-gefertigten Titan-Restaurationen von Tan et al. (2008) wiesen mit $79,43 \mu \mathrm{m}$ im Vergleich mit den anderen Studien einen relativ großen Randspalt auf (siehe Tab. 5). 
Die Testkäppchen dieser Studie aus Kobalt-Chrom erreichten mit 48,37 $\mu \mathrm{m}$ einen ähnlichen Randschluss wie die aus Titan.

Örtorp et al. (2011) verglichen unterschiedliche Herstellungsmethoden für festsitzenden Zahnersatz aus Kobalt-Chrom. Die CAD/CAM-gefertigten Restaurationen zeigten hierbei den schlechtesten Randschluss. Allerdings waren die Werte des mittels CAD/CAM-Technik hergestellten Zahnersatzes mit $166 \mu \mathrm{m}$ mehr als dreimal so hoch wie die Werte in der vorliegenden Arbeit.

In der Untersuchung von Tamac et al. (2014) wurden drei dieser Herstellungsverfahren erneut getestet. Es ergab sich für den marginalen Randspalt kein signifikanter Unterschied zwischen CAD/CAM-gefertigten, konventionell gegossenen und laser-gesinterten Kobalt-Chrom-Kronen. Der Randspalt der mittels eines CAD/CAM-Systems hergestellten Kronen betrug 86,64 $\mu \mathrm{m}$. Der kleinere Randspalt der CAD/CAM-Restaurationen von Tamac et al. könnte durch eine neuere Software und verbesserte Werkzeuge entstanden sein.

Die vermessenen Randspalten der Kobalt-Chrom-Käppchen in der Studie von Keul et al. (2014) betrugen abhängig vom Digitalisierungssystem Werte zwischen $56,90 \mu \mathrm{m}$ und $90,64 \mu \mathrm{m}$, was den Werten in der vorliegenden Studie nahezu entspricht.

Die ebenfalls von Keul et al. untersuchten Zirkoniumdioxid-Restaurationen lagen allerdings mit Randspaltwerten von 127,23 $\mu \mathrm{m}$ und $141,08 \mu \mathrm{m}$ oberhalb der klinischen Toleranzgrenze. Die vorliegende Studie ermittelte für Käppchen aus Zirkoniumdioxid den durchschnittlichen Wert von 68,25 $\mu \mathrm{m}$.

Dennoch gibt es Studien, in denen der Randspalt von CAD/CAM-gefertigten Zirkoniumdioxid-Kronen mit 34,18 $\mu \mathrm{m}$ die gleiche Größe aufwies wie der Randspalt von konventionell hergestellten, verblendeten Metallkronen (Biscaro et al. 2013).

Für die Restaurationen aus Glaskeramik wurden in der vorliegenden Studie Randspaltwerte von durchschnittlich 58,73 $\mu \mathrm{m}$ ermittelt.

Zu den Randspalten von CAD/CAM-gefertigten Glaskeramikkronen gibt es nur sehr wenige Untersuchungen. Eine davon ist die Studie von Bindl und Mörmann (2007) in der die Kronen Randspalten von ca. 80 mm aufwiesen. Neves et al. (2014) nutzten zur Digitalisierung der Stümpfe zwei verschiedene Chairside-Verfahren und erreichten mit dem CEREC-System sehr gute Randspaltwerte von 39,2 $\mu \mathrm{m}$. 
Vanlioglu et al. (2012) und Keshvad et al. (2011) untersuchten Glaskeramik-Onlays beziehungsweise Inlays. Die gemessenen Randspalten lagen bei $112,14 \mu \mathrm{m}$ (Onlays) und $36 \mu \mathrm{m}$ (Inlays).

Der in der Tabelle 5 dargestellte Vergleich ist nur eingeschränkt aussagekräftig, da in den Studien einige Ausgangsparameter wie zum Beispiel Präparationsdesign, Restaurationsart, Digitalisierungsverfahren und CAD/CAM-Systeme verschieden waren.

Besimo et al. (1997) beispielsweise verwendeten ein mechanisches Verfahren zur Digitalisierung der Stümpfe. In der Studie von Tan et al. (2008) wurde mit einem Konvergenzwinkel von $12^{\circ}$ und einer Hohlkehle ein Präparationsdesign gewählt, welches von dem in dieser Arbeit verwendeten Präparationsdesign stark abweicht.

Die untersuchten Restaurationen von Keul et al. (2014) wurden wie in der vorliegenden Studie mit einem CAM-System der Firma Wissner (Göttingen) hergestellt. Allerdings wurde nicht der Randschluss von Kronenkäppchen untersucht, sondern die marginale Passung von viergliedrigen Brückengerüsten. Weitspannige Brückengerüste aus Zirkoniumdioxid weisen eine schlechtere Passung auf als Einzelkronen der gleichen Herstellungsart (Sachs et al. 2014)

Biscaro et al. (2013) nutzten die in-vivo-Methode. Die extraktionswürdigen Zähne wurden am Patienten präpariert und abgeformt. Die Restaurationen wurden zementiert, bevor die Zähne extrahiert und vermessen wurden.

In der vorliegenden Studie wurden derselbe Masterstumpf und ein identischer Versuchsaufbau verwendet wie in der Studie von Fornefett (2010). Daher können die Randspalten der Zirkoniumdioxid-Restaurationen beider Studien besser miteinander verglichen werden als mit den Ergebnissen anderer Studien.

Fornefett verwendete lediglich andere CAD/CAM-Systeme und andere Scanner. Die dabei erzielten Werte von 54,59 bis $71,71 \mu \mathrm{m}$ sind mit den Randspalten der vorliegenden Studie für Käppchen aus Zirkoniumdioxid vergleichbar. 
Das in dieser Studie verwendete CAD/CAM-System (GAMMA 202 der Firma Wissner, Göttingen) ist unabhängig vom Material in der Lage, Einzel-kronengerüste mit klinisch akzeptabler marginaler Passung zu fertigen. Inwieweit dies mit anderen CAD/CAM-Systemen möglich ist, müssen weitere Studien klären.

\subsection{Schlussfolgerung für die Praxis}

Titan hatte in der vorliegenden Studie die geringsten marginalen Randspalten, während die Passung der Zirkoniumdioxid-Käppchen signifikant schlechter war als die Passung der Metall-Restaurationen.

Die mittleren Passungen aller Kronengerüste lagen unter der von McLean und von Fraunhofer (1971) angegebenen klinischen Toleranzgrenze von $120 \mu \mathrm{m}$ und auch die gemittelten Maximalwerte der Käppchen waren klinisch akzeptabel.

Titan hat durch seine Oxidschicht eine sehr gute Biokompatibilität (Kappert 1994) und auch die von Batson et al. (2014) getesteten Restaurationen aus Kobalt-Chrom, Zirkoniumdioxid und Glaskeramik riefen keine entzündlichen Reaktionen der Gingiva hervor, sodass auch unter diesem Aspekt alle hier verwendeten Materialien für den klinischen Einsatz zu empfehlen sind.

Dennoch können Titan und Kobalt-Chrom trotz der geringen marginalen Randspalten im hochästhetischen Bereich nur mit Einschränkung verwendet werden.

Daher muss für jeden Patienten individuell entschieden werden, welches Restaurationsmaterial eingesetzt werden soll. 


\section{Zusammenfassung}

In der vorliegenden Arbeit wurde die marginale Passgenauigkeit von CAD/CAMgefertigten Kronengerüsten aus verschiedenen dentalen Materialien untersucht.

Es wurde der Randspalt von Titan-, Kobalt-Chrom-, Zirkoniumdioxid- und Glaskeramik-Käppchen ermittelt. Ziel dieser Studie war es, Passungen von Kronenkäppchen aus verschiedenen Materialien zu vergleichen, wenn sie mit demselben CAD/CAM-System und dem gleichen Innendesign (=identische Prozesskette) gefertigt wurden. Gleichzeitig wurde überprüft, ob der Randschluss der Käppchen im klinisch akzeptablen Bereich von unter $120 \mu \mathrm{m}$ liegt.

Zur Vermessung des Randspalts wurden an dem Masterstumpf 24 Stellen markiert. Von jedem Messpunkt wurden unter 35facher Vergrößerung mit einer in einem Auflichtmikroskop integrierten Kamera Aufnahmen von jedem Käppchen angefertigt. Nach der Kalibrierung der Mikroskop-Kamera-Computer-Einheit wurde anhand der Fotos der Randspalt zwischen den Gerüsten und dem Stumpf an den Messpunkten ermittelt.

Es ergaben sich für die verschiedenen Materialien unterschiedliche Randspaltgrößen.

Titan hatte mit 46,92 $\mu \mathrm{m}$ einen signifikant geringeren mittleren Randspalt als Glaskeramik $(58,73 \mu \mathrm{m})$ und Zirkoniumdioxid $(68,25 \mu \mathrm{m})$. Ein weiterer signifikanter Unterschied ergab sich zwischen den gemittelten Passungen von Kobalt-Chrom $(48,37 \mu \mathrm{m})$ und Zirkoniumdioxid $(68,25 \mu \mathrm{m})$.

Bei dem Vergleich der gemittelten Maximalwerte der Materialien fiel auf, dass Titan mit 91,53 $\mu \mathrm{m}$ die niedrigsten Maxima aufwies. Für Kobalt-Chrom und Glaskeramik wurden Werte von 96,86 $\mu \mathrm{m}$ bzw. 100,22 $\mu \mathrm{m}$ ermittelt, und Zirkoniumdioxid besaß mit 120,66 $\mu \mathrm{m}$ die größten Maximalwerte. Diese Unterschiede waren allerdings nicht signifikant.

Die Mittelwerte aller Randspalten lagen innerhalb des postulierten klinischen Toleranzbereichs von $120 \mu \mathrm{m}$ und auch die Maximalwerte überstiegen die Toleranzgrenze in einem Fall nur geringfügig. Daher erschienen alle hier untersuchten Materialien für den klinischen Einsatz geeignet. 


\section{Literaturverzeichnis}

Aggstaller H, Beuer F, Funk M, Erdelt KJ, Gernet W (2006): Einfluss der Präparationsgeometrie auf die Bruchfestigkeit von Kronenkappen aus Zirkoniumdioxid.

Dtsch Zahnärztl Z $\underline{61}, 347-352$

An S, Kim S, Choi H, Lee JH, Moon HS (2014): Evaluating the marginal fit of zirconia copings with digital impressions with an intraoral digital scanner.

J Prosthet Dent 112, 1171-1175

Ariely E (1979): Vergleichende Untersuchungen der Beziehung zwischen Kronenrand und freier Gingiva.

Dtsch Zahnärztl Z $\underline{34}, 206$

Bader JD, Rozier RG, McFall WT Jr, Ramsey DL (1991): Effect of crown margins on periodontal conditions in regularly attending patients.

J Prosthet Dent $\underline{65}, 75-79$

Batson ER, Cooper LF, Duqum I, Mendonça G (2014): Clinical outcomes of three different crown systems with CAD/CAM technology.

J Prosthet Dent 112, 770-777

Belli R, Guimarães JC, Lohbauer U, Baratieri LN (2011): Über die Sprödigkeit von Dentalkeramiken - Die Ursachen für Frakturverluste.

Quintessenz $\underline{62}$, 49-61

Besimo C, Jeger C, Guggenheim R (1997): Marginal adaptation of titanium frameworks produced by CAD/CAM techniques.

Int J Prosthodont 10, 541-546 
Bindl A, Mörmann WH (2005): Marginal and internal fit of all-ceramic CAD/CAM crown-copings on chamfer preparations.

J Oral Rehabil $\underline{32}, 441-447$

Bindl A, Mörmann WH (2007): In-vitro-Untersuchung der Passgenauigkeit von vollkeramischen Seitenzahnbrücken-Gerüsten.

Parodontol Restaur Zahnheilkd 27, 538-547 (Abb. 2 Seite 543)

Biscaro L, Bonfiglioli R, Soattin M, Vigolo P (2013): An in vivo evaluation of fit of zirconium-oxide based ceramic single crowns, generated with two CAD/CAM systems, in comparison to metal ceramic single crowns.

J Prosthodont 22, 36-41

Borelli B, Sorrentino R, Goracci C, Zarone F, Ferrari M (2013): In vitro analysis of residual tooth structure of maxillary anterior teeth after different prosthetic finish line preparations for full-coverage single crowns.

J Oral Sci $\underline{55}, 79-84$

Borges GA, Faria JS, Agarwa PI, Spohr AM, Correr-Sobrinho L, Miranzi BAS (2012): In Vitro Marginal Fit of Three All-Ceramic Crown Systems Before and After Cementation.

Oper Dent $\underline{37}, 641-649$

Bürke H (2006): Zwei Glaskeramiken auf dem neuesten Stand IPS e.max Press und IPS e.max CAD.

Quintessenz Zahntech $\underline{\text { 32, }}$ 1316-1325

CAM-Fans aufgepasst: Maschinenvielfalt by WISSNER (2009): 0. Verf.;

DDN $\underline{3}$, 38-41 [http://www.ddn-online.net/artikel/item.php?itemid=453]

Cramer von Clausbruch S: Zirkon und Zirkonium, Steigerung oder unterschiedliche Materialien?; In: „dental-labor“ Fachbuchreihe, Band 2: Vollkeramik 2; Verlag Neuer Merkur GmbH, München 2004, 19-24 
Cramer von Clausbruch S (2005): Titanwerkstoffe - ein Vergleich.

Quintessenz Zahntech 31, 1208-1221

Czerny C (2003): Ein gutes Provisorium - Garant für prothetische Erfolge.

Quintessenz J $\underline{33}, 171-175$

Demmel HJ (1971): Der Einfluß verschiedener Zementsorten auf den Randschluß paßgenauer Kronen.

Dtsch Zahnärztl Z 트, 700-701

Edelhoff D, Beuer F, Schweiger J, Brix O, Stimmelmayr M, Güth JF (2012): CAD/CAM-generierte Restaurationen aus Hochleistungspolymer zur Vorbehandlung komplexer Fälle.

Quintessenz Zahntech $\underline{38}$, 794-805

Euán R, Figueras-Álvarez O, Cabratosa-Termes J, Oliver-Parra R (2014): Marginal adaptation of zirconium dioxide copings: Influence of the CAD/CAM system and the finish line design.

J Prosthet Dent 112, 155-162

Ewertz M (2013): In kurzer Zeit mit CAD/CAM zur Seitenzahnkrone.

Zahntech Wirtsch Labor 17, 33-37

Felton DA, Kanoy BE, Bayne SA, Wirthman GP (1991): Effect of in vivo crown margin discrepancies on periodontal health.

J Prosthet Dent $\underline{65}$, 357-364

Fick K: Marginaler Randspalt und innere Passung von CAM-gefrästen Kronengerüsten bei unterschiedlichem Präparationswinkel.

Med. Diss. München 2003

Fischer C (2008): Langzeitprovisorien für den Frontzahnbereich.

Quintessenz Zahntech $\underline{34}$, 676-681 
Fornefett D: Evaluation der marginalen Passgenauigkeit verschiedener CADScansysteme in Abhängigkeit vom okklusalen Öffnungswinkel der Präparation.

Med. Diss. Göttingen 2010

Fürst EM: Untersuchungen zur Passgenauigkeit von CAD/CAM-gefrästen ZirkonoxidEinzelkronengerüsten bei unterschiedlichem Präparationswinkel.

Med. Diss. München 2008

Funk M: In-vitro-Untersuchung zur Korrelation von Präparationsdesign und klinischer Haltbarkeit CAD/CAM-gefertigter Zirkondioxidkronen.

Med. Diss. München 2007

Geis-Gerstorfer J: Nichtedelmetalllegierungen; In: Zahnärztliche Werkstoffe und ihre Verarbeitung, Band 1: Grundlagen und Verarbeitung; hrsg. v. Eichner K, Kappert HF; Georg Thieme Verlag KG, Stuttgart 2005, 119-172

Gernet W, Mitteldorf J (1984): Untersuchungen über die Realisierbarkeit des supragingival gelegenen Kronenrandes.

Dtsch Zahnärztl Z $\underline{39}, 761-763$

Geurtsen W (1990): Der Kronen-und Füllungsrand.

Dtsch Zahnärztl Z $\underline{45}$, 380-386

von Gropp HP, Schwindling R (1971): Statistische Feststellungen über fehlerhafte Kronengestaltung mit Folgen auf Zahn, Parodont und Gingiva.

Dtsch Zahnärztl Z $\underline{26}, 734-742$

Günay H, Schulze A, Roßbach A, Geurtsen W (2001): Intrasulkuläre Zahnpräparation und parodontale Gesundheit - Eine prospektive klinische Studie.

Dtsch Zahnärztl Z $\underline{56}, 109-13$

Hajtó J (2010): Retentionen und Widerstandsform bei zementierten Kronen und Brücken.

Zahnheilkd Manage Kult 26 , 572-579 
Halder J: Marginale Passgenauigkeit CAD/CAM-gefertigter Titan-Kronenkäppchen. Med. Diss. München 2003

Hamza TA, Ezzat HA, El-Hossary MMK, El Megid Katamish HA, Shokry TE, Rosenstiel SF (2013): Accuracy of ceramic restorations made with two CAD/CAM systems.

J Prosthet Dent 109, 83-87

Han HS, Yang HS, Lim HP, Park YJ (2011): Marginal accuracy and internal fit of machine-milled and cast titanium crowns.

J Prosthet Dent 106, 191-197

Helkimo E, Carlsson GE, Helkimo M (1977): Bite force and state of dentition. Acta Odontol Scand $\underline{35}$, 297-303

Hewlett ER, Orro ME, Clark GT (1992): Accuracy testing of three-dimensional digitizing systems.

Dent Mater $\underline{8}, 49-53$

High Speed Cutting made in Göttingen (2009): o. Verf.;

DDN $\underline{3}, 80-86$ [http://www.ddn-online.net/artikel/item.php?itemid=473]

Hmaidouch R, Neumann P, Mueller WD (2011): Influence of preparation form, luting space setting and cement type on the marginal and internal fit of CAD/CAM crown copings.

Int J Comput Dent 14, 219-226

Hohmann W (1984): Können Kobaltbasisgußwerkstoffe eine Alternative zu Nickelbasisgußwerkstoffen sein?

Dtsch Zahnärztl Z $\underline{39}$, 782-783

Holmes JR, Bayne SC, Holland GA, Sulik WD (1989): Considerations in measurement of marginal fit.

J Prosthet Dent $\underline{62}, 405-408$ 
von Hüttner G (1971): Nachuntersuchungen von Kronen und Brückenzahnersatz in bezug auf den Kronenrand und das marginale Parodontium.

Dtsch Zahnärztl Z $\underline{26}$, 724-729

Hummel S, Seger J (2005): Ein Werkstoff für das Pressen und Schleifen.

Quintessenz Zahntech 31, 746-752

Janda R (2007a): Vollkeramiken: Zusammensetzung, Eigenschaften, Anwendung, Wertung.

Quintessenz Zahntech $\underline{33}, 46-60$

Janda R (2007b): Welches Zirkoniumoxid darf's denn sein?

Quintessenz Zahntech 33, 1351-1352

Jorio C, Dietz S, Jobst T (2012): CAD/CAM-Fräser: das unbekannte Werkzeug? Quintessenz Zahntech $\underline{38}$, 1220-1230

Jung T, Quast U (1971): Das Gefüge am Kronenrand.

Dtsch Zahnärztl Z $\underline{26}, 785-792$

Kappert HF (1994): Titan als Werkstoff für die zahnärztliche Prothetik und Implantologie.

Dtsch Zahnärztl Z $\underline{49}, 573-83$

Karlsson S (1986): A clinical evaluation of fixed bridges, 10 years following insertion. J Oral Rehabil 13 , 423-432

Karlsson S (1993): The fit of Procera titanium crowns: an in vitro and clinical study. Acta Odontol Scand $\underline{51}, 129-134$

Kerschbaum Th, Porschen C (1998): Kronenrandschluß und -konturqualität in fünf Dentallaboratorien.

Dtsch Zahnärztl Z $\underline{53}, 620-623$ 
Kerschbaum Th, Seth M, Teeuwen U (1997): Verweildauer von kunststoff- und metallkeramisch verblendeten Kronen und Brücken.

Dtsch Zahnärztl Z $\underline{52}, 404-406$

Kerschbaum Th, Faber FJ, Noll FJ, Keiner M, Hürther W, Schumacher S, Keller E (2009): Komplikationen von Cercon-Restaurationen in den ersten fünf Jahren.

Dtsch Zahnärztl Z $\underline{64}, 81-89$

Keshvad A, Hooshmand T, Asefzadeh F, Khalilinejad F, Alihemmati M, Van Noort R (2011): Marginal Gap, Internal Fit, and Fracture Load of Leucite-Reinforced Ceramic Inlays Fabricated by CEREC inLab and Hot-Pressed Techniques.

J Prosthodont 20, 535-540

Keul C, Stawarczyk B, Erdelt KJ, Beuer F, Edelhoff D, Güth JF (2014): Fit of 4-unit FDPs made of zirconia and CoCr-alloy after chairside and labside digitalization - A laboratory study.

Dent Mater 30, 400-407

Kobes LWR, Kaiser L, Weriz K (1988): Untersuchungen zur Paßgenauigkeit von Modellgußarbeiten.

Dtsch Zahnärztl Z $\underline{43}$, 515-519

Kohorst P, Brinkmann H, Li J, Borchers L, Stiesch M (2009): Marginal accuracy of four-unit zirconia fixed dental prostheses fabricated using different computer-aided design/ computer-aided manufacturing systems.

Eur J Oral Sci 117, 319-325

Kokubo Y, Ohkubo C, Tsumita M, Miyashita A, Vult von Steyern P, Fukushima S (2005): Clinical marginal and internal gaps of Procera AllCeram crowns.

J Oral Rehabil 32, 526-530

Kraft E (1969): Die Gestaltung des Kronenrandes.

Dtsch Zahnärztl Z 노, 750-757 
Küpper H, Marx R (1993): Röntgentransparenz von Titan und Früherkennung von Sekundärkaries.

Dtsch Zahnärztl Z $\underline{48}, 174-176$

Lachmann K: In - vitro - Untersuchung zur Passgenauigkeit von Kronen, die mit verschiedenen CAD/CAM-Systemen gefertigt wurden.

Med. Diss. Berlin 2010

Lava $^{\text {TM }}$ Chairside Oral Scanner C.O.S.

Digitale Abformung - Präzision die begeistert.

(Produktbroschüre, o. Verf.) 3M ESPE AG, Seefeld 2011

[http://multimedia.3m.com/mws/media/7144450/lava-c-o-s-image-folder-

d.pdf?fn=Lava_COS_Image_F_D.pdf]

Lava $^{\text {TM }}$ Scan ST Design System

Dentales Scannen - vielseitig und präzise wie nie.

(Produktbroschüre, o. Verf.) 3M ESPE AG, Seefeld 2011

[http://multimedia.3m.com/mws/media/7492060/lava-scan-st-design-system-lava-designsoftware-7-sellsheet-d.pdf?fn=Lava_Design_ScanST_SW7_SS_D.pdf]

Lee KB, Park CW, Kim KH (2008): Marginal and internal fit of all-ceramic crowns fabricated with two different CAD/CAM systems.

Dent Mater J 27, 422-426

Lehmann KM, Hellwig E, Wenz HJ: Einführung in die Zahnheilkunde (Zahnärztliche Propädeutik). 11. Auflage; Deutscher Zahnärzte Verlag, Köln 2009

Lenz E: Dentallegierungen; In: Klinische Materialkunde für Zahnärzte; hrsg. v. Meiners H und Lehmann KM; Carl Hanser Verlag, München 1998, 71-118

Leong D, Chai J, Lautenschlager E, Gilbert J (1994): Marginal Fit of Machine-Milled Titanium and Cast Titanium Single Crowns.

Int J Prosthodont $\underline{7}, 440-447$ 
Luthardt RG, Sandkuhl O, Herold V, Walter MH (2001): Accuracy of mechanical digitizing with a CAD/CAM system for fixed restorations.

Int J Prosthodont 14, 146-151

Luthardt RG, Kordaß B, Moldovan O: Kronen und Brücken: CAD/CAM-Verfahren; In: Lehrbuch der zahnärztlichen Prothetik für Studium und Beruf; hrsg. v. Marxkors R;

Deutscher Zahnärzte Verlag, Köln 2009, 109-124

Marxkors R: Kronen; In: Lehrbuch der zahnärztlichen Prothetik für Studium und Beruf; hrsg. v. Marxkors R; Deutscher Zahnärzte Verlag, Köln 2009, 1-72

Marxkors R, Meiners H, Geis-Gerstorfer J: Taschenbuch der zahnärztlichen Werkstoffkunde

Vom Defekt zur Restauration. 6. Auflage; Deutscher Zahnärzte Verlag, Köln 2008

McLean JW, von Fraunhofer JA (1971): The estimation of cement film thickness by an in vivo technique.

Br Dent J 131, 107-111

Mehl A, Ender A, Mörmann W, Attin T (2009): Accuracy Testing of a New Intraoral 3D Camera.

Int J Comput Dent 12, 11-28

Meyer E, Eichner K (1980): Klinische Untersuchungsergebnisse zu verblendeten Kronen und Brücken (Vergleich Kunststoff/Keramik).

Dtsch Zahnärztl Z $\underline{35}, 864-869$

Müller M, Trützschler S (2012): Optische Abformung im Rahmen von Kronenversorgungen im Seitenzahngebiet - Erfahrungsbericht.

Dtsch Zahnärztl Z $\underline{67}, 164-168$ 
Neves FD, Prado CJ, Prudente MS, Carneiro TAPN, Zancopé K, Davi LR, Mendonça G., Cooper LF, Soares CJ (2014): Micro-computed tomography evaluation of marginal fit of lithium disilicate crowns fabricated by using chairside CAD/CAM systems or the heat-pressing technique.

J Prosthet Dent 112, 1134-1140

Noack T: In-vivo Abformgenauigkeit von A-Silikonen.

Med. Diss. Gießen 2012

Örtorp A, Jönsson D, Mouhsen A, Vult von Steyern P (2011): The fit of cobaltchromium three-unit fixed dental prostheses fabricated with four different techniques: A comparative in vitro study.

Dent Mater 27, 356-363

Park JH, Kwon TK, Yang JH, Han JS, Lee JB, Kim SH, Yeo IS (2013): A Comparative Study on the Marginal Fit of Zirconia Cores Manufactured by CAD/CAM and Copy Milling Methods.

Dentistry $\underline{3}, 163$ doi:10.4172/2161-1122.1000163

Pospiech P (2010): Chipping- systemimmanente oder verarbeitungsbedingte Probleme?

Quintessenz $\underline{61}, 173-181$

Pospiech P, Schweiger J, Meinen J (2002): Erste Erfahrungen mit dem neuen Vollkeramik-System Lava

Vom Zirkonoxidgerüst zur Lava-Vollkeramik.

Dent Labor (Munch) 무, 59-67

Rehberg HJ (1971): Exakter Randschluß - was ist das?

Dtsch Zahnärztl Z 26, 696-699 
Reich S, Gozdowski S, Trentzsch L, Frankenberger R, Lohbauer U (2008a): Marginal Fit of Heat-pressed vs CAD/CAM Processed All-ceramic Onlays Using a Milling Unit Prototype.

Oper Dent $\underline{33}, 644-650$

Reich S, Kappe K, Teschner H, Schmitt J (2008 b): Clinical fit of four-unit zirconia posterior fixed dental prostheses.

Eur J Oral Sci $\underline{116}, 579-584$

Reich S, Vollborn T, Wolfart S (2012): Die optische intraorale Abformung - vier Systeme im Überblick.

Dtsch Zahnärztl Z $\underline{67}, 177-189$

Reiss B (2006): Clinical results of Cerec inlays in a dental practice over a period of 18 years.

Int J Comput Dent $\underline{\underline{9}}, 11-22$

Reiss B (2007): Cerec Standard 3-D Okklusal Contouring in Comparision with the New Biogeneric Occlusal Morphing: A Case Report.

Int J Comput Dent $\underline{10}, 69-75$

Rinke S, Fischer C (2012): Indikationsbereiche für transluzente Zirkonoxidkeramikvarianten

Klinische und zahntechnische Aspekte.

Quintessenz $\underline{63}$, 895-905

Riquier R, Krause M (2011): Untersuchungsergebnisse zur Zerspanbarkeit handelsüblicher CoCr-Blanks.

Quintessenz Zahntech 37, 628-639

Rißmann J (2009): Kollegentipps

Mit der HSC-Fräsmaschine GAMMA 303 bin ich unabhängig.

Int Zahntech Mag 13, 769 - 771 
Reppel PD, Böning K, Walter M (1991): Festsitzender Zahnersatz aus gefrästem/funkenerodiertem Titan.

Dtsch Zahnärztl Z $\underline{46}, 756-758$

Rödiger M, Gersdorff N, Hüls A, Rinke S (2010): 4-Jahres-Ergebnisse zur klinischen Bewährung von konventionell zementierten Zirkonoxidbrücken im Seitenzahnbereich. Quintessenz $\underline{61}, 1213-1220$

Sachs C, Groesser J, Stadelmann M, Schweiger J, Erdelt K, Beuer F (2014): Fullarch prostheses from translucent zirconia: Accuracy of fit.

Dent Mater 30, 817-823

Schaudig A, Lehmann KM, Gente M (2009): Die Passgenauigkeit von Primärkronen in Abhängigkeit von der Legierung.

Quintessenz Zahntech $\underline{35}$, 58-63

von Schroeter P, Jürgensen B, Zöllner M (2004): Cercon move - A Navigation Aid for Dental CAD Applications.

Int J Comput Dent $\underline{7}, 371-377$

Schwickerath H (1979): Randspalt und Löslichkeit.

Dtsch Zahnärztl Z $\underline{34}$, 664-669

Spiekermann H (1986): Zur marginalen Paßform von Kronen und Brücken.

Dtsch Zahnärztl Z $\underline{41}, 1015-1019$

Srikakula NK, Babu CS, Reddy JRK, Saiprasad SH, Raju ASR (2014): Comparison of Marginal fit of Zirconium Oxide Copings Generated Using Four Different CAD-CAM Systems - An in Vitro Study.

J Res Adv Dent $\underline{3}, 163-171$

Stappert CFJ, Dai M, Heydecke G (2001): Randspaltanalyse dreigliedriger Voll- und Metallkeramikseitenzahnbrücken.

Dtsch Zahnärztl Z $\underline{56}$, 626-628 
Stawarczyk B, Trottmann A, Fischer J (2008): Bruchlast konventionell oder mittels $\mathrm{CAD} / \mathrm{CAM}$ hergestellter Brücken-Provisorien.

Quintessenz Zahntech 34, 412-421

Stawarczyk B, Ender A, Trottmann A, Hämmerle CHF (2010): Einfluss der Verbinderquerschnittfläche auf die Bruchlast von dreigliedrigen Provisorien. Konventionell versus CAD/CAM.

Quintessenz Zahntech 즈, 922-928

Tamac E, Toksavul S, Toman M (2014): Clinical marginal and internal adaptation of CAD/CAM milling, laser sintering, and cast metal ceramic crowns.

J Prosthet Dent 112, 909-913

Tan PL, Gratton DG, Diaz-Arnold AM, Holmes DC (2008): An in vitro comparison of vertical marginal gaps of CAD/CAM titanium and conventional cast restorations.

J Prosthodont 17, 378-383

Tinschert J, Natt G, Doose B, Fischer H, Marx R (1999): Seitenzahnbrücken aus hochfester Strukturkeramik.

Dtsch Zahnärztl Z $\underline{54}, 545-550$

Vanlioglu BA, Evren B, Yildiz C, Uludamar A, Kulak Özkan Y (2012): Internal and marginal adaptation of pressable and computer-aided design/computer-assisted manufacture onlay restorations.

Int J Prosthodont 25, 262-264

Vigolo P, Fonzi F (2008): An In Vitro Evaluation of Fit of Zirconium-Oxide-Based Ceramic Four-Unit Fixed Partial Dentures, Generated with Three Different CAD/CAM Systems, before and after Porcelain Firing Cycles and after Glaze Cycles.

J Prosthodont 17, 621-626 
Vigolo P, Mutinelli S (2012): Evaluation of zirconium-oxide-based ceramic single-unit posterior fixed dental prostheses (FDPs) generated with two CAD/CAM systems compared to porcelain-fused-to-metal single-unit posterior FDPs: a 5-year clinical prospective study.

J Prosthodont 21, 265-269

Wischropp T (2008): Zwei sind keines zu viel! Der Nutzen zweier CAD/CAM-Systeme in einem Labor.

Digit Dent News $\underline{2}, 50-58$

Wisithphrom K, Murray, PE, About I, Windsor LJ (2006): Wechselwirkungen zwischen Kavitätenpräparation und Restaurationsmaßnahmen und ihre Auswirkungen auf die Pulpavitalität.

Int J Parodontol Restaur Zahnheilkd 26, 588-597

Witkowski S, Komine F, Gerds T (2006): Marginal accuracy of titanium copings fabricated by casting and CAD/CAM techniques.

J Prosthet Dent $\underline{96}, 47-52$ 


\section{Danksagung}

Mein besonderer Dank gilt zunächst Herrn Priv.-Doz. Dr. med. dent. Matthias Rödiger für die Überlassung dieses interessanten Themas und für die kompetente Betreuung dieser Dissertation.

Herrn Priv.-Doz. Dr. med. dent. Nikolaus Gersdorff danke ich für seine vielfältige Hilfsbereitschaft.

Für die Unterstützung im Rahmen der statistischen Auswertung danke ich Herrn Thomas Asendorf.

Der Wissner $\mathrm{GmbH}$, insbesondere Herrn Dirk Lange, gebührt mein Dank für die Bereitstellung des CAD/CAM-Systems inklusive der Fräsmaschine GAMMA 202 und die fachliche Unterstützung.

Bei der Eisenbacher Dentalwaren ED GmbH und der METOXIT AG bedanke ich mich für die Bereitstellung der Kobalt-Chrom- und der Zirkoniumdioxid-Rohlinge. 


\section{Lebenslauf}

Am 29.08.1988 wurde ich als zweite Tochter von Prof. Dr.-Ing. Paul Gronau und Carola Gronau, geb. Michels, in Bergisch Gladbach geboren.

Von 1995 bis 1999 besuchte ich die Emhildis Grundschule in Meschede, von 1999 bis 2008 das städtische Gymnasium der Stadt Meschede. Dort erlangte ich im Mai 2008 die allgemeine Hochschulreife. Im Oktober 2008 begann ich das Studium der Zahnmedizin an der Georg-August-Universität Göttingen. Im August 2009 legte ich die naturwissenschaftliche Vorprüfung und im März 2011 die zahnärztliche Vorprüfung ab.

Das Studium der Zahnmedizin schloss ich im November 2013 mit dem Bestehen des Staatsexamens ab.

Im gleichen Monat begann ich meine Dissertation in der Poliklinik für Zahnärztliche Prothetik im Zentrum Zahn-, Mund- und Kieferheilkunde der Universitätsmedizin Göttingen unter der Betreuung von Herrn Priv.-Doz. Dr. med. dent. Matthias Rödiger. Seit Juni 2014 arbeite ich als Vorbereitungsassistentin. 